\title{
Enantioselective anti- and syn-(Borylmethyl)allylation of Aldehydes via Brønsted Acid Catalysis
}

\author{
Jiaming Liu and Ming Chen* \\ Department of Chemistry and Biochemistry
}

Auburn University

E-mail: mzc0102@auburn.edu

Supporting Information: Experimental Procedures, Tabulated Spectroscopic Data, ${ }^{1} \mathrm{H}$ and

${ }^{13} \mathrm{C}$ Spectra of New Compounds

SI-1 
General Experimental Details. All reaction solvents were purified before use. Tetrahydrofuran, diethyl ether and toluene were purified by passing through a solvent column composed of activated A-1 alumina. Unless indicated otherwise, all reactions were conducted under an atmosphere of argon using flame-dried or oven-dried $\left(120{ }^{\circ} \mathrm{C}\right)$ glassware. The term "concentrated under reduced pressure" refers to the removal of solvents and other volatile materials using a rotary evaporator with the water bath temperature below $30{ }^{\circ} \mathrm{C}$, followed by the removal of residual solvents at high vacuum $(<$ 0.2 mbar).

Proton nuclear magnetic resonance $\left({ }^{1} \mathrm{H}\right.$ NMR) spectra were acquired on commercial instruments $(400,500$ and $600 \mathrm{MHz})$ at Auburn University NMR facility. Carbon-13 nuclear magnetic resonance $\left({ }^{13} \mathrm{C}\right.$ NMR $)$ spectra were acquired at 101, 126 and $151 \mathrm{MHz}$. The proton signal for the residual non-deuterated solvent ( $\delta 7.26$ for chloroform) was used as an internal reference for ${ }^{1} \mathrm{H}$ NMR spectra. For ${ }^{13} \mathrm{C}$ NMR spectra, chemical shifts are reported relative to the resonance of chloroform $(\delta$ 77.36). Coupling constants are reported in Hz. High-resolution mass spectra were recorded on a commercial high-resolution mass spectrometer via the Micro Mass/Analytical Facility operated by the College of Chemistry and Biochemistry, Auburn University.

Analytical thin layer chromatography (TLC) was performed on Kieselgel 60 F254 glass plates precoated with a $0.25 \mathrm{~mm}$ thickness of silica gel. The TLC plates were visualized with UV light and/or by staining with Hanessian solution (ceric sulfate and ammonium molybdate in aqueous sulfuric acid) or $\mathrm{KMnO}_{4}$. Column chromatography was generally performed using Kieselgel 60 (230-400 mesh) silica gel, typically using a 50-100:1 weight ratio of silica gel to crude product. 


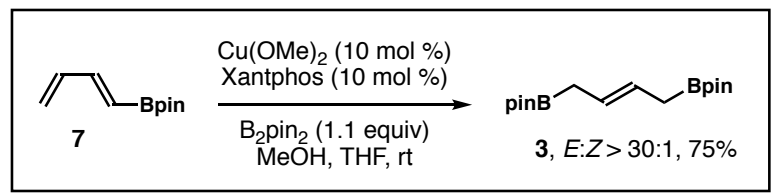

(E)-1,4-bis(4,4,5,5-tetramethyl-1,3,2-dioxaborolan-2-yl)but-2-ene (3): In an Ar-filled glove box, $\mathrm{Cu}(\mathrm{OMe})_{2}(10 \mathrm{mg}, 0.08 \mathrm{mmol}, 10 \mathrm{~mol} \%$ ), Xantphos (46 mg, $0.08 \mathrm{mmol}, 10$ mol \%), a Teflon-coated magnetic stir bar, and THF $(1.5 \mathrm{~mL})$ were sequentially added into a 1-dram vial. The mixture was stirred for $15 \mathrm{~min}$ at ambient temperature in the glove box. $\mathrm{B}_{2} \mathrm{Pin}_{2}(211 \mathrm{mg}, 0.83 \mathrm{mmol}, 1.1$ equiv) was added and the mixture was stirred for $5 \mathrm{~min}$. Then dienylboronate 7 (135 mg, $0.75 \mathrm{mmol}, 1.0$ equiv) and $\mathrm{MeOH}(30 \mu \mathrm{L}$, $0.75 \mathrm{mmol}, 1.0$ equiv) were added to the mixture sequentially and the resulting mixture was kept stirring at ambient temperature. After complete consumption of diene 7, $\mathrm{Et}_{2} \mathrm{O}(2$ $\mathrm{mL}$ ) was added to the vial, and the reaction mixture was filtered through a pad of Celite. The solution was concentrated under reduced pressure. Purification of the crude product was performed by flash chromatography (gradient elution with hexane and ethyl acetate, $30: 1$ to $10: 1)$ to give product 3 in $75 \%$ yield (173 $\mathrm{mg}, E: Z>30: 1)$ as a white solid. A 1 -mmol scale reaction was also conducted to give product 3 in $75 \%$ yield (231 mg, E:Z> 30:1). ${ }^{1} \mathrm{H}$ NMR (600 MHz, $\left.\mathrm{CDCl}_{3}\right) \delta 5.39-5.45$ (m, 2H), 1.64 (app. s, 4H), 1.23 (s, 24H). ${ }^{13} \mathrm{C} \mathrm{NMR}\left(151 \mathrm{MHz}, \mathrm{CDCl}_{3}\right) \delta 125.6,83.4,25.1,16.5$. HRMS $\left(\mathrm{ESI}^{+}\right): \mathrm{m} / z$ for $\mathrm{C}_{16} \mathrm{H}_{31} \mathrm{~B}_{2} \mathrm{O}_{4}[\mathrm{M}+\mathrm{H}]^{+}$calcd. 309.2413, found 309.2408.

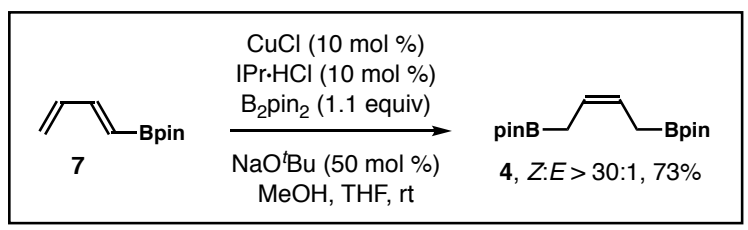

(Z)-1,4-bis(4,4,5,5-tetramethyl-1,3,2-dioxaborolan-2-yl)but-2-ene (4): In an Ar-filled glove box, $\mathrm{CuCl}$ (7 mg, $0.08 \mathrm{mmol}, 10 \mathrm{~mol} \%$ ), IPr $\mathrm{HCl}$ (32 mg, $0.08 \mathrm{mmol}, 10 \mathrm{~mol} \%$ ), $\mathrm{NaO}^{t} \mathrm{Bu}(37 \mathrm{mg}, 0.38 \mathrm{mmol}, 50 \mathrm{~mol} \%$ ), a Teflon-coated magnetic stir bar, and THF (1.5 $\mathrm{mL}$ ) were sequentially added to a 1-dram vial. The mixture was stirred for $15 \mathrm{~min}$ at ambient temperature in the glove box. $\mathrm{B}_{2} \mathrm{Pin}_{2}(211 \mathrm{mg}, 0.83 \mathrm{mmol}, 1.1$ equiv) was added and the mixture was stirred for $5 \mathrm{~min}$. Then dienylboronate 7 (135 $\mathrm{mg}, 0.75 \mathrm{mmol}, 1.0$ equiv) and $\mathrm{MeOH}(60 \mu \mathrm{L}, 1.5 \mathrm{mmol}, 2.0$ equiv) were added to the mixture sequentially and the resulting mixture was kept stirring at ambient temperature. After complete consumption of diene 7, $\mathrm{Et}_{2} \mathrm{O}(2 \mathrm{~mL})$ was added to the vial, and the reaction mixture was filtered through a pad of Celite. The solution was concentrated under reduced pressure. 
Purification of the crude product was performed by flash chromatography (gradient elution with hexane and ethyl acetate, 30:1 to 10:1) to give the product 4 in $73 \%$ yield (169 mg, Z:E > 30:1) as a white solid. A 1-mmol-scale reaction was also conducted to give product 4 in $83 \%$ yield $(256 \mathrm{mg}) .{ }^{1} \mathrm{H}$ NMR $\left(500 \mathrm{MHz}, \mathrm{CDCl}_{3}\right) \delta 5.47-5.54(\mathrm{~m}, 2 \mathrm{H})$, $1.65(\mathrm{~d}, J=4.6 \mathrm{~Hz}, 4 \mathrm{H}), 1.23(\mathrm{~s}, 24 \mathrm{H}) .{ }^{13} \mathrm{C}$ NMR $\left(126 \mathrm{MHz}, \mathrm{CDCl}_{3}\right) \delta 124.5,83.5,25.1$, 11.7. HRMS (ESI $\left.{ }^{+}\right): m / z$ for $\mathrm{C}_{16} \mathrm{H}_{31} \mathrm{~B}_{2} \mathrm{O}_{4}[\mathrm{M}+\mathrm{H}]^{+}$calcd. 309.2413, found 309.2408 .

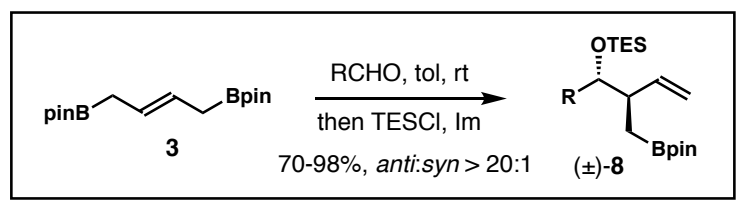

General procedure for the synthesis of TES-protected homoallylic alcohols ( \pm )-8: To a reaction vial containing a Teflon-coated magnetic stirring bar was added freshly distilled aldehyde $(0.1 \mathrm{mmol}, 1.0$ equiv, if it is a liquid). Toluene $(0.3 \mathrm{~mL})$ was added to the vial followed by addition of allylboronate $3(40 \mathrm{mg}, 0.13 \mathrm{mmol}, 1.3$ equiv). The reaction mixture was allowed to stir at ambient temperature for $12 \mathrm{~h}$. After complete consumption of the aldehyde, TESCl $(23 \mathrm{mg}, 0.15 \mathrm{mmol}, 1.5$ equiv), imidazole ( $14 \mathrm{mg}$, $0.2 \mathrm{mmol}, 2.0$ equiv) and DMF $(0.2 \mathrm{ml})$ were added to the vial. The reaction mixture was stirred at ambient temperature for additional $4 \mathrm{~h}$. Then brine $(1 \mathrm{~mL})$ and $\mathrm{Et}_{2} \mathrm{O}(0.5 \mathrm{~mL})$ were added to the reaction mixture. The organic layer was separated and the aqueous layer was extracted with $\mathrm{Et}_{2} \mathrm{O}(3 \times 1 \mathrm{~mL})$. The combined organic extracts were dried over anhydrous magnesium sulfate, filtered, and concentrated under reduced pressure. Purification of the crude product was performed by flash chromatography (gradient elution with hexane and ethyl acetate) to give product $( \pm)-\mathbf{8}$.

rac-Triethyl(((1R,2R)-1-phenyl-2-((4,4,5,5-tetramethyl-1,3,2-dioxaboro
lan-2-yl)methyl)but-3-en-1-yl)oxy)silane (8a) Prepared according to the
general procedure. The crude mixture was purified by column chromatography (hexane:ethyl acetate $=80: 1$ to $60: 1$ ) to give compound $\mathbf{8 a}$ in $85 \%$ yield $(34 \mathrm{mg})$ as colorless oil. ${ }^{1} \mathrm{H}$ NMR $\left(600 \mathrm{MHz}, \mathrm{CDCl}_{3}\right) \delta 7.24-7.26(\mathrm{~m}, 4 \mathrm{H}), 7.17-7.21$ (m, 1H), 5.80 (ddd, $J=17.7,10.3,8.1 \mathrm{~Hz}, 1 \mathrm{H}), 4.93(\mathrm{~d}, J=10.3 \mathrm{~Hz}, 1 \mathrm{H}), 4.89$ (d, $J=$ $17.3 \mathrm{~Hz}, 1 \mathrm{H}), 4.58(\mathrm{~d}, J=5.3 \mathrm{~Hz}, 1 \mathrm{H}), 2.52-2.57(\mathrm{~m}, 1 \mathrm{H}), 1.21$ (s, 6H), 1.19 (s, 6H), $0.84-0.90(\mathrm{~m}, 10 \mathrm{H}), 0.77(\mathrm{dd}, J=15.3,10.2 \mathrm{~Hz}, 1 \mathrm{H}), 0.44-0.53(\mathrm{~m}, 6 \mathrm{H}) .{ }^{13} \mathrm{C} \mathrm{NMR}$ $\left(151 \mathrm{MHz}, \mathrm{CDCl}_{3}\right) \delta 144.4,140.6,127.8,127.3,127.1,115.3,83.3,79.4,48.4,25.3,25.1$, 13.8, 7.2, 5.2. HRMS $\left(\mathrm{ESI}^{+}\right): \mathrm{m} / \mathrm{z}$ for $\mathrm{C}_{23} \mathrm{H}_{40} \mathrm{BO}_{3} \mathrm{Si}[\mathrm{M}+\mathrm{H}]^{+}$calcd. 403.2840 , found: 403.2825 . 


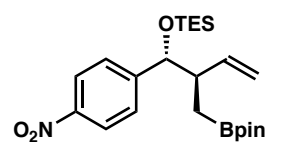

rac-Triethyl(((1R,2R)-1-(4-nitrophenyl)-2-((4,4,5,5-tetramethyl-1,3, 2-dioxaborolan-2-yl)methyl)but-3-en-1-yl)oxy)silane (8b) Prepared

according to the general procedure. The crude mixture was purified by column chromatography (hexane:ethyl acetate $=80: 1$ to $60: 1$ ) to give compound $\mathbf{8 b}$ in $83 \%$ yield $(37 \mathrm{mg})$ as colorless oil. ${ }^{1} \mathrm{H}$ NMR $\left(600 \mathrm{MHz}, \mathrm{CDCl}_{3}\right) \delta 8.13(\mathrm{~d}, J=8.5 \mathrm{~Hz}, 2 \mathrm{H})$, $7.42(\mathrm{~d}, J=8.4 \mathrm{~Hz}, 2 \mathrm{H}), 5.67-5.74(\mathrm{~m}, 1 \mathrm{H}), 4.93(\mathrm{~d}, J=10.2 \mathrm{~Hz}, 1 \mathrm{H}), 4.80$ (d, $J=17.2$ $\mathrm{Hz}, 1 \mathrm{H}), 4.74(\mathrm{~d}, J=4.2 \mathrm{~Hz}, 1 \mathrm{H}), 2.50-2.54(\mathrm{~m}, 1 \mathrm{H}), 1.22(\mathrm{~s}, 6 \mathrm{H}), 1.20(\mathrm{~s}, 6 \mathrm{H}), 0.95$ (dd, $J=15.4,4.9 \mathrm{~Hz}, 1 \mathrm{H}), 0.87(\mathrm{t}, J=7.9 \mathrm{~Hz}, 9 \mathrm{H}), 0.81$ (dd, $J=15.4,9.9 \mathrm{~Hz}, 1 \mathrm{H}), 0.38-$ $0.55(\mathrm{~m}, 6 \mathrm{H}) .{ }^{13} \mathrm{C}$ NMR $\left(151 \mathrm{MHz}, \mathrm{CDCl}_{3}\right) \delta 152.2,147.3,139.1,127.8,123.3,116.3$, 83.5, 78.4, 48.5, 25.3, 25.1, 13.8, 7.1, 5.2. HRMS $\left(\mathrm{ESI}^{+}\right)$: $\mathrm{m} / \mathrm{z}$ for $\mathrm{C}_{23} \mathrm{H}_{39} \mathrm{BO}_{5} \mathrm{NSi}[\mathrm{M}+\mathrm{H}]^{+}$ calcd. 448.2691, found: 448.2709 .

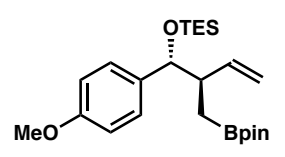

rac-Triethyl(((1R,2R)-1-(4-methoxyphenyl)-2-((4,4,5,5-tetramethyl1,3,2-dioxaborolan-2-yl)methyl)but-3-en-1-yl)oxy)silane

Prepared according to the general procedure. The crude mixture was purified by column chromatography (hexane:ethyl acetate $=80: 1$ to $60: 1$ ) to give compound $8 \mathbf{c}$ in $81 \%$ yield $(35 \mathrm{mg})$ as colorless oil. ${ }^{1} \mathrm{H}$ NMR $\left(600 \mathrm{MHz}, \mathrm{CDCl}_{3}\right) \delta 7.17$ (d, $J=8.5 \mathrm{~Hz}, 2 \mathrm{H}), 6.79$ (d, $J=8.5 \mathrm{~Hz}, 2 \mathrm{H}), 5.79$ (ddd, $J=17.7,10.4,8.1 \mathrm{~Hz}, 1 \mathrm{H}), 4.93$ (d, $J=10.4 \mathrm{~Hz}, 1 \mathrm{H}), 4.90(\mathrm{~d}, J=18.0 \mathrm{~Hz}, 1 \mathrm{H}), 4.51(\mathrm{~d}, J=5.5 \mathrm{~Hz}, 1 \mathrm{H}), 3.79(\mathrm{~s}, 3 \mathrm{H})$, $2.49-2.53$ (m, 1H), 1.20 (s, 6H), 1.19 (s, 6H), $0.78-0.87$ (m, 10H), 0.73 (dd, $J=15.2$, $10.3 \mathrm{~Hz}, 1 \mathrm{H}), 0.34-0.49$ (m, 6H). ${ }^{13} \mathrm{C}$ NMR $\left(151 \mathrm{MHz}, \mathrm{CDCl}_{3}\right) \delta 158.8,140.9,136.7$,

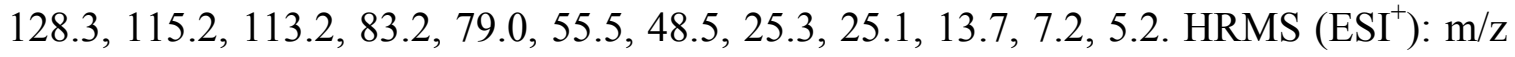
for $\mathrm{C}_{24} \mathrm{H}_{41} \mathrm{BO}_{4} \mathrm{NaSi}[\mathrm{M}+\mathrm{Na}]^{+}$calcd. 455.2765 , found: 455.2785 .

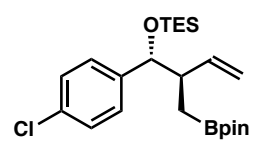

rac-(((1R,2R)-1-(4-chlorophenyl)-2-((4,4,5,5-tetramethyl-1,3,2-dioxa borolan-2-yl)methyl)but-3-en-1-yl)oxy)triethylsilane (8d) Prepared according to the general procedure. The crude mixture was purified by column chromatography (hexane:ethyl acetate $=80: 1$ to $60: 1$ ) to give compound $\mathbf{8 d}$ in $80 \%$ yield $(35 \mathrm{mg})$ as colorless oil. ${ }^{1} \mathrm{H} \mathrm{NMR}\left(600 \mathrm{MHz}, \mathrm{CDCl}_{3}\right) \delta 7.23(\mathrm{~d}, J=8.5 \mathrm{~Hz}, 2 \mathrm{H})$, 7.19 (d, $J=8.4 \mathrm{~Hz}, 2 \mathrm{H}), 5.74$ (ddd, $J=17.1,10.3,8.2 \mathrm{~Hz}, 1 \mathrm{H}), 4.93(\mathrm{~d}, J=10.7 \mathrm{~Hz}, 1 \mathrm{H})$, $4.86(\mathrm{~d}, J=17.3 \mathrm{~Hz}, 1 \mathrm{H}), 4.57(\mathrm{~d}, J=5.1 \mathrm{~Hz}, 1 \mathrm{H}), 2.47-2.51(\mathrm{~m}, 1 \mathrm{H}), 1.21(\mathrm{~s}, 6 \mathrm{H})$, $1.19(\mathrm{~s}, 6 \mathrm{H}), 0.84-0.90(\mathrm{~m}, 10 \mathrm{H}), 0.76(\mathrm{dd}, J=15.3,10.0 \mathrm{~Hz}, 1 \mathrm{H}), 0.33-0.53(\mathrm{~m}, 6 \mathrm{H})$. ${ }^{13} \mathrm{C}$ NMR $\left(151 \mathrm{MHz}, \mathrm{CDCl}_{3}\right) \delta{ }^{13} \mathrm{C}$ NMR $\left(151 \mathrm{MHz}, \mathrm{CDCl}_{3}\right) \delta 143.0,140.1,132.7,128.5$, 128.0, 115.7, 83.3, 78.7, 48.4, 25.3, 25.1, 13.7, 7.2, 5.2. HRMS $\left(\mathrm{ESI}^{+}\right): \mathrm{m} / \mathrm{z}$ for $\mathrm{C}_{23} \mathrm{H}_{38} \mathrm{BO}_{3} \mathrm{NaSiCl}[\mathrm{M}+\mathrm{Na}]^{+}$calcd. 459.2270 , found: 459.2259 . 


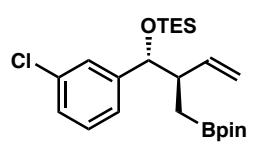

rac- (((1R,2R)-1-(3-chlorophenyl)-2-((4,4,5,5-tetramethyl-1,3,2-dioxa borolan-2-yl)methyl)but-3-en-1-yl)oxy)triethylsilane (8e) Prepared according to the general procedure. The crude mixture was purified by column chromatography (hexane:ethyl acetate $=80: 1$ to $60: 1$ ) to give compound $8 \mathbf{e}$ in $85 \%$ yield $(37 \mathrm{mg})$ as colorless oil. ${ }^{1} \mathrm{H}$ NMR $\left(600 \mathrm{MHz}, \mathrm{CDCl}_{3}\right) \delta 7.22-7.26(\mathrm{~m}, 1 \mathrm{H}), 7.15-$ $7.20(\mathrm{~m}, 2 \mathrm{H}), 7.13(\mathrm{~d}, J=6.8 \mathrm{~Hz}, 1 \mathrm{H}), 5.74(\mathrm{ddd}, J=17.5,10.3,8.2 \mathrm{~Hz}, 1 \mathrm{H}), 4.94(\mathrm{~d}, J=$ $10.1 \mathrm{~Hz}, 1 \mathrm{H}), 4.87$ (d, $J=17.3 \mathrm{~Hz}, 1 \mathrm{H}), 4.57$ (d, $J=5.0 \mathrm{~Hz}, 1 \mathrm{H}), 2.48-2.53(\mathrm{~m}, 1 \mathrm{H})$, $1.21(\mathrm{~s}, 6 \mathrm{H}), 1.20(\mathrm{~s}, 6 \mathrm{H}), 0.90(\mathrm{dd}, J=15.6,4.8 \mathrm{~Hz}, 1 \mathrm{H}), 0.86(\mathrm{t}, J=7.9 \mathrm{~Hz}, 9 \mathrm{H}), 0.77$ $(\mathrm{dd}, J=15.3,10.1 \mathrm{~Hz}, 1 \mathrm{H}), 0.34-0.53(\mathrm{~m}, 6 \mathrm{H}) .{ }^{13} \mathrm{C} \mathrm{NMR}\left(151 \mathrm{MHz}, \mathrm{CDCl}_{3}\right) \delta 146.6$, 140.0, 133.8, 129.1, 127.30, 127.26, 125.4, 115.7, 83.4, 78.7, 48.4, 25.3, 25.1, 13.7, 7.1, 5.2. $\mathrm{HRMS}\left(\mathrm{ESI}^{+}\right): \mathrm{m} / \mathrm{z}$ for $\mathrm{C}_{23} \mathrm{H}_{39} \mathrm{BClO}_{3} \mathrm{Si}[\mathrm{M}+\mathrm{H}]^{+}$calcd. 437.2450, found: 437.2383.

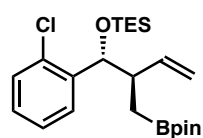

rac-(((1R,2R)-1-(2-chlorophenyl)-2-((4,4,5,5-tetramethyl-1,3,2-dioxabor olan-2-yl)methyl)but-3-en-1-yl)oxy)triethylsilane (8f) Prepared according to the general procedure. The crude mixture was purified by column chromatography (hexane:ethyl acetate $=80: 1$ to $60: 1$ ) to give compound 8 f in $71 \%$ yield $(31 \mathrm{mg})$ as colorless oil. ${ }^{1} \mathrm{H}$ NMR $\left(600 \mathrm{MHz}, \mathrm{CDCl}_{3}\right) \delta 7.46(\mathrm{~d}, J=7.7 \mathrm{~Hz}, 1 \mathrm{H}), 7.25(\mathrm{~d}$, $J=7.4 \mathrm{~Hz}, 1 \mathrm{H}), 7.20(\mathrm{dd}, J=7.4,7.4 \mathrm{~Hz}, 1 \mathrm{H}), 7.12$ (dd, $J=7.4,7.4 \mathrm{~Hz}, 1 \mathrm{H}), 5.82$ (ddd, $J=17.6,10.4,9.4 \mathrm{~Hz}, 1 \mathrm{H}), 5.06(\mathrm{~d}, J=4.3 \mathrm{~Hz}, 1 \mathrm{H}), 4.90(\mathrm{~d}, J=10.3 \mathrm{~Hz}, 1 \mathrm{H}), 4.81(\mathrm{~d}, J$ $=17.3 \mathrm{~Hz}, 1 \mathrm{H}), 2.55-2.60(\mathrm{~m}, 1 \mathrm{H}), 1.21(\mathrm{~s}, 6 \mathrm{H}), 1.19(\mathrm{~s}, 6 \mathrm{H}), 1.03(\mathrm{dd}, J=14.9,10.8$ $\mathrm{Hz}, 1 \mathrm{H}), 0.83-0.89(\mathrm{~m}, 10 \mathrm{H}), 0.40-0.54(\mathrm{~m}, 6 \mathrm{H}) .{ }^{13} \mathrm{C} \mathrm{NMR}\left(151 \mathrm{MHz}, \mathrm{CDCl}_{3}\right) \delta$ 142.2, 139.9, 132.1, 129.6, 129.0, 128.2, 126.5, 115.7, 83.3, 75.1, 46.9, 25.4, 25.1, 14.3, 7.1, 5.1. HRMS $\left(\mathrm{ESI}^{+}\right): \mathrm{m} / \mathrm{z}$ for $\mathrm{C}_{23} \mathrm{H}_{38} \mathrm{BO}_{3} \mathrm{NaSiCl}[\mathrm{M}+\mathrm{Na}]^{+}$calcd. 459.2270, found: 459.2260 .

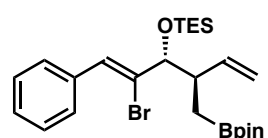

rac-(((3R,4R,Z)-2-bromo-1-phenyl-4-((4,4,5,5-tetramethyl-1,3,2-diox aborolan-2-yl)methyl)hexa-1,5-dien-3-yl)oxy)triethylsilane

Prepared according to the general procedure. The crude mixture was purified by column chromatography (hexane:ethyl acetate $=80: 1$ to $60: 1$ ) to give compound $8 \mathrm{~g}$ in $87 \%$ yield (44 mg) as colorless oil. ${ }^{1} \mathrm{H} \mathrm{NMR}\left(600 \mathrm{MHz}, \mathrm{CDCl}_{3}\right) \delta 7.56$ $(\mathrm{d}, J=7.5 \mathrm{~Hz}, 2 \mathrm{H}), 7.35$ (dd, $J=7.5,7.5 \mathrm{~Hz}, 2 \mathrm{H}), 7.28$ (dd, $J=7.4,7.4 \mathrm{~Hz}, 1 \mathrm{H}), 7.00$ (s, 1H), 5.84 (ddd, $J=17.4,10.3,8.1 \mathrm{~Hz}, 1 \mathrm{H}), 5.04$ (d, $J=17.3 \mathrm{~Hz}, 1 \mathrm{H}), 5.01$ (d, $J=10.3$ $\mathrm{Hz}, 1 \mathrm{H}), 4.25(\mathrm{~d}, J=5.3 \mathrm{~Hz}, 1 \mathrm{H}), 2.78-2.83(\mathrm{~m}, 1 \mathrm{H}), 1.234(\mathrm{~s}, 6 \mathrm{H}), 1.228(\mathrm{~s}, 6 \mathrm{H}), 0.87$ $-0.99(\mathrm{~m}, 11 \mathrm{H}), 0.51-0.68(\mathrm{~m}, 6 \mathrm{H}) .{ }^{13} \mathrm{C} \mathrm{NMR}\left(151 \mathrm{MHz}, \mathrm{CDCl}_{3}\right) \delta 139.4,136.1,129.9$, $129.4,128.43,128.41,128.1,116.0,83.4,82.2$, 44.1, 25.3, 25.2, 14.4, 7.3, 5.3. HRMS $\left(\mathrm{ESI}^{+}\right): \mathrm{m} / \mathrm{z}$ for $\mathrm{C}_{25} \mathrm{H}_{40} \mathrm{BO}_{3} \mathrm{NaSiBr}[\mathrm{M}+\mathrm{Na}]^{+}$calcd. 529.1921, found: 529.1932. 


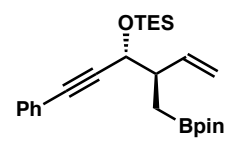

rac-Triethyl(((3R,4R)-1-phenyl-4-((4,4,5,5-tetramethyl-1,3,2-dioxabor olan-2-yl)methyl)hex-5-en-1-yn-3-yl)oxy)silane

(8h) Prepared

according to the general procedure. The crude mixture was purified by column chromatography (hexane:ethyl acetate $=80: 1$ to $60: 1$ ) to give compound $\mathbf{8 h}$ in $82 \%$ yield $(35 \mathrm{mg})$ as colorless oil. ${ }^{1} \mathrm{H}$ NMR (400 $\left.\mathrm{MHz}, \mathrm{CDCl}_{3}\right) \delta 7.36-7.38(\mathrm{~m}, 2 \mathrm{H}), 7.24-$ $7.27(\mathrm{~m}, 3 \mathrm{H}), 5.86$ (ddd, $J=17.8,10.2,9.2 \mathrm{~Hz}, 1 \mathrm{H}), 5.11$ (d, $J=17.2 \mathrm{~Hz}, 1 \mathrm{H}), 5.04$ (d, $J$ $=10.3 \mathrm{~Hz}, 1 \mathrm{H}), 4.51(\mathrm{~d}, J=5.1 \mathrm{~Hz}, 1 \mathrm{H}), 2.57-2.64(\mathrm{~m}, 1 \mathrm{H}), 1.21(\mathrm{~s}, 6 \mathrm{H}), 1.20(\mathrm{~s}, 6 \mathrm{H})$, $0.95-1.05(\mathrm{~m}, 11 \mathrm{H}), 0.55-0.73(\mathrm{~m}, 6 \mathrm{H}) .{ }^{13} \mathrm{C} \mathrm{NMR}\left(101 \mathrm{MHz}, \mathrm{CDCl}_{3}\right) \delta 140.1,131.9$, 128.5, 128.3, 123.6, 116.0, 90.3, 85.4, 83.3, 67.8, 47.1, 25.4, 25.0, 7.2, 5.3. HRMS (ESI ${ }^{+}$): $\mathrm{m} / \mathrm{z}$ for $\mathrm{C}_{25} \mathrm{H}_{39} \mathrm{BO}_{3} \mathrm{NaSi}[\mathrm{M}+\mathrm{Na}]^{+}$calcd. 449.2659, found: 449.2681.

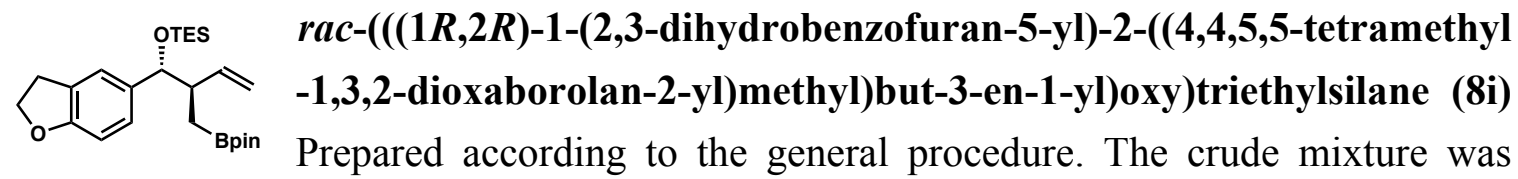
purified by column chromatography (hexane:ethyl acetate $=80: 1$ to $60: 1$ ) to give compound $8 \mathbf{i}$ in $85 \%$ yield (38 mg) as colorless oil. ${ }^{1} \mathrm{H}$ NMR $\left(600 \mathrm{MHz}, \mathrm{CDCl}_{3}\right) \delta 7.10(\mathrm{~s}$, $1 \mathrm{H}), 6.96(\mathrm{~d}, J=8.1 \mathrm{~Hz}, 1 \mathrm{H}), 6.65$ (d, $J=8.1 \mathrm{~Hz}, 1 \mathrm{H}), 5.79$ (ddd, $J=17.8,10.3,8.3 \mathrm{~Hz}$, $1 \mathrm{H}), 4.94(\mathrm{~d}, J=9.9 \mathrm{~Hz}, 1 \mathrm{H}), 4.80-5.11(\mathrm{~m}, 2 \mathrm{H}), 4.54(\mathrm{t}, J=8.7 \mathrm{~Hz}, 2 \mathrm{H}), 4.47(\mathrm{~d}, J=$ $5.8 \mathrm{~Hz}, 1 \mathrm{H}), 3.14-3.19(\mathrm{~m}, 2 \mathrm{H}), 2.48-2.52(\mathrm{~m}, 1 \mathrm{H}), 1.20(\mathrm{~s}, 6 \mathrm{H}), 1.19(\mathrm{~s}, 6 \mathrm{H}), 0.81-$ $0.89(\mathrm{~m}, 10 \mathrm{H}), 0.73(\mathrm{dd}, J=15.3,10.3 \mathrm{~Hz}, 1 \mathrm{H}), 0.35-0.52(\mathrm{~m}, 6 \mathrm{H}) .{ }^{13} \mathrm{C}$ NMR $(101$ $\left.\mathrm{MHz}, \mathrm{CDCl}_{3}\right) \delta 159.3,141.1,136.7,127.0,126.3,123.8,115.1,108.3,83.2,79.3,71.5$, 48.6, 30.1, 25.3, 25.1, 7.2, 5.2. HRMS $\left(\mathrm{ESI}^{+}\right): \mathrm{m} / \mathrm{z}$ for $\mathrm{C}_{25} \mathrm{H}_{41} \mathrm{BO}_{4} \mathrm{NaSi}[\mathrm{M}+\mathrm{Na}]^{+}$calcd. 467.2765, found: 467.2778 .

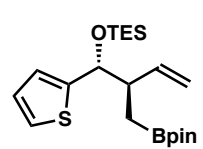

rac-Triethyl(((1R,2R)-2-((4,4,5,5-tetramethyl-1,3,2-dioxaborolan-2-yl)m ethyl)-1-(thiophen-2-yl)but-3-en-1-yl)oxy)silane (8j) Prepared according to the general procedure. The crude mixture was purified by column chromatography (hexane:ethyl acetate $=80: 1$ to $60: 1$ ) to give compound $\mathbf{8 j}$ in $88 \%$ yield $(36 \mathrm{mg})$ as colorless oil. ${ }^{1} \mathrm{H} \mathrm{NMR}\left(600 \mathrm{MHz}, \mathrm{CDCl}_{3}\right) \delta 7.16(\mathrm{~d}, J=4.9 \mathrm{~Hz}, 1 \mathrm{H}), 6.89-$ $6.90(\mathrm{~m}, 1 \mathrm{H}), 6.85(\mathrm{~d}, J=3.3 \mathrm{~Hz}, 1 \mathrm{H}), 5.81-5.87(\mathrm{~m}, 1 \mathrm{H}), 4.98-5.01(\mathrm{~m}, 2 \mathrm{H}), 4.85(\mathrm{~d}$, $J=5.8 \mathrm{~Hz}, 1 \mathrm{H}), 2.57-2.62(\mathrm{~m}, 1 \mathrm{H}), 1.21(\mathrm{~s}, 6 \mathrm{H}), 1.20(\mathrm{~s}, 6 \mathrm{H}), 0.93(\mathrm{dd}, J=15.5,4.3 \mathrm{~Hz}$, $1 \mathrm{H}), 0.88$ (t, $J=7.9 \mathrm{~Hz}, 9 \mathrm{H}), 0.78$ (dd, $J=15.4,10.2 \mathrm{~Hz}, 1 \mathrm{H}), 0.53$ (q, $J=7.7 \mathrm{~Hz}, 6 \mathrm{H})$. ${ }^{13} \mathrm{C}$ NMR $\left(151 \mathrm{MHz}, \mathrm{CDCl}_{3}\right) \delta 148.8,140.5,126.2,124.04,123.99,115.7,83.3,75.6$, 48.9, 25.3, 25.1, 13.5, 7.2, 5.2. HRMS $\left(\mathrm{ESI}^{+}\right): \mathrm{m} / \mathrm{z}$ for $\mathrm{C}_{21} \mathrm{H}_{37} \mathrm{BO}_{3} \mathrm{NaSiS}[\mathrm{M}+\mathrm{Na}]^{+}$calcd. 431.2223, found: 431.2234 . 


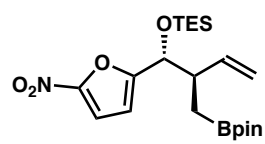

rac-Triethyl(((1R,2R)-1-(5-nitrofuran-2-yl)-2-((4,4,5,5-tetramethyl-1 ,3,2-dioxaborolan-2-yl)methyl)but-3-en-1-yl)oxy)silane (8k) Prepared

according to the general procedure. The crude mixture was purified by column chromatography (hexane:ethyl acetate $=80: 1$ to $60: 1$ ) to give compound $\mathbf{8 k}$ in $98 \%$ yield (43 mg) as white solid. ${ }^{1} \mathrm{H}$ NMR $\left(600 \mathrm{MHz}, \mathrm{CDCl}_{3}\right) \delta 7.24(\mathrm{~d}, J=3.5 \mathrm{~Hz}, 1 \mathrm{H}), 6.42$ $(\mathrm{d}, J=3.7 \mathrm{~Hz}, 1 \mathrm{H}), 5.76(\mathrm{ddd}, J=17.0,10.5,8.4 \mathrm{~Hz}, 1 \mathrm{H}), 4.96-5.00(\mathrm{~m}, 2 \mathrm{H}), 4.75(\mathrm{~d}, J$ $=4.5 \mathrm{~Hz}, 1 \mathrm{H}), 2.71-2.76(\mathrm{~m}, 1 \mathrm{H}), 1.22(\mathrm{~s}, 6 \mathrm{H}), 1.21(\mathrm{~s}, 6 \mathrm{H}), 0.96(\mathrm{dd}, J=15.5,4.4 \mathrm{~Hz}$, $1 \mathrm{H}), 0.87-0.92(\mathrm{~m}, 10 \mathrm{H}), 0.58(\mathrm{q}, J=7.8 \mathrm{~Hz}, 6 \mathrm{H}) .{ }^{13} \mathrm{C} \mathrm{NMR}\left(151 \mathrm{MHz}, \mathrm{CDCl}_{3}\right) \delta$ 161.7, 151.6, 138.6, 116.7, 112.8, 110.2, 83.6, 73.2, 46.3, 25.3, 25.1, 13.6, 7.1, 5.0. HRMS (ESI ${ }^{+}$): m/z for $\mathrm{C}_{21} \mathrm{H}_{36} \mathrm{BNO}_{6} \mathrm{NaSi}[\mathrm{M}+\mathrm{Na}]^{+}$calcd. 460.2303, found: 460.2318.

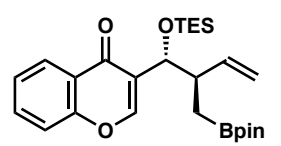

rac-3-((1R,2R)-2-((4,4,5,5-tetramethyl-1,3,2-dioxaborolan-2-yl)meth yl)-1-((triethylsilyl)oxy)but-3-en-1-yl)-4H-chromen-4-one

purified by column chromatography (hexane:ethyl acetate $=80: 1$ to $60: 1$ ) to give compound $3 \mathrm{I}$ in 98\% yield (46 mg) as colorless oil. ${ }^{1} \mathrm{H} \mathrm{NMR}\left(600 \mathrm{MHz}, \mathrm{CDCl}_{3}\right) \delta 8.21$ $(\mathrm{d}, J=7.9 \mathrm{~Hz}, 1 \mathrm{H}), 7.85(\mathrm{~s}, 1 \mathrm{H}), 7.63(\mathrm{dd}, J=8.4,8.4 \mathrm{~Hz}, 1 \mathrm{H}), 7.43(\mathrm{~d}, J=8.4 \mathrm{~Hz}, 1 \mathrm{H})$, 7.38 (dd, $J=7.5,7.5 \mathrm{~Hz}, 1 \mathrm{H}), 5.73$ (ddd, $J=17.5,9.7,9.7 \mathrm{~Hz}, 1 \mathrm{H}), 5.06$ (d, $J=3.1 \mathrm{~Hz}$, 1H), 4.93 (dd, $J=10.3,1.7 \mathrm{~Hz}, 1 \mathrm{H}), 4.81$ (d, $J=17.3 \mathrm{~Hz}, 1 \mathrm{H}), 2.61-2.66$ (m, 1H), 1.20 (s, 6H), 1.18 (s, 6H), 1.09 (dd, $J=15.1,10.8 \mathrm{~Hz}, 1 \mathrm{H}), 1.01$ (dd, $J=15.1,4.2 \mathrm{~Hz}, 1 \mathrm{H})$, $0.91(\mathrm{t}, J=8.0 \mathrm{~Hz}, 9 \mathrm{H}), 0.59$ (q, $J=7.9 \mathrm{~Hz}, 6 \mathrm{H}) .{ }^{13} \mathrm{C} \mathrm{NMR}\left(\mathrm{APT}, 101 \mathrm{MHz}, \mathrm{CDCl}_{3}\right) \delta$ $176.5,156.6,154.9,139.2$, 133.6, 127.0, 126.3, 125.2, 124.2, 118.4, 116.5, 83.3, 71.0, 45.7, 25.3, 25.1, 7.3, 5.2. HRMS $\left(\mathrm{ESI}^{+}\right): \mathrm{m} / \mathrm{z}$ for $\mathrm{C}_{26} \mathrm{H}_{39} \mathrm{BO}_{5} \mathrm{NaSi}[\mathrm{M}+\mathrm{Na}]^{+}$calcd. 493.2558, found: 493.2552 .

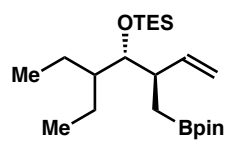

rac-Triethyl(((3R,4S)-5-ethyl-3-((4,4,5,5-tetramethyl-1,3,2-dioxaborol an-2-yl)methyl)hept-1-en-4-yl)oxy)silane (8m) Prepared according to the general procedure. The crude mixture was purified by column chromatography (hexane:ethyl acetate $=80: 1$ to $60: 1$ ) to give compound $\mathbf{8 m}$ in $76 \%$ yield $(30 \mathrm{mg})$ as colorless oil. ${ }^{1} \mathrm{H} \mathrm{NMR}\left(600 \mathrm{MHz}, \mathrm{CDCl}_{3}\right) \delta 5.86(\mathrm{ddd}, J=17.4,10.3,8.7 \mathrm{~Hz}$, $1 \mathrm{H}), 4.98(\mathrm{dd}, J=17.3,1.1 \mathrm{~Hz}, 1 \mathrm{H}), 4.92(\mathrm{dd}, J=10.2,1.5 \mathrm{~Hz} 1 \mathrm{H}), 3.60(\mathrm{dd}, J=4.0,4.0$ $\mathrm{Hz}, 1 \mathrm{H}), 2.46-2.49(\mathrm{~m}, 1 \mathrm{H}), 1.44-1.51(\mathrm{~m}, 1 \mathrm{H}), 1.38-1.44(\mathrm{~m}, 1 \mathrm{H}), 1.16-1.29(\mathrm{~m}$, $15 \mathrm{H}), 0.94-0.97(\mathrm{~m}, 10 \mathrm{H}), 0.82-0.89(\mathrm{~m}, 7 \mathrm{H}), 0.60(\mathrm{q}, J=8.0 \mathrm{~Hz}, 6 \mathrm{H}) .{ }^{13} \mathrm{C} \mathrm{NMR}(151$ $\left.\mathrm{MHz}, \mathrm{CDCl}_{3}\right) \delta 142.0,114.4,83.3,78.4,46.2,43.7,25.3,25.2,22.8,22.2,12.5,12.1,7.5$, 5.9. HRMS (ESI $\left.{ }^{+}\right): \mathrm{m} / \mathrm{z}$ for $\mathrm{C}_{22} \mathrm{H}_{46} \mathrm{BO}_{3} \mathrm{Si}[\mathrm{M}+\mathrm{H}]^{+}$calcd. 397.3309, found: 397.3290 . 


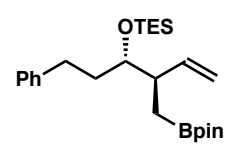

rac-Triethyl(((3S,4R)-1-phenyl-4-((4,4,5,5-tetramethyl-1,3,2-dioxaboro lan-2-yl)methyl)hex-5-en-3-yl)oxy)silane (8n) Prepared according to the general procedure. The crude mixture was purified by column chromatography (hexane:ethyl acetate $=80: 1$ to $60: 1$ ) to give compound $\mathbf{8 n}$ in $88 \%$ yield $(38 \mathrm{mg})$ as colorless oil. ${ }^{1} \mathrm{H} \mathrm{NMR}\left(600 \mathrm{MHz}, \mathrm{CDCl}_{3}\right) \delta 7.27(\mathrm{dd}, J=8.0,7.1 \mathrm{~Hz}, 2 \mathrm{H})$, $7.15-7.18(\mathrm{~m}, 3 \mathrm{H}), 5.77-5.83(\mathrm{~m}, 1 \mathrm{H}), 5.04(\mathrm{~d}, J=17.3 \mathrm{~Hz}, 1 \mathrm{H}), 5.01(\mathrm{~d}, J=10.2 \mathrm{~Hz}$, $1 \mathrm{H}), 3.67-3.69(\mathrm{~m}, 1 \mathrm{H}), 2.64-2.67(\mathrm{~m}, 1 \mathrm{H}), 2.57-2.60(\mathrm{~m}, 1 \mathrm{H}), 2.48-2.51(\mathrm{~m}, 1 \mathrm{H})$, $1.69-1.74(\mathrm{~m}, 2 \mathrm{H}), 1.223(\mathrm{~s}, 6 \mathrm{H}), 1.218(\mathrm{~s}, 6 \mathrm{H}), 0.98-1.02(\mathrm{~m}, 10 \mathrm{H}), 0.91(\mathrm{dd}, J=15.5$, $10.2 \mathrm{~Hz}, 1 \mathrm{H}), 0.61$ (q, $J=7.9 \mathrm{~Hz}, 6 \mathrm{H}) .{ }^{13} \mathrm{C} \mathrm{NMR}\left(101 \mathrm{MHz}, \mathrm{CDCl}_{3}\right) \delta 143.1,140.6$, 128.7, 128.6, 125.9, 115.3, 83.3, 76.3, 45.2, 36.8, 32.6, 25.3, 25.1, 7.4, 5.7. HRMS (ESI ${ }^{+}$): $\mathrm{m} / \mathrm{z}$ for $\mathrm{C}_{25} \mathrm{H}_{44} \mathrm{BO}_{3} \mathrm{Si}[\mathrm{M}+\mathrm{H}]^{+}$calcd. 431.3153, found: 431.3172 .

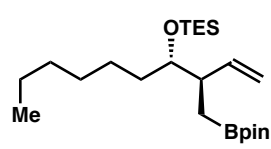

rac-Triethyl(((3R,4S)-3-((4,4,5,5-tetramethyl-1,3,2-dioxaborolan-2-y

l)methyl)dec-1-en-4-yl)oxy)silane (8o) Prepared according to the general procedure. The crude mixture was purified by column chromatography (hexane:ethyl acetate $=80: 1$ to $60: 1$ ) to give compound $\mathbf{8 0}$ in $71 \%$ yield $(29 \mathrm{mg})$ as colorless oil. ${ }^{1} \mathrm{H} \mathrm{NMR}\left(600 \mathrm{MHz}, \mathrm{CDCl}_{3}\right) \delta 5.77$ (ddd, $J=17.1,10.4,8.4 \mathrm{~Hz}$, $1 \mathrm{H}), 4.96-5.00(\mathrm{~m}, 2 \mathrm{H}), 3.56-3.59(\mathrm{~m}, 1 \mathrm{H}), 2.37-2.42(\mathrm{~m}, 1 \mathrm{H}), 1.21-1.41(\mathrm{~m}, 22 \mathrm{H})$, $0.94-1.00(\mathrm{~m}, 10 \mathrm{H}), 0.86-0.91(\mathrm{~m}, 4 \mathrm{H}), 0.59(\mathrm{q}, J=7.9 \mathrm{~Hz}, 6 \mathrm{H}) .{ }^{13} \mathrm{C}$ NMR $(101 \mathrm{MHz}$, $\left.\mathrm{CDCl}_{3}\right) \delta 140.8,115.0,83.2,76.7,45.1,35.0,32.2,29.8,26.1,25.3,25.2,23.0,14.5,7.4$, 5.6. HRMS (ESI $\left.{ }^{+}\right): \mathrm{m} / \mathrm{z}$ for $\mathrm{C}_{23} \mathrm{H}_{48} \mathrm{BO}_{3} \mathrm{Si}[\mathrm{M}+\mathrm{H}]^{+}$calcd. 411.3466 , found: 411.3457.

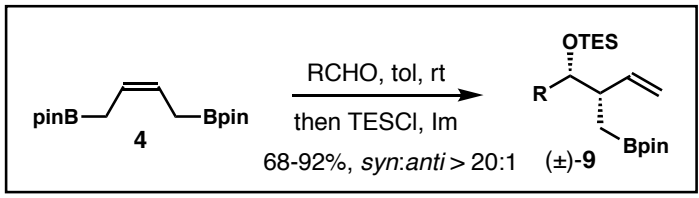

General procedure for synthesis of TES-protected homoallylic alcohols ( \pm )-9: To a reaction vial containing a Teflon-coated magnetic stirring bar was added freshly distilled aldehyde $(0.1 \mathrm{mmol}, 1.0$ equiv). Toluene $(0.3 \mathrm{~mL})$ was added to the vial followed by addition of allylboronate 3 (40 $\mathrm{mg}, 0.13 \mathrm{mmol}, 1.3$ equiv). The reaction mixture was allowed to stir at ambient temperature for $12 \mathrm{~h}$. After complete consumption of the aldehyde, TESCl (23 mg, $0.15 \mathrm{mmol}, 1.5$ equiv), imidazole (14 mg, $0.2 \mathrm{mmol}, 2.0$ equiv) and DMF $(0.2 \mathrm{ml})$ were added to the mixture. The reaction mixture was stirred at ambient temperature for additional $4 \mathrm{~h}$. Then brine $(1 \mathrm{~mL})$ and $\mathrm{Et}_{2} \mathrm{O}(0.5 \mathrm{~mL})$ were added to the vial. The organic layer was separated and the aqueous layer was extracted with $\mathrm{Et}_{2} \mathrm{O}(3 \mathrm{x}$ $1 \mathrm{~mL}$ ). The combined organic extracts were dried over anhydrous magnesium sulfate, 
filtered, and concentrated under reduced pressure. Purification of the crude product was performed by flash chromatography (gradient elution with hexane and ethyl acetate) to provide product $( \pm)-9$.

OTES
an-2-yl)methyl)but-3-en-1-yl)oxy)silane (9a) Prepared according to the
general procedure. The crude mixture was purified by column chromatography (hexane:ethyl acetate $=80: 1$ to $60: 1$ ) to give compound 9a in $80 \%$ yield (32 mg) as colorless oil. ${ }^{1} \mathrm{H}$ NMR (400 MHz, $\left.\mathrm{CDCl}_{3}\right) \delta 7.26-7.28(\mathrm{~m}, 4 \mathrm{H}), 7.18-7.23$ $(\mathrm{m}, 1 \mathrm{H}), 5.69(\mathrm{ddd}, J=17.7,10.9,8.2 \mathrm{~Hz}, 1 \mathrm{H}), 4.89-4.93(\mathrm{~m}, 2 \mathrm{H}), 4.52(\mathrm{~d}, J=6.0 \mathrm{~Hz}$, $1 \mathrm{H}), 2.56-2.64(\mathrm{~m}, 1 \mathrm{H}), 1.23(\mathrm{~s}, 6 \mathrm{H}), 1.21(\mathrm{~s}, 6 \mathrm{H}), 1.07(\mathrm{dd}, J=15.2,4.4 \mathrm{~Hz}, 1 \mathrm{H}), 0.84$ $-0.90(\mathrm{~m}, 10 \mathrm{H}), 0.44-0.55(\mathrm{~m}, 6 \mathrm{H}) .{ }^{13} \mathrm{C} \mathrm{NMR}\left(\mathrm{APT}, 101 \mathrm{MHz}, \mathrm{CDCl}_{3}\right) \delta 144.1,140.9$, 127.8, 127.4, 127.1, 115.1, 83.2, 79.2, 48.3, 25.3, 25.1, 7.2, 5.2. HRMS (ESI $\left.{ }^{+}\right): \mathrm{m} / \mathrm{z}$ for $\mathrm{C}_{23} \mathrm{H}_{40} \mathrm{BO}_{3} \mathrm{Si}[\mathrm{M}+\mathrm{H}]^{+}$calcd. 403.2840, found: 403.2837 .

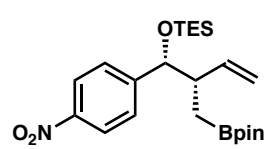

rac-Triethyl(((1R,2S)-1-(4-nitrophenyl)-2-((4,4,5,5-tetramethyl-1,3,2 -dioxaborolan-2-yl)methyl)but-3-en-1-yl)oxy)silane (9b) Prepared according to the general procedure. The crude mixture was purified by column chromatography (hexane:ethyl acetate $=80: 1$ to $60: 1$ ) to give compound $9 \mathrm{~b}$ in $92 \%$ yield (41 mg) as colorless oil. ${ }^{1} \mathrm{H} \mathrm{NMR}\left(600 \mathrm{MHz}, \mathrm{CDCl}_{3}\right) \delta 8.13(\mathrm{~d}, J=8.6 \mathrm{~Hz}, 2 \mathrm{H})$, $7.42(\mathrm{~d}, J=8.5 \mathrm{~Hz}, 2 \mathrm{H}), 5.64(\mathrm{ddd}, J=17.8,10.4,7.9 \mathrm{~Hz}, 1 \mathrm{H}), 4.92(\mathrm{~d}, J=10.3 \mathrm{~Hz}, 1 \mathrm{H})$, $4.87(\mathrm{~d}, J=17.2 \mathrm{~Hz}, 1 \mathrm{H}), 4.63(\mathrm{~d}, J=5.9 \mathrm{~Hz}, 1 \mathrm{H}), 2.55-2.60(\mathrm{~m}, 1 \mathrm{H}), 1.21(\mathrm{~s}, 6 \mathrm{H})$, $1.20(\mathrm{~s}, 6 \mathrm{H}), 0.96(\mathrm{dd}, J=15.3,5.1 \mathrm{~Hz}, 1 \mathrm{H}), 0.83-0.87(\mathrm{~m}, 10 \mathrm{H}), 0.44-0.55(\mathrm{~m}, J=$ $7.5 \mathrm{~Hz}, 6 \mathrm{H}) .{ }^{13} \mathrm{C}$ NMR $\left(101 \mathrm{MHz}, \mathrm{CDCl}_{3}\right) \delta 151.7,147.4,139.7,128.1,123.2,116.2$,

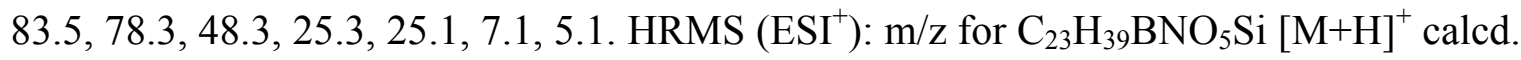
448.2691, found: 448.2694.

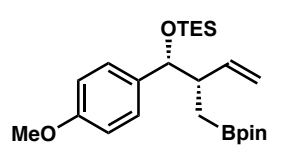

rac-Triethyl(((1R,2S)-1-(4-methoxyphenyl)-2-((4,4,5,5-tetramethyl1,3,2-dioxaborolan-2-yl)methyl)but-3-en-1-yl)oxy)silane

Prepared according to the general procedure. The crude mixture was purified by column chromatography (hexane:ethyl acetate $=80: 1$ to $60: 1$ ) to give compound $9 \mathrm{c}$ in $86 \%$ yield $\left(37 \mathrm{mg}\right.$ ) as colorless oil. ${ }^{1} \mathrm{H} \mathrm{NMR}\left(400 \mathrm{MHz}, \mathrm{CDCl}_{3}\right) \delta 7.16$ $(\mathrm{d}, J=8.4 \mathrm{~Hz}, 2 \mathrm{H}), 6.79$ (d, $J=8.4 \mathrm{~Hz}, 2 \mathrm{H}), 5.66$ (ddd, $J=17.7,10.4,8.4 \mathrm{~Hz}, 1 \mathrm{H}), 4.86$ $-4.90(\mathrm{~m}, 2 \mathrm{H}), 4.45(\mathrm{~d}, J=6.1 \mathrm{~Hz}, 1 \mathrm{H}), 3.78(\mathrm{~s}, 3 \mathrm{H}), 2.52-2.59(\mathrm{~m}, 1 \mathrm{H}), 1.21(\mathrm{~s}, 6 \mathrm{H})$, $1.20(\mathrm{~s}, 6 \mathrm{H}), 1.06(\mathrm{dd}, J=15.3,4.7 \mathrm{~Hz}, 1 \mathrm{H}), 0.79-0.87(\mathrm{~m}, 10 \mathrm{H}), 0.42-0.52(\mathrm{~m}, 6 \mathrm{H})$.

${ }^{13} \mathrm{C} \mathrm{NMR}\left(101 \mathrm{MHz}, \mathrm{CDCl}_{3}\right) \delta 158.8,141.0,136.3,128.5,115.0,113.2,83.2,78.8,55.5$, 
48.4, 25.3, 25.1, 7.2, 5.2. HRMS $\left(\mathrm{ESI}^{+}\right): \mathrm{m} / \mathrm{z}$ for $\mathrm{C}_{24} \mathrm{H}_{41} \mathrm{BO}_{4} \mathrm{NaSi}[\mathrm{M}+\mathrm{Na}]^{+}$calcd. 455.2765, found: 455.2766 .
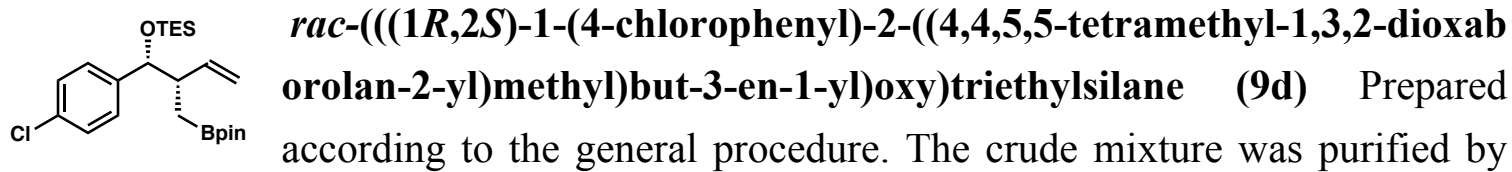

column chromatography (hexane:ethyl acetate $=80: 1$ to $60: 1$ ) to give compound $9 \mathbf{d}$ in $85 \%$ yield $(37 \mathrm{mg})$ as colorless oil. ${ }^{1} \mathrm{H}$ NMR $\left(600 \mathrm{MHz}, \mathrm{CDCl}_{3}\right) \delta 7.23(\mathrm{~d}, J=8.4 \mathrm{~Hz}, 2 \mathrm{H})$, $7.18(\mathrm{~d}, J=8.3 \mathrm{~Hz}, 2 \mathrm{H}), 5.64(\mathrm{ddd}, J=18.2,10.4,7.9 \mathrm{~Hz}, 1 \mathrm{H}), 4.87-4.90$ (m, 2H), 4.49 $(\mathrm{d}, J=5.9 \mathrm{~Hz}, 1 \mathrm{H}), 2.51-2.56(\mathrm{~m}, 1 \mathrm{H}), 1.21(\mathrm{~s}, 6 \mathrm{H}), 1.19(\mathrm{~s}, 6 \mathrm{H}), 1.00(\mathrm{dd}, J=15.4,4.8$ $\mathrm{Hz}, 1 \mathrm{H}), 0.80-0.84(\mathrm{~m}, 10 \mathrm{H}), 0.43-0.53(\mathrm{~m}, 6 \mathrm{H}) .{ }^{13} \mathrm{C}$ NMR (APT, $\left.101 \mathrm{MHz}, \mathrm{CDCl}_{3}\right) \delta$ $142.6,140.5,132.7,128.7,128.0,115.5,83.3,78.5,48.3,25.3,25.1,7.2$, 5.2. HRMS $\left(\mathrm{ESI}^{+}\right): \mathrm{m} / \mathrm{z}$ for $\mathrm{C}_{23} \mathrm{H}_{38} \mathrm{BO}_{3} \mathrm{NaSiCl}[\mathrm{M}+\mathrm{Na}]^{+}$calcd. 459.2270, found: 459.2292.

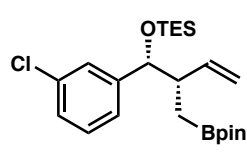

rac-(((1R,2S)-1-(3-chlorophenyl)-2-((4,4,5,5-tetramethyl-1,3,2-dioxab orolan-2-yl)methyl)but-3-en-1-yl)oxy)triethylsilane (9e) Prepared according to the general procedure. The crude mixture was purified by column chromatography (hexane:ethyl acetate $=80: 1$ to $60: 1$ ) to give compound $9 \mathrm{e}$ in $76 \%$ yield $(33 \mathrm{mg})$ as colorless oil. ${ }^{1} \mathrm{H}$ NMR $\left(600 \mathrm{MHz}, \mathrm{CDCl}_{3}\right) \delta 7.25(\mathrm{~d}, J=1.8 \mathrm{~Hz}, 1 \mathrm{H})$, $7.14-7.17$ (m, 2H), $7.10-7.13(\mathrm{~m}, 1 \mathrm{H}), 5.65$ (ddd, $J=16.8,10.6,7.8 \mathrm{~Hz}, 1 \mathrm{H}), 4.88-$ $4.91(\mathrm{~m}, 2 \mathrm{H}), 4.48(\mathrm{~d}, J=5.9 \mathrm{~Hz}, 1 \mathrm{H}), 2.51-2.56(\mathrm{~m}, 1 \mathrm{H}), 1.20(\mathrm{~s}, 6 \mathrm{H}), 1.18(\mathrm{~s}, 6 \mathrm{H})$, $1.00(\mathrm{dd}, J=15.4,4.7 \mathrm{~Hz}, 1 \mathrm{H}), 0.81-0.86(\mathrm{~m}, 10 \mathrm{H}), 0.42-0.52(\mathrm{~m}, 6 \mathrm{H}) .{ }^{13} \mathrm{C} \mathrm{NMR}$ $\left(151 \mathrm{MHz}, \mathrm{CDCl}_{3}\right) \delta 146.3,140.4,133.8,129.1,127.5,127.3,125.6,115.6,83.3,78.5$, 48.3, 25.3, 25.0, 11.8, 7.1, 5.2. $\mathrm{HRMS}\left(\mathrm{ESI}^{+}\right): \mathrm{m} / \mathrm{z}$ for $\mathrm{C}_{23} \mathrm{H}_{38} \mathrm{BO}_{3} \mathrm{NaSiCl}[\mathrm{M}+\mathrm{Na}]^{+}$calcd. 459.2270, found: 459.2291 .

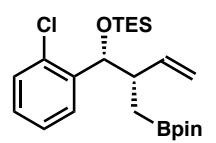

rac-(((1R,2S)-1-(2-chlorophenyl)-2-((4,4,5,5-tetramethyl-1,3,2-dioxabor olan-2-yl)methyl)but-3-en-1-yl)oxy)triethylsilane (9f) Prepared according to the general procedure. The crude mixture was purified by column chromatography (hexane:ethyl acetate $=80: 1$ to $60: 1$ ) to give compound 9 f in $69 \%$ yield $(30 \mathrm{mg})$ as colorless oil. ${ }^{1} \mathrm{H}$ NMR $\left(600 \mathrm{MHz}, \mathrm{CDCl}_{3}\right) \delta 7.51(\mathrm{~d}, J=7.4 \mathrm{~Hz}, 1 \mathrm{H}), 7.25$ (d, $J=7.9 \mathrm{~Hz}, 1 \mathrm{H}), 7.21(\mathrm{dd}, J=7.5,7.5 \mathrm{~Hz}, 1 \mathrm{H}), 7.11-7.20(\mathrm{~m}, 1 \mathrm{H}), 5.75-5.80(\mathrm{~m}, 1 \mathrm{H})$, $5.05(\mathrm{~d}, J=5.3 \mathrm{~Hz}, 1 \mathrm{H}), 4.94(\mathrm{~d}, J=17.2 \mathrm{~Hz}, 1 \mathrm{H}), 4.91$ (d, $J=10.3 \mathrm{~Hz}, 1 \mathrm{H}), 2.58-2.63$ $(\mathrm{m}, 1 \mathrm{H}), 1.19$ (s, 6H), $1.17(\mathrm{~s}, 6 \mathrm{H}), 1.02(\mathrm{dd}, J=15.2,4.1 \mathrm{~Hz}, 1 \mathrm{H}), 0.94(\mathrm{dd}, J=15.2$, $11.0 \mathrm{~Hz}, 1 \mathrm{H}), 0.84(\mathrm{t}, J=8.0 \mathrm{~Hz}, 9 \mathrm{H}), 0.43-0.53(\mathrm{~m}, 6 \mathrm{H}) .{ }^{13} \mathrm{C} \mathrm{NMR}\left(101 \mathrm{MHz}, \mathrm{CDCl}_{3}\right)$ $\delta 142.1,140.8,132.3,129.5,129.1,128.2,126.6,115.4,83.2,74.5,47.4,25.4,25.0$, 7.1, 
5.1. HRMS $\left(\mathrm{ESI}^{+}\right): \mathrm{m} / \mathrm{z}$ for $\mathrm{C}_{23} \mathrm{H}_{38} \mathrm{BO}_{3} \mathrm{NaSiCl}[\mathrm{M}+\mathrm{Na}]^{+}$calcd. 459.2270, found: 459.2292 .

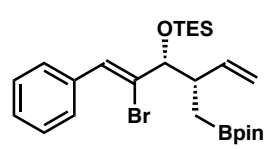

rac-(((3R,4S,Z)-2-bromo-1-phenyl-4-((4,4,5,5-tetramethyl-1,3,2-diox aborolan-2-yl)methyl)hexa-1,5-dien-3-yl)oxy)triethylsilane

Prepared according to the general procedure. The crude mixture was purified by column chromatography (hexane:ethyl acetate $=80: 1$ to $60: 1$ ) to give compound $9 \mathrm{~g}$ in $89 \%$ yield $(45 \mathrm{mg})$ as colorless oil. ${ }^{1} \mathrm{H} \mathrm{NMR}\left(600 \mathrm{MHz}, \mathrm{CDCl}_{3}\right) \delta 7.59$ (d, $J=7.6 \mathrm{~Hz}, 2 \mathrm{H}), 7.35$ (dd, $J=7.6,7.6 \mathrm{~Hz}, 2 \mathrm{H}), 7.29$ (dd, $J=7.4,7.4 \mathrm{~Hz}, 1 \mathrm{H}), 6.98$ (s, 1H), 5.75 (ddd, $J=18.2,10.2,8.4 \mathrm{~Hz}, 1 \mathrm{H}), 5.12$ (d, $J=17.1 \mathrm{~Hz}, 1 \mathrm{H}), 4.99-5.00$ (m, $1 \mathrm{H}), 4.15(\mathrm{~d}, J=6.1 \mathrm{~Hz}, 1 \mathrm{H}), 2.79-2.84(\mathrm{~m}, 1 \mathrm{H}), 1.22(\mathrm{~s}, 6 \mathrm{H}), 1.21(\mathrm{~s}, 6 \mathrm{H}), 1.18(\mathrm{dd}, J$ $=15.4,3.8 \mathrm{~Hz}, 1 \mathrm{H}), 0.97(\mathrm{t}, J=8.0 \mathrm{~Hz}, 9 \mathrm{H}), 0.91(\mathrm{dd}, J=15.4,10.9 \mathrm{~Hz}, 1 \mathrm{H}), 0.64(\mathrm{q}, J$ $=7.7 \mathrm{~Hz}, 6 \mathrm{H}) .{ }^{13} \mathrm{C} \mathrm{NMR}\left(101 \mathrm{MHz}, \mathrm{CDCl}_{3}\right) \delta 140.5,135.9,130.0,129.4,128.5,128.4$, 128.1, 115.7, 83.3, 82.3, 44.6, 25.3, 25.1, 7.3, 5.2. HRMS (ESI $\left.{ }^{+}\right): \mathrm{m} / \mathrm{z}, \mathrm{C}_{25} \mathrm{H}_{40} \mathrm{BO}_{3} \mathrm{NaSiBr}$ $[\mathrm{M}+\mathrm{Na}]^{+}$calcd. 529.1921, found: 529.1947.

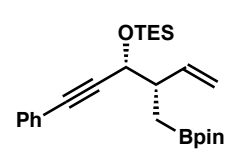

rac-Triethyl(((3R,4S)-1-phenyl-4-((4,4,5,5-tetramethyl-1,3,2-dioxabor olan-2-yl)methyl)hex-5-en-1-yn-3-yl)oxy)silane

(9h) Prepared

according to the general procedure. The crude mixture was purified (hexane:ethyl acetate $=80: 1$ to $60: 1)$ to give compound $9 \mathbf{h}$ in $77 \%$ yield $(33 \mathrm{mg}$ ) as colorless oil. ${ }^{1} \mathrm{H}$ NMR (500 MHz, $\left.\mathrm{CDCl}_{3}\right) \delta 7.38-7.40(\mathrm{~m}, 2 \mathrm{H}), 7.27-7.29(\mathrm{~m}, 3 \mathrm{H})$, 5.97 (ddd, $J=17.9,10.0,8.3 \mathrm{~Hz}, 1 \mathrm{H}), 5.13$ (d, $J=17.2 \mathrm{~Hz}, 1 \mathrm{H}), 5.08$ (d, $J=10.4 \mathrm{~Hz}$, $1 \mathrm{H}), 4.54(\mathrm{~d}, J=5.5 \mathrm{~Hz}, 1 \mathrm{H}), 2.60-2.66(\mathrm{~m}, 1 \mathrm{H}), 1.24(\mathrm{~s}, 6 \mathrm{H}), 1.23(\mathrm{~s}, 6 \mathrm{H}), 1.15$ (dd, $J$ $=15.6,5.1 \mathrm{~Hz}, 1 \mathrm{H}), 0.95-1.05(\mathrm{~m}, 10 \mathrm{H}), 0.60-0.75(\mathrm{~m}, 6 \mathrm{H}) .{ }^{13} \mathrm{C}$ NMR $(126 \mathrm{MHz}$, $\left.\mathrm{CDCl}_{3}\right) \delta 139.9,131.9,128.5,128.3,123.7,116.0,90.1,85.4,83.4,67.8,46.9,25.3,25.1$, 7.2, 5.2. HRMS (ESI $\left.{ }^{+}\right): \mathrm{m} / \mathrm{z}$ for $\mathrm{C}_{25} \mathrm{H}_{40} \mathrm{BO}_{3} \mathrm{Si}[\mathrm{M}+\mathrm{H}]^{+}$calcd. 427.2840, found: 427.2848.

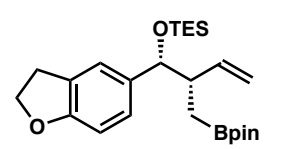

rac-(((1R,2S)-1-(2,3-dihydrobenzofuran-5-yl)-2-((4,4,5,5-tetramethyl -1,3,2-dioxaborolan-2-yl)methyl)but-3-en-1-yl)oxy)triethylsilane (9i)

Prepared according to the general procedure. The crude mixture was purified by column chromatography (hexane:ethyl acetate $=80: 1$ to $60: 1$ ) to give compound $9 \mathbf{i}$ in $81 \%$ yield (36 mg) as colorless oil. ${ }^{1} \mathrm{H}$ NMR (400 MHz, $\left.\mathrm{CDCl}_{3}\right) \delta 7.08(\mathrm{~s}$, $1 \mathrm{H}), 6.95(\mathrm{~d}, J=8.0 \mathrm{~Hz}, 1 \mathrm{H}), 6.65(\mathrm{~d}, J=8.1 \mathrm{~Hz}, 1 \mathrm{H}), 5.62-5.70(\mathrm{~m}, 1 \mathrm{H}), 4.87-4.92$ $(\mathrm{m}, 2 \mathrm{H}), 4.54(\mathrm{t}, J=8.6 \mathrm{~Hz}, 2 \mathrm{H}), 4.43(\mathrm{~d}, J=6.0 \mathrm{~Hz}, 1 \mathrm{H}), 3.17(\mathrm{t}, J=8.6 \mathrm{~Hz}, 2 \mathrm{H}), 2.50-$ $2.57(\mathrm{~m}, 1 \mathrm{H}), 1.21(\mathrm{~s}, 6 \mathrm{H}), 1.20(\mathrm{~s}, 6 \mathrm{H}), 1.07(\mathrm{dd}, J=15.4,4.3 \mathrm{~Hz}, 1 \mathrm{H}), 0.79-0.87$ (m, $10 \mathrm{H}), 0.44-0.52(\mathrm{~m}, 6 \mathrm{H}) .{ }^{13} \mathrm{C} \mathrm{NMR}\left(101 \mathrm{MHz}, \mathrm{CDCl}_{3}\right) \delta 159.3,141.1,136.4,127.2$, 
126.3, 123.9, 114.9, 108.3, 83.2, 79.0, 71.5, 48.4, 30.1, 25.3, 25.1, 7.2, 5.2. HRMS (ESI $\left.{ }^{+}\right)$: $\mathrm{m} / \mathrm{z}$ for $\mathrm{C}_{25} \mathrm{H}_{41} \mathrm{BO}_{4} \mathrm{NaSi}[\mathrm{M}+\mathrm{Na}]^{+}$calcd. 467.2765, found: 467.2770.

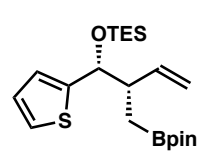

rac-Triethyl(((1R,2S)-2-((4,4,5,5-tetramethyl-1,3,2-dioxaborolan-2-yl)m ethyl)-1-(thiophen-2-yl)but-3-en-1-yl)oxy)silane (9j) Prepared according to the general procedure. The crude mixture was purified by column chromatography (hexane:ethyl acetate $=80: 1$ to $60: 1$ ) to give compound $9 \mathbf{j}$ in $86 \%$ yield (35 mg) as colorless oil. ${ }^{1} \mathrm{H}$ NMR $\left(500 \mathrm{MHz} \mathrm{CDCl}_{3}\right) \delta 7.16(\mathrm{~d}, J=4.9 \mathrm{~Hz}, 1 \mathrm{H}), 6.88$ (dd, $J=3.8,4.5 \mathrm{~Hz} 1 \mathrm{H}), 6.83(\mathrm{~d}, J=2.9 \mathrm{~Hz}, 1 \mathrm{H}), 5.72(\mathrm{ddd}, J=17.6,10.2,7.9 \mathrm{~Hz}, 1 \mathrm{H}), 4.93$ $-4.97(\mathrm{~m}, 2 \mathrm{H}), 4.81(\mathrm{~d}, J=6.3 \mathrm{~Hz}, 1 \mathrm{H}), 2.61-2.67(\mathrm{~m}, 1 \mathrm{H}), 1.23(\mathrm{~s}, 6 \mathrm{H}), 1.21(\mathrm{~s}, 6 \mathrm{H})$, $1.11(\mathrm{dd}, J=15.5,5.0 \mathrm{~Hz}, 1 \mathrm{H}), 0.86-0.91(\mathrm{~m}, 10 \mathrm{H}), 0.47-0.58(\mathrm{~m}, 6 \mathrm{H}) .{ }^{13} \mathrm{C} \mathrm{NMR}$ $\left(126 \mathrm{MHz}, \mathrm{CDCl}_{3}\right) \delta 148.3,140.2,126.1,124.19,124.15,115.6,83.3,75.3,48.6,25.3$, 25.1, 12.5, 7.2, 5.2. HRMS $\left(\mathrm{ESI}^{+}\right): \mathrm{m} / \mathrm{z}, \mathrm{C}_{21} \mathrm{H}_{37} \mathrm{BO}_{3} \mathrm{NaSiS}[\mathrm{M}+\mathrm{Na}]^{+}$calcd. 431.2223, found: 431.2208 .

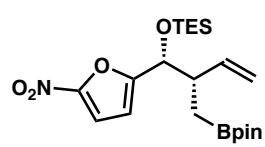

rac-Triethyl(((1R,2S)-2-((4,4,5,5-tetramethyl-1,3,2-dioxaborolan-2-y l)methyl)-1-(thiophen-2-yl)but-3-en-1-yl)oxy)silane (9k) Prepared according to the general procedure. The crude mixture was purified by column chromatography (hexane:ethyl acetate $=80: 1$ to $60: 1$ ) to give compound $9 \mathbf{k}$ in $82 \%$ yield (36 mg) as white solid. ${ }^{1} \mathrm{H}$ NMR $\left(500 \mathrm{MHz}, \mathrm{CDCl}_{3}\right) \delta 7.24(\mathrm{~d}, J=3.6 \mathrm{~Hz}, 1 \mathrm{H}), 6.42$ $(\mathrm{d}, J=3.6 \mathrm{~Hz}, 1 \mathrm{H}), 5.73$ (ddd, $J=17.8,10.3,8.0 \mathrm{~Hz}, 1 \mathrm{H}), 4.99-5.03(\mathrm{~m}, 2 \mathrm{H}), 4.70(\mathrm{~d}, J$ $=6.1 \mathrm{~Hz}, 1 \mathrm{H}), 2.72-2.78(\mathrm{~m}, 1 \mathrm{H}), 1.22(\mathrm{~s}, 6 \mathrm{H}), 1.21(\mathrm{~s}, 6 \mathrm{H}), 1.01(\mathrm{dd}, J=15.6,5.1 \mathrm{~Hz}$, $1 \mathrm{H}), 0.88-0.94(\mathrm{~m}, 10 \mathrm{H}), 0.50-0.61(\mathrm{~m}, 6 \mathrm{H}) .{ }^{13} \mathrm{C}$ NMR $\left(126 \mathrm{MHz}, \mathrm{CDCl}_{3}\right) \delta 161.4$, $151.6,139.0,116.7,112.8,110.4,83.5,72.6,46.2,25.3,25.0,11.9,7.0$, 5.0. HRMS $\left(\mathrm{ESI}^{+}\right): \mathrm{m} / \mathrm{z}$ for $\mathrm{C}_{21} \mathrm{H}_{36} \mathrm{BNO}_{6} \mathrm{NaSi}[\mathrm{M}+\mathrm{Na}]^{+}$calcd. 460.2303 , found: 460.2348 .

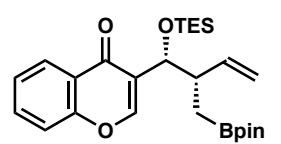

rac-3-((1R,2S)-2-((4,4,5,5-tetramethyl-1,3,2-dioxaborolan-2-yl)meth yl)-1-((triethylsilyl)oxy)but-3-en-1-yl)-4H-chromen-4-one

Prepared according to the general procedure. The crude mixture was purified by column chromatography (hexane:ethyl acetate $=80: 1$ to $60: 1$ ) to give compound 91 in $91 \%$ yield (43 mg) as colorless oil. ${ }^{1} \mathrm{H}$ NMR (400 $\left.\mathrm{MHz}, \mathrm{CDCl}_{3}\right) \delta 8.21$ $(\mathrm{d}, J=8.1 \mathrm{~Hz}, 1 \mathrm{H}), 7.91$ (s, 1H), $7.64(\mathrm{dd}, J=7.8,7.8 \mathrm{~Hz}, 1 \mathrm{H}), 7.44(\mathrm{~d}, J=8.4 \mathrm{~Hz}, 1 \mathrm{H})$, $7.38(\mathrm{dd}, J=7.5,7.5 \mathrm{~Hz}, 1 \mathrm{H}), 5.84-5.93(\mathrm{~m}, 1 \mathrm{H}), 5.05-5.11(\mathrm{~m}, 2 \mathrm{H}), 5.00(\mathrm{~d}, J=10.4$ $\mathrm{Hz}, 1 \mathrm{H}), 2.65-2.71(\mathrm{~m}, 1 \mathrm{H}), 1.15(\mathrm{~s}, 12 \mathrm{H}), 0.89-0.99(\mathrm{~m}, 10 \mathrm{H}), 0.83$ (dd, $J=15.4$, $10.4 \mathrm{~Hz}, 1 \mathrm{H}), 0.57$ (q, $J=7.8 \mathrm{~Hz}, 6 \mathrm{H}) .{ }^{13} \mathrm{C} \mathrm{NMR}\left(\mathrm{APT}, 101 \mathrm{MHz}, \mathrm{CDCl}_{3}\right) \delta 176.6,156.6$, 
154.7, 141.0, 133.6, 126.8, 126.4, 125.2, 124.3, 118.4, 115.6, 83.2, 70.1, 45.8, 25.3, 24.9, 7.2, 5.1. HRMS (ESI ${ }^{+}$): $\mathrm{m} / \mathrm{z}$ for $\mathrm{C}_{26} \mathrm{H}_{40} \mathrm{BO}_{5} \mathrm{Si}[\mathrm{M}+\mathrm{H}]^{+}$calcd. 471.2738, found: 471.2727.

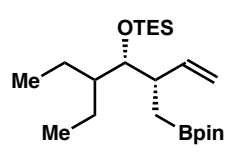

rac-Triethyl(((3S,4S)-5-ethyl-3-((4,4,5,5-tetramethyl-1,3,2-dioxaborola n-2-yl)methyl)hept-1-en-4-yl)oxy)silane (9m) Prepared according to the general procedure. The crude mixture was purified by column chromatography (hexane:ethyl acetate $=80: 1$ to $60: 1$ ) to give compound $9 \mathrm{~m}$ in $71 \%$ yield $(28 \mathrm{mg})$ as colorless oil. ${ }^{1} \mathrm{H}$ NMR $\left(600 \mathrm{MHz}, \mathrm{CDCl}_{3}\right) \delta 5.70(\mathrm{ddd}, J=17.4,10.2,8.7 \mathrm{~Hz}$, $1 \mathrm{H}), 5.00(\mathrm{dd}, J=17.2,0.9 \mathrm{~Hz}, 1 \mathrm{H}), 4.92(\mathrm{dd}, J=10.3,1.6 \mathrm{~Hz}, 1 \mathrm{H}), 3.53(\mathrm{dd}, J=6.8$, $2.7 \mathrm{~Hz}, 1 \mathrm{H}), 2.45-2.50(\mathrm{~m}, 1 \mathrm{H}), 1.47-1.54(\mathrm{~m}, 1 \mathrm{H}), 1.30-1.35(\mathrm{~m}, 2 \mathrm{H}), 1.21-1.26$ $(\mathrm{m}, 13 \mathrm{H}), 1.07-1.16(\mathrm{~m}, 2 \mathrm{H}), 0.96(\mathrm{t}, J=8.0 \mathrm{~Hz}, 9 \mathrm{H}), 0.82-0.88(\mathrm{~m}, 6 \mathrm{H}), 0.75(\mathrm{dd}, J=$ 15.2, $10.7 \mathrm{~Hz}, 1 \mathrm{H}), 0.56-0.65(\mathrm{~m}, 6 \mathrm{H}) .{ }^{13} \mathrm{C} \mathrm{NMR}\left(151 \mathrm{MHz}, \mathrm{CDCl}_{3}\right) \delta 142.4,114.5$, 83.2, 78.2, 45.7, 44.6, 25.3, 25.2, 23.7, 22.1, 12.8, 12.6, 7.5, 5.9. HRMS (ESI $\left.{ }^{+}\right): \mathrm{m} / \mathrm{z}$ for $\mathrm{C}_{22} \mathrm{H}_{46} \mathrm{BO}_{3} \mathrm{Si}[\mathrm{M}+\mathrm{H}]^{+}$calcd. 397.3309, found: 397.3307 .

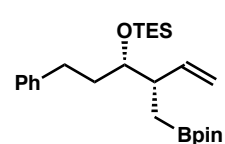

rac-Triethyl(((3S,4S)-1-phenyl-4-((4,4,5,5-tetramethyl-1,3,2-dioxaboro lan-2-yl)methyl)hex-5-en-3-yl)oxy)silane (9n) Prepared according to the general procedure. The crude mixture was purified by column chromatography (hexane: ethyl acetate $=80: 1$ to $60: 1$ ) to give compound $9 \mathrm{n}$ in $86 \%$ yield (37 mg) as colorless oil. ${ }^{1} \mathrm{H}$ NMR $\left(600 \mathrm{MHz} \mathrm{CDCl}_{3}\right) \delta 7.21-7.41$ (dd, $J=7.5,7.5 \mathrm{~Hz}$, 2H), $7.16-7.17(\mathrm{~m}, 3 \mathrm{H}), 5.84$ (ddd, $J=17.8,10.3,7.8 \mathrm{~Hz}, 1 \mathrm{H}), 5.06$ (d, $J=17.3 \mathrm{~Hz}$, $1 \mathrm{H}), 5.01(\mathrm{~d}, J=10.3 \mathrm{~Hz}, 1 \mathrm{H}), 3.65-3.68(\mathrm{~m}, 1 \mathrm{H}), 2.73-2.78(\mathrm{~m}, 1 \mathrm{H}), 2.50-2.59$ (m, 2H), $1.65-1.77(\mathrm{~m}, 2 \mathrm{H}), 1.23(\mathrm{~s}, 6 \mathrm{H}), 1.22$ (s, 6H), $0.95-1.01(\mathrm{~m}, 10 \mathrm{H}), 0.83$ (dd, $J=$ $15.3,9.5 \mathrm{~Hz}, 1 \mathrm{H}), 0.65$ (q, $J=7.9 \mathrm{~Hz}, 6 \mathrm{H}) .{ }^{13} \mathrm{C} \mathrm{NMR}\left(151 \mathrm{MHz}, \mathrm{CDCl}_{3}\right) \delta 143.3,141.1$, 128.7, 128.6, 125.9, 114.9, 83.3, 76.1, 45.3, 36.0, 32.1, 25.3, 25.1, 7.4, 5.7. HRMS (ESI ${ }^{+}$): $\mathrm{m} / \mathrm{z}$ for $\mathrm{C}_{25} \mathrm{H}_{44} \mathrm{BO}_{3} \mathrm{Si}[\mathrm{M}+\mathrm{Na}]^{+}$calcd. 431.3153, found: 431.3165 .

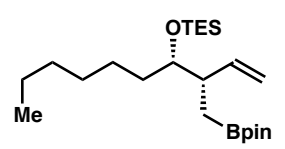

rac-Triethyl(((3S,4S)-3-((4,4,5,5-tetramethyl-1,3,2-dioxaborolan-2-y l)methyl)dec-1-en-4-yl)oxy)silane (9o) Prepared according to the general procedure. The crude mixture was purified (hexane:ethyl acetate $=80: 1$ to $60: 1)$ to give compound 90 in $73 \%$ yield $(30 \mathrm{mg})$ as colorless oil. ${ }^{1} \mathrm{H}$ NMR $\left(500 \mathrm{MHz}, \mathrm{CDCl}_{3}\right) \delta 5.83(\mathrm{ddd}, J=17.7,10.2,7.8 \mathrm{~Hz}, 1 \mathrm{H}), 4.97-5.03(\mathrm{~m}, 2 \mathrm{H})$, $3.56-3.59(\mathrm{~m}, 1 \mathrm{H}), 2.41-2.46(\mathrm{~m}, 1 \mathrm{H}), 1.22-1.40(\mathrm{~m}, 22 \mathrm{H}), 0.86-0.97(\mathrm{~m}, 13 \mathrm{H})$, $0.81(\mathrm{dd}, J=15.4,9.5 \mathrm{~Hz}, 1 \mathrm{H}), 0.60(\mathrm{q}, J=7.9 \mathrm{~Hz}, 6 \mathrm{H}) .{ }^{13} \mathrm{C} \mathrm{NMR}\left(126 \mathrm{MHz}, \mathrm{CDCl}_{3}\right) \delta$ 141.4, 114.6, 83.3, 76.4, 45.3, 33.8, 32.2, 29.9, 25.7, 25.3, 25.1, 23.0, 14.4, 12.4, 7.4, 5.6. HRMS (ESI ${ }^{+}$): $\mathrm{m} / \mathrm{z}$ for $\mathrm{C}_{23} \mathrm{H}_{48} \mathrm{BO}_{3} \mathrm{Si}[\mathrm{M}+\mathrm{H}]^{+}$calcd. 411.3466 , found: 411.3482 . 


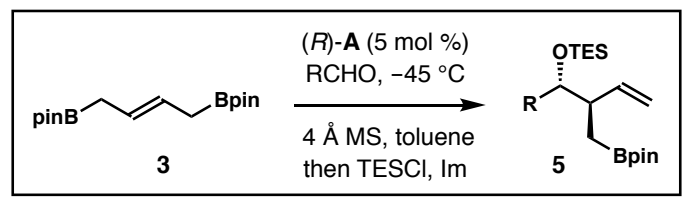

General procedure for synthesis of chiral TES-protected homoallylic alcohols 5: To a reaction flask containing a stir bar, freshly activated $4 \AA$ MS (25 mg) and phosphoric acid $(R)$-A $(3.8 \mathrm{mg}, 0.005 \mathrm{mmol})$, allylboronate $3(0.1 \mathrm{mmol})$ and toluene $(0.3 \mathrm{~mL})$ was added to the flask. The mixture was placed in a $-45^{\circ} \mathrm{C}$ cold bath and stirred for $5 \mathrm{~min}$. Then freshly distilled aldehyde $(0.2 \mathrm{mmol}$, if it is a liquid) was added slowly to the reaction mixture via a microliter syringe. The mixture was kept at $-45^{\circ} \mathrm{C}$ and stirred for $48 \mathrm{~h}$. After complete consumption of the allylboronate 3, TESCl (23 mg, $0.15 \mathrm{mmol}, 1.5$ equiv), imidazole (14 mg, $0.2 \mathrm{mmol}, 2.0$ equiv) and DMF $(0.2 \mathrm{ml})$ were added to the mixture. The reaction mixture was stirred at ambient temperature for additional $4 \mathrm{~h}$. Then diethyl ether $(1 \mathrm{~mL})$ and brine $(1 \mathrm{~mL})$ was added. The organic layer was separated and the aqueous layer was extracted with $\mathrm{Et}_{2} \mathrm{O}(2 \mathrm{~mL}$ x 3$)$. The combined organic extracts were dried over anhydrous sodium sulfate, filtered, and concentrated under reduced pressure. Purification of the crude product was performed by flash chromatography (gradient elution with hexane and ethyl acetate) to give product 5.

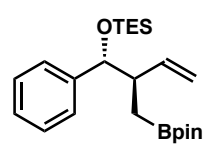

Triethyl(((1R,2R)-1-phenyl-2-((4,4,5,5-tetramethyl-1,3,2-dioxaborolan -2-yl)methyl)but-3-en-1-yl)oxy)silane (5a) Prepared according to the general procedure. The crude mixture was purified by column chromatography (hexane:ethyl acetate $=80: 1$ to $60: 1$ ) to give compound 5a in $84 \%$ yield (34 mg) as colorless oil. The enantiomeric excess was determined by HPLC analysis to be $99 \%$ ee $\left(254 \mathrm{~nm}, 25^{\circ} \mathrm{C}\right) ; \mathrm{t}_{1}=11.9 \mathrm{~min}, \mathrm{t}_{2}=13.0 \mathrm{~min}$ [(Chiralpak IA) hexane/i-PrOH, 99.6:0.4, $1.0 \mathrm{~mL} / \mathrm{min}] ;[\alpha]_{\mathrm{D}}{ }^{25}=3.14\left(\mathrm{c} 0.42, \mathrm{CHCl}_{3}\right)$.

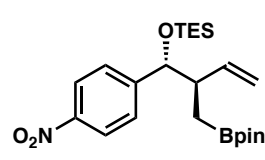

Triethyl(((1R,2R)-1-(4-nitrophenyl)-2-((4,4,5,5-tetramethyl-1,3,2-di oxaborolan-2-yl)methyl)but-3-en-1-yl)oxy)silane (5b) Prepared according to the general procedure. The crude mixture was purified by column chromatography (hexane:ethyl acetate $=80: 1$ to $60: 1$ ) to give compound $\mathbf{5 b}$ in $83 \%$ yield $(37 \mathrm{mg})$ as colorless oil. The enantiomeric excess of mono-TES protected diol (benzylic) was determined by HPLC analysis to be $99 \%$ ee $\left(254 \mathrm{~nm}, 25^{\circ} \mathrm{C}\right) ; \mathrm{t}_{1}=9.05$ $\min , \mathrm{t}_{2}=9.47 \mathrm{~min}\left[(\right.$ Chiralpak ID) hexane/i-PrOH, 98:2, $1.0 \mathrm{~mL} / \mathrm{min}] ;[\alpha]_{\mathrm{D}}{ }^{25}=0.06(\mathrm{c}$ $\left.0.33, \mathrm{CHCl}_{3}\right)$. 


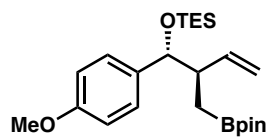

Triethyl(((1R,2R)-1-(4-methoxyphenyl)-2-((4,4,5,5-tetramethyl-1, 3, 2-dioxaborolan-2-yl)methyl)but-3-en-1-yl)oxy)silane (5c) Prepared according to the general procedure. The crude mixture was purified by column chromatography (hexane:ethyl acetate $=80: 1$ to $60: 1$ ) to give compound $\mathbf{5} \mathbf{c}$ in $81 \%$ yield (35 mg) as colorless oil. The enantiomeric excess of mono-TES protected diol (benzylic) was determined by HPLC analysis to be $99 \%$ ee $\left(254 \mathrm{~nm}, 25^{\circ} \mathrm{C}\right) ; \mathrm{t}_{1}=8.06$ $\min , \mathrm{t}_{2}=8.52 \mathrm{~min}\left[(\right.$ Chiralpak IA) hexane/i-PrOH, 98:2, $1.0 \mathrm{~mL} / \mathrm{min}] ;[\alpha]_{\mathrm{D}}{ }^{25}=0.83(\mathrm{c}$ $\left.0.80, \mathrm{CHCl}_{3}\right)$.

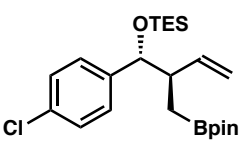
(((1R,2R)-1-(4-chlorophenyl)-2-((4,4,5,5-tetramethyl-1,3,2-dioxaboro lan-2-yl)methyl)but-3-en-1-yl)oxy)triethylsilane (5d) Prepared according to the general procedure. The crude mixture was purified by column chromatography (hexane:ethyl acetate $=80: 1$ to $60: 1$ ) to give compound $\mathbf{3 d}$ in $80 \%$ yield (35 mg) as colorless oil. The enantiomeric excess was determined by HPLC analysis to be $99 \%$ ee $\left(254 \mathrm{~nm}, 25^{\circ} \mathrm{C}\right) ; \mathrm{t}_{1}=15.1 \mathrm{~min}, \mathrm{t}_{2}=16.2 \mathrm{~min}$ [(Chiralpak IA) hexane/i-PrOH, 99.6:0.4, $1.0 \mathrm{~mL} / \mathrm{min}] ;[\alpha]_{\mathrm{D}}{ }^{25}=2.69\left(\mathrm{c} 0.87, \mathrm{CHCl}_{3}\right)$.<smiles>C=CC(C[18OH])C(OC)c1ccccc1Cl</smiles>
(((1R,2R)-1-(2-chlorophenyl)-2-((4,4,5,5-tetramethyl-1,3,2-dioxaborolan -2-yl)methyl)but-3-en-1-yl)oxy)triethylsilane (5e) Prepared according to the general procedure. The crude mixture was purified by column chromatography (hexane:ethyl acetate $=80: 1$ to $60: 1$ ) to give compound $\mathbf{5 e}$ in $71 \%$ yield (31 mg) as colorless oil. The enantiomeric excess of mono-TES protected diol (benzylic) was determined by HPLC analysis to be $90 \%$ ee $\left(254 \mathrm{~nm}, 25^{\circ} \mathrm{C}\right) ; \mathrm{t}_{1}=11.9 \mathrm{~min}, \mathrm{t}_{2}=13.0$ $\min \left[(\right.$ Chiralpak IA) hexane/i-PrOH, 99.6:0.4, $1.0 \mathrm{~mL} / \mathrm{min}] ;[\alpha]_{\mathrm{D}}{ }^{25}=1.98$ (c 1.38, $\left.\mathrm{CHCl}_{3}\right)$.

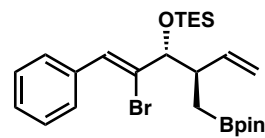

(((3R,4R,Z)-2-bromo-1-phenyl-4-((4,4,5,5-tetramethyl-1,3,2-dioxabo rolan-2-yl)methyl)hexa-1,5-dien-3-yl)oxy)triethylsilane (5f) Prepared according to the general procedure. The crude mixture was purified by column chromatography (hexane:ethyl acetate $=80: 1$ to $60: 1$ ) to give compound $\mathbf{5 f}$ in $87 \%$ yield (44 mg) as colorless oil. The enantiomeric excess of mono-TES protected diol (benzylic) was determined by HPLC analysis to be $99 \%$ ee $\left(254 \mathrm{~nm}, 25^{\circ} \mathrm{C}\right) ; \mathrm{t}_{1}=13.8$ $\min , \mathrm{t}_{2}=15.1 \mathrm{~min}\left[(\right.$ Chiralpak IA) hexane $/ \mathrm{i}-\mathrm{PrOH}, 99.6: 0.4,1.0 \mathrm{~mL} / \mathrm{min}] ;[\alpha]_{\mathrm{D}}{ }^{25}=2.16$ (c $1.23, \mathrm{CHCl}_{3}$ ). 


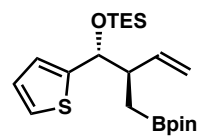

Triethyl(((1R,2R)-2-((4,4,5,5-tetramethyl-1,3,2-dioxaborolan-2-yl)meth yl)-1-(thiophen-2-yl)but-3-en-1-yl)oxy)silane (5g) Prepared according to

the general procedure. The crude mixture was purified by column chromatography (hexane:ethyl acetate $=80: 1$ to $60: 1$ ) to give compound $\mathbf{5 g}$ in 78\% yield $(32 \mathrm{mg}$ ) as colorless oil. The enantiomeric excess of mono-TES protected diol (benzylic) was determined by HPLC analysis to be $98 \%$ ee $\left(254 \mathrm{~nm}, 25^{\circ} \mathrm{C}\right) ; \mathrm{t}_{1}=15.9 \mathrm{~min}, \mathrm{t}_{2}=17.6$ $\min \left[(\right.$ Chiralpak IA) hexane/i-PrOH, 99.6:0.4, $1.0 \mathrm{~mL} / \mathrm{min}] ;[\alpha]_{\mathrm{D}}{ }^{25}=2.80$ (c 1.40, $\left.\mathrm{CHCl}_{3}\right)$.

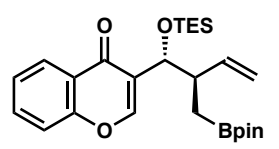

3-((1R,2R)-2-((4,4,5,5-tetramethyl-1,3,2-dioxaborolan-2-yl)methyl)1-((triethylsilyl)oxy)but-3-en-1-yl)-4H-chromen-4-one (5h) Prepared according to the general procedure. The crude mixture was purified by column chromatography (hexane:ethyl acetate $=80: 1$ to $60: 1$ ) to give compound $\mathbf{5 h}$ in $\mathbf{9 8 \%}$ yield $(46 \mathrm{mg})$ as colorless oil. The enantiomeric excess of mono-TES protected diol (benzylic) was determined by HPLC analysis to be $96 \%$ ee $\left(254 \mathrm{~nm}, 25{ }^{\circ} \mathrm{C}\right) ; \mathrm{t}_{1}=6.29$ $\min , \mathrm{t}_{2}=7.11 \mathrm{~min}\left[(\right.$ Chiralpak IC) hexane/i-PrOH, 98:2, $1.0 \mathrm{~mL} / \mathrm{min}] ;[\alpha]_{\mathrm{D}}{ }^{25}=5.63(\mathrm{c}$ $\left.1.62, \mathrm{CHCl}_{3}\right)$.

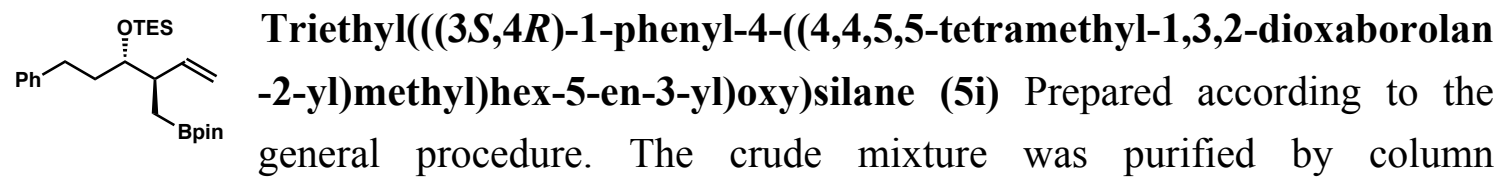
chromatography (hexane:ethyl acetate $=80: 1$ to $60: 1$ ) to give compound $\mathbf{5 i}$ in $86 \%$ yield (37 mg) as colorless oil. The enantiomeric excess of the corresponding diol was determined by HPLC analysis to be $93 \%$ ee $\left(254 \mathrm{~nm}, 25^{\circ} \mathrm{C}\right) ; \mathrm{t}_{1}=7.01 \mathrm{~min}, \mathrm{t}_{2}=7.62 \mathrm{~min}$ [(Chiralpak ID) hexane/i-PrOH, 90:10, $1.0 \mathrm{~mL} / \mathrm{min}] ;[\alpha]_{\mathrm{D}}{ }^{25}=-0.92\left(\mathrm{c} 0.87, \mathrm{CHCl}_{3}\right)$.

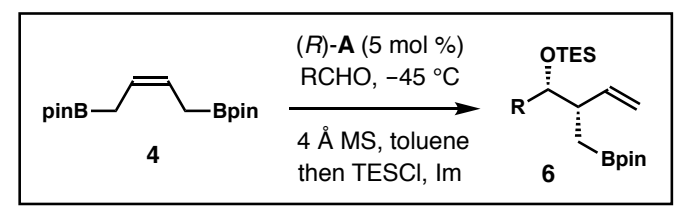

General procedure for synthesis of chiral TES-protected homoallylic alcohols 6: To a reaction flask containing a stir bar, freshly activated $4 \AA \mathrm{MS}(25 \mathrm{mg})$ and phosphoric acid $(R)$-A $(3.8 \mathrm{mg}, 0.005 \mathrm{mmol})$, allylboronate $4(0.1 \mathrm{mmol})$ and toluene $(0.3 \mathrm{~mL})$ was added to the flask. The mixture was placed in a $-45{ }^{\circ} \mathrm{C}$ cold bath and stirred for $5 \mathrm{~min}$. Then freshly distilled aldehyde ( $0.2 \mathrm{mmol}$, if it is a liquid) was added slowly to the reaction mixture via a microliter syringe. The mixture was kept at $-45{ }^{\circ} \mathrm{C}$ and stirred for 
$48 \mathrm{~h}$. After complete consumption of the allylboronate 4, TESCl (23 mg, $0.15 \mathrm{mmol}, 1.5$ equiv), imidazole (14 mg, $0.2 \mathrm{mmol}, 2.0$ equiv) and $\mathrm{DMF}(0.2 \mathrm{ml})$ were added to the mixture. The reaction mixture was stirred at ambient temperature for additional $4 \mathrm{~h}$. Then diethyl ether $(1 \mathrm{~mL})$ and brine $(1 \mathrm{~mL})$ was added. The organic layer was separated and the aqueous layer was extracted with $\mathrm{Et}_{2} \mathrm{O}(2 \mathrm{~mL} \times 3)$. The combined organic extracts were dried over anhydrous sodium sulfate, filtered, and concentrated under reduced pressure. Purification of the crude product was performed by flash chromatography (gradient elution with hexane and ethyl acetate) to give product $\mathbf{6}$.

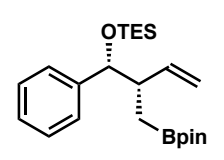

Triethyl(((1R,2S)-1-phenyl-2-((4,4,5,5-tetramethyl-1,3,2-dioxaborolan-2 -yl)methyl)but-3-en-1-yl)oxy)silane (6a) Prepared according to the general procedure. The crude mixture was purified by column chromatography (hexane:ethyl acetate $=80: 1$ to $60: 1)$ to give compound $\mathbf{6 a}$ in $82 \%$ yield $(33 \mathrm{mg}$ ) as colorless oil. The enantiomeric excess of the corresponding diol was determined by HPLC analysis to be $86 \%$ ee $\left(254 \mathrm{~nm}, 25^{\circ} \mathrm{C}\right) ; \mathrm{t}_{1}=17.6 \mathrm{~min}, \mathrm{t}_{2}=18.7 \mathrm{~min}$ [(Chiralpak IE) hexane/i-PrOH, 95:5, $1.0 \mathrm{~mL} / \mathrm{min}]$.

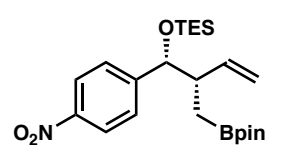

Triethyl(((1R,2S)-1-(4-nitrophenyl)-2-((4,4,5,5-tetramethyl-1,3,2-dio xaborolan-2-yl)methyl)but-3-en-1-yl)oxy)silane

(6b) Prepared

according to the general procedure. The crude mixture was purified (hexane:ethyl acetate $=80: 1$ to $60: 1)$ to give compound $\mathbf{6 b}$ in $78 \%$ yield $(35 \mathrm{mg}$ ) as colorless oil. The enantiomeric excess of the corresponding alcohol after oxidation was determined by HPLC analysis to be $86 \%$ ee $\left(254 \mathrm{~nm}, 25{ }^{\circ} \mathrm{C}\right) ; \mathrm{t}_{1}=13.4 \mathrm{~min}, \mathrm{t}_{2}=14.2 \mathrm{~min}$ [(Chiralpak IA) hexane/i-PrOH, 98:2, $1.0 \mathrm{~mL} / \mathrm{min}] ;[\alpha]_{\mathrm{D}}{ }^{25}=-0.10\left(\mathrm{c} 0.80, \mathrm{CHCl}_{3}\right)$.

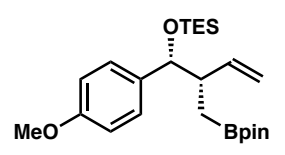

Triethyl(((1R,2S)-1-(4-methoxyphenyl)-2-((4,4,5,5-tetramethyl-1,3,2 -dioxaborolan-2-yl)methyl)but-3-en-1-yl)oxy)silane (6c) Prepared according to the general procedure. The crude mixture was purified by column chromatography (hexane:ethyl acetate $=80: 1$ to $60: 1$ ) to give compound $\mathbf{6 c}$ in $88 \%$ yield (38 mg) as colorless oil. The enantiomeric excess of the corresponding diol was determined by HPLC analysis to be $82 \%$ ee $\left(254 \mathrm{~nm}, 25^{\circ} \mathrm{C}\right) ; \mathrm{t}_{1}=15.9 \mathrm{~min}, \mathrm{t}_{2}=16.9 \mathrm{~min}$ [(Chiralpak IG) hexane/i-PrOH, 90:10, $1.0 \mathrm{~mL} / \mathrm{min}]$.

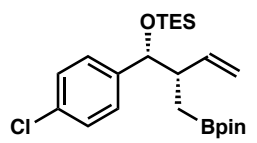

(((1R,2S)-1-(4-chlorophenyl)-2-((4,4,5,5-tetramethyl-1,3,2-dioxaborol an-2-yl)methyl)but-3-en-1-yl)oxy)triethylsilane (6d) Prepared according to the general procedure. The crude mixture was purified 
(hexane:ethyl acetate $=80: 1$ to $60: 1$ ) to give compound $\mathbf{6 d}$ in $78 \%$ yield $(34 \mathrm{mg}$ ) as colorless oil. The enantiomeric excess of mono-TES protected diol (benzylic) was determined by HPLC analysis to be $84 \%$ ee $\left(254 \mathrm{~nm}, 25^{\circ} \mathrm{C}\right) ; \mathrm{t}_{1}=12.0 \mathrm{~min}, \mathrm{t}_{2}=13.2 \mathrm{~min}$ [(Chiralpak IA) hexane/i-PrOH, 99.6:0.4, $1.0 \mathrm{~mL} / \mathrm{min}] ;[\alpha]_{\mathrm{D}}{ }^{25}=0.72\left(\mathrm{c} 0.74, \mathrm{CHCl}_{3}\right)$.

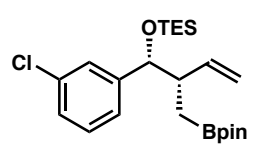

(((1R,2S)-1-(3-chlorophenyl)-2-((4,4,5,5-tetramethyl-1,3,2-dioxaborol an-2-yl)methyl)but-3-en-1-yl)oxy)triethylsilane

(6e) Prepared according to the general procedure. The crude mixture was purified by column chromatography (hexane:ethyl acetate $=80: 1$ to $60: 1$ ) to give compound $\mathbf{6 e}$ in $76 \%$ yield $(33 \mathrm{mg})$ as colorless oil. The enantiomeric excess of the corresponding diol was determined by HPLC analysis to be $87 \%$ ee $\left(254 \mathrm{~nm}, 25^{\circ} \mathrm{C}\right) ; \mathrm{t}_{1}=17.3 \mathrm{~min}, \mathrm{t}_{2}=22.6 \mathrm{~min}$ [(Chiralpak IA) hexane/i-PrOH, 98:2, $1.0 \mathrm{~mL} / \mathrm{min}]$.

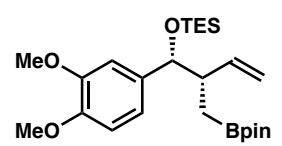

(((1R,2S)-1-(3,4-dimethoxyphenyl)-2-((4,4,5,5-tetramethyl-1,3,2-dio xaborolan-2-yl)methyl)but-3-en-1-yl)oxy)triethylsilane (6f) Prepared according to the general procedure. The crude mixture was purified by column chromatography (hexane:ethyl acetate $=80: 1$ to $60: 1$ ) to give compound $\mathbf{6} \mathbf{f}$ in $76 \%$ yield $(35 \mathrm{mg})$. The enantiomeric excess of the corresponding diol was determined by HPLC analysis to be $81 \%$ ee $\left(254 \mathrm{~nm}, 25^{\circ} \mathrm{C}\right) ; \mathrm{t}_{1}=25.4 \mathrm{~min}, \mathrm{t}_{2}=28.2 \mathrm{~min}$ [(Chiralpak IG) hexane/i-PrOH, 90:10, $1.0 \mathrm{~mL} / \mathrm{min}] ;{ }^{1} \mathrm{H}$ NMR $\left(500 \mathrm{MHz}, \mathrm{CDCl}_{3}\right) \delta 6.87(\mathrm{~s}, 1 \mathrm{H}), 6.74(\mathrm{~d}$, $J=8.4 \mathrm{~Hz}, 1 \mathrm{H}), 6.72(\mathrm{~d}, J=8.3 \mathrm{~Hz}, 1 \mathrm{H}), 5.63-5.70(\mathrm{~m}, 1 \mathrm{H}), 4.88-4.91(\mathrm{~m}, 2 \mathrm{H}), 4.44$ (d, $J=6.0 \mathrm{~Hz}, 1 \mathrm{H}), 3.87(\mathrm{~s}, 3 \mathrm{H}), 3.86(\mathrm{~s}, 3 \mathrm{H}), 2.53-2.58(\mathrm{~m}, 1 \mathrm{H}), 1.21(\mathrm{~s}, 6 \mathrm{H}), 1.20(\mathrm{~s}$, $6 \mathrm{H}), 1.08(\mathrm{dd}, J=15.3,4.6 \mathrm{~Hz}, 1 \mathrm{H}), 0.81-0.87(\mathrm{~m}, 10 \mathrm{H}), 0.42-0.54(\mathrm{~m}, 6 \mathrm{H}) .{ }^{13} \mathrm{C}$ NMR (126 MHz, $\left.\mathrm{CDCl}_{3}\right) \delta 148.6,148.1,141.0,136.9,119.6,115.0,110.4,110.3,83.3$, 79.0, 56.1(two overlapping carbon signals), 48.4, 25.3, 25.1, 7.2, 5.2. $\mathrm{HRMS}\left(\mathrm{ESI}^{+}\right): \mathrm{m} / \mathrm{z}$ for $\mathrm{C}_{25} \mathrm{H}_{43} \mathrm{BO}_{5} \mathrm{NaSi}[\mathrm{M}+\mathrm{Na}]^{+}$calcd. 485.2871, found: 485.2868 .

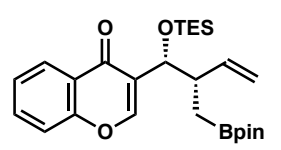

\section{3-((1R,2S)-2-((4,4,5,5-tetramethyl-1,3,2-dioxaborolan-2-yl)methyl)-1} -((triethylsilyl)oxy)but-3-en-1-yl)-4H-chromen-4-one (6g) Prepared according to the general procedure. The crude mixture was purified by column chromatography (hexane:ethyl acetate $=80: 1$ to $60: 1$ ) to give compound $6 \mathbf{g}$ in $62 \%$ yield $(29 \mathrm{mg})$ as colorless oil. The enantiomeric excess of the corresponding alcohol after oxidation was determined by HPLC analysis to be $91 \%$ ee $\left(254 \mathrm{~nm}, 25^{\circ} \mathrm{C}\right) ; \mathrm{t}_{1}=17.9 \mathrm{~min}$, $\mathrm{t}_{2}=19.9 \mathrm{~min}\left[(\right.$ Chiralpak IA) hexane/i-PrOH, $98: 2,1.0 \mathrm{~mL} / \mathrm{min}] ;[\alpha]_{\mathrm{D}}{ }^{25}=-2.95(\mathrm{c} 0.86$, $\left.\mathrm{CHCl}_{3}\right)$. 


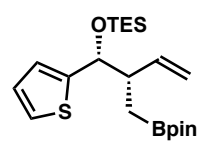

Triethyl(((1R,2S)-2-((4,4,5,5-tetramethyl-1,3,2-dioxaborolan-2-yl)methy l)-1-(thiophen-2-yl)but-3-en-1-yl)oxy)silane (6h) Prepared according to

the general procedure. The crude mixture was purified by column chromatography (hexane:ethyl acetate $=80: 1$ to $60: 1$ ) to give compound $\mathbf{6 h}$ in $86 \%$ yield (35 mg) as colorless oil. The enantiomeric excess of the corresponding diol was determined by HPLC analysis to be $70 \%$ ee $\left(254 \mathrm{~nm}, 25^{\circ} \mathrm{C}\right) ; \mathrm{t}_{1}=10.5 \mathrm{~min}, \mathrm{t}_{2}=11.2 \mathrm{~min}$ [(Chiralpak IG) hexane/i-PrOH, 90:10, $1.0 \mathrm{~mL} / \mathrm{min}]$.

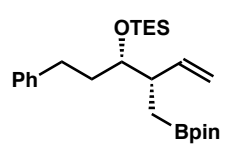

Triethyl(((3S,4S)-1-phenyl-4-((4,4,5,5-tetramethyl-1,3,2-dioxaborolan2-yl)methyl)hex-5-en-3-yl)oxy)silane (6i) Prepared according to the general procedure. The crude mixture was purified by column chromatography (hexane:ethyl acetate $=80: 1$ to $60: 1$ ) to give compound $6 \mathbf{i}$ in $79 \%$ yield (34 mg) as colorless oil. The enantiomeric excess of the corresponding diol was determined by HPLC analysis to be $74 \%$ ee $\left(254 \mathrm{~nm}, 25^{\circ} \mathrm{C}\right) ; \mathrm{t}_{1}=7.00 \mathrm{~min}, \mathrm{t}_{2}=7.60 \mathrm{~min}$ [(Chiralpak IC) hexane/i-PrOH, 90:10, $1.0 \mathrm{~mL} / \mathrm{min}]$.

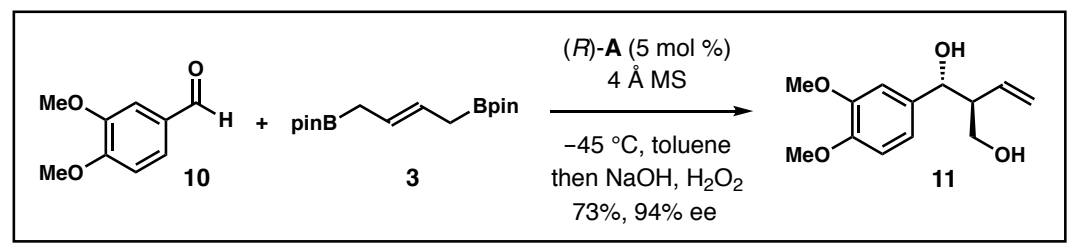

(1R,2S)-1-(3,4-dimethoxyphenyl)-2-vinylpropane-1,3-diol (11) To a reaction flask containing a stir bar, freshly activated $4 \AA \mathrm{MS}(25 \mathrm{mg})$ and phosphoric acid $(R)$-A (3.8 $\mathrm{mg}, 0.005 \mathrm{mmol})$, allylboronate $3(246 \mathrm{mg}, 0.80 \mathrm{mmol})$ and toluene $(2 \mathrm{~mL})$ was added to the flask. The mixture was placed in a $-45{ }^{\circ} \mathrm{C}$ cold bath and stirred for 5 min. Then veratraldehyde $(1.6 \mathrm{mmol}, 264 \mathrm{mg})$ was added slowly to the reaction mixture via a microliter syringe. The mixture was kept at $-45^{\circ} \mathrm{C}$ and stirred for $48 \mathrm{~h}$. After complete consumption of the allylboronate $3, \mathrm{NaOH}(3 \mathrm{~N}$ aqueous, $3 \mathrm{~mL}$ ) was added to the reaction mixture followed by slow addition of $30 \% \mathrm{H}_{2} \mathrm{O}_{2}(3 \mathrm{~mL})$ at $0{ }^{\circ} \mathrm{C}$. The reaction was stirred vigorously for $6 \mathrm{~h}$; then EtOAc $(5 \mathrm{~mL})$ and brine $(5 \mathrm{~mL})$ was added. The organic layer was separated and the aqueous layer was extracted with EtOAc $(5 \mathrm{~mL} \times 3)$. The combined organic extracts were dried over anhydrous sodium sulfate, filtered, and concentrated under reduced pressure. Purification of the crude product was performed by flash chromatography (gradient elution with hexane and ethyl acetate 2:1 to 1:1) to give product diol 11 in $73 \%$ yield $(139 \mathrm{mg})$. A 1-mmol-scale reaction was also conducted to give product 11 in $78 \%$ yield $(186 \mathrm{mg})$. The enantiomeric excess of the corresponding 
mono-TES protected diol (benzylic) was determined by HPLC analysis to be $94 \%$ ee $\left(254 \mathrm{~nm}, 25^{\circ} \mathrm{C}\right) ; \mathrm{t}_{1}=16.6 \mathrm{~min}, \mathrm{t}_{2}=17.7 \mathrm{~min}$ [(Chiralpak IA) hexane/i-PrOH, 98:2, 1.0 $\mathrm{mL} / \mathrm{min}] ;{ }^{1} \mathrm{H}$ NMR $\left(600 \mathrm{MHz}, \mathrm{CDCl}_{3}\right) \delta 6.91(\mathrm{~d}, J=1.7 \mathrm{~Hz}, 1 \mathrm{H}), 6.87(\mathrm{dd}, J=8.2,1.8$ $\mathrm{Hz}, 1 \mathrm{H}), 6.84$ (d, $J=8.2 \mathrm{~Hz}, 1 \mathrm{H}), 5.84$ (ddd, $J=17.4,10.3,8.8 \mathrm{~Hz}, 1 \mathrm{H}), 5.30$ (dd, $J=$ 10.4, $1.5 \mathrm{~Hz}, 1 \mathrm{H}), 5.22(\mathrm{~d}, J=17.3 \mathrm{~Hz}, 1 \mathrm{H}), 4.75(\mathrm{~d}, J=6.3 \mathrm{~Hz}, 1 \mathrm{H}), 3.89(\mathrm{~s}, 3 \mathrm{H}), 3.88$ (s, 3H), 3.63 (dd, $J=10.7,5.5 \mathrm{~Hz}, 1 \mathrm{H}), 3.58$ (dd, $J=10.7,6.2 \mathrm{~Hz}, 1 \mathrm{H}), 2.57-2.61$ (m, 1H), 2.37 (bs, 1H), 1.58 (bs, 1H). ${ }^{13} \mathrm{C}$ NMR (126 MHz, $\left.\mathrm{CDCl}_{3}\right) \delta 149.4,148.9,136.0$, 135.0, 120.1, 119.1, 111.2, 109.8, 75.2, 64.1, 56.3, 56.2, 54.1. HRMS $\left(\mathrm{ESI}^{+}\right): \mathrm{m} / \mathrm{z}$ for $\mathrm{C}_{13} \mathrm{H}_{18} \mathrm{O}_{4} \mathrm{Na}[\mathrm{M}+\mathrm{Na}]^{+}$calcd. 261.1103, found: 261.1104.

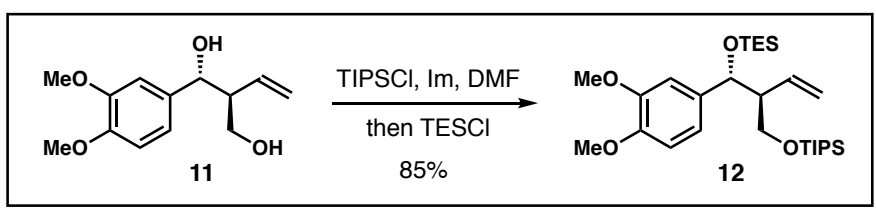

(6S,7R)-7-(3,4-dimethoxyphenyl)-9,9-diethyl-3,3-diisopropyl-2-methyl-6-vinyl-4,8-di oxa-3,9-disilaundecane 12: To a reaction vial containing a Teflon-coated magnetic stirring bar were added diol 11 (119 mg, $0.50 \mathrm{mmol}, 1.0$ equiv) and DMF (0.7 mL) followed by addition of TIPSCl (192 mg, $1.0 \mathrm{mmol}, 2.0$ equiv) and imidazole ( $88 \mathrm{mg}$, $1.25 \mathrm{mmol}, 2.5$ equiv). The reaction mixture was then allowed to stir at ambient temperature for $2 \mathrm{~h}$. Then TESCl $(151 \mathrm{mg}, 1.0 \mathrm{mmol}, 2.0$ equiv) and imidazole $(88 \mathrm{mg}$, $1.25 \mathrm{mmol}, 2.5$ equiv) were added to the reaction mixture. The reaction was stirred for 1 h. Then diethyl ether $(1 \mathrm{~mL})$ and brine $(1 \mathrm{~mL})$ was added. The organic layer was separated and the aqueous layer was extracted with ethyl ether $(2 \mathrm{~mL} \times 3)$. The combined organic extracts were dried over anhydrous sodium sulfate, filtered, and concentrated under reduced pressure. Purification of the crude product was performed by flash chromatography (gradient elution with hexane and ethyl acetate 50:1 to 30:1) to give product TES-protected homoallylic alcohol 12 in $85 \%$ yield $\left(216 \mathrm{mg}\right.$ ). ${ }^{1} \mathrm{H}$ NMR (500 $\left.\mathrm{MHz}, \mathrm{CDCl}_{3}\right) \delta 6.86(\mathrm{~s}, 1 \mathrm{H}), 6.77$ (app.s, 2H), 5.87 (ddd, $\left.J=17.9,9.6,9.6 \mathrm{~Hz}, 1 \mathrm{H}\right), 5.03$ (d, $J=10.4 \mathrm{~Hz}, 1 \mathrm{H}), 4.98-4.99(\mathrm{~m}, 1 \mathrm{H}), 4.90$ (d, $J=17.4 \mathrm{~Hz}, 1 \mathrm{H}), 3.863(\mathrm{~s}, 3 \mathrm{H}), 3.859$ (s, 3H), 3.77 (dd, $J=8.4,8.4 \mathrm{~Hz}, 1 \mathrm{H}), 3.51$ (dd, $J=9.3,5.2 \mathrm{~Hz}, 1 \mathrm{H}), 2.29-2.34$ (m, 1H), $1.07-1.13(\mathrm{~m}, 21 \mathrm{H}), 0.87(\mathrm{t}, J=7.9 \mathrm{~Hz}, 9 \mathrm{H}), 0.51(\mathrm{q}, J=8.0 \mathrm{~Hz}, 6 \mathrm{H}) \cdot{ }^{13} \mathrm{C}$ NMR $(126$ $\left.\mathrm{MHz}, \mathrm{CDCl}_{3}\right) \delta 148.6,148.0,137.6,136.4,118.9,117.7,110.6,110.1,73.4,64.6,56.5$, 56.13, 56.05, 18.4, 12.4, 7.2, 5.2. HRMS $\left(\mathrm{ESI}^{+}\right): \mathrm{m} / \mathrm{z}$ for $\mathrm{C}_{28} \mathrm{H}_{52} \mathrm{O}_{4} \mathrm{NaSi}_{2}[\mathrm{M}+\mathrm{Na}]^{+}$calcd. 531.3302, found: 531.3279 . 


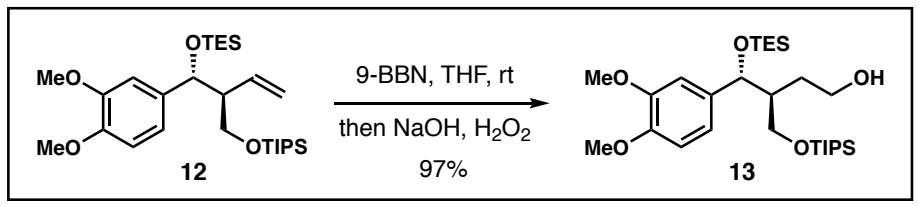

(3S,4R)-4-(3,4-dimethoxyphenyl)-4-((triethylsilyl)oxy)-3-(((triisopropylsilyl)oxy)

methyl)butan-1-ol 13: In an Ar-filled glove box, 12 (203 mg, $0.40 \mathrm{mmol}, 1.0$ equiv), THF (0.7 mL), 9-BBN (73 mg, $0.60 \mathrm{mmol}, 1.5$ equiv) and a Teflon-coated magnetic stir bar were sequentially added to a 1-dram vial. And the mixture was stirred for $2 \mathrm{~h}$ at ambient temperature in the glove box. After complete consumption of 12, the vial was removed from glove box. Then $\mathrm{NaOH}$ ( $3 \mathrm{~N}$ aqueous, $0.5 \mathrm{~mL}$ ) was added to the reaction mixture followed by slow addition of $30 \% \mathrm{H}_{2} \mathrm{O}_{2}(0.5 \mathrm{~mL})$ at ambient temperature for $1 \mathrm{~h}$. Then diethyl ether $(1 \mathrm{~mL})$ and brine $(1 \mathrm{~mL})$ was added. The organic layer was separated and the aqueous layer was extracted with $\mathrm{Et}_{2} \mathrm{O}(2 \mathrm{~mL} \times 3)$. The combined organic extracts were dried over anhydrous sodium sulfate, filtered, and concentrated under reduced pressure. Purification of the crude product was performed by flash chromatography (gradient elution with hexane and ethyl acetate 20:1 to 10:1) to give product TES-protected homoallylic alcohol 13 in 97\% yield (204 mg). ${ }^{1} \mathrm{H}$ NMR (500 $\left.\mathrm{MHz}, \mathrm{CDCl}_{3}\right) \delta 6.87(\mathrm{~d}, J=1.4 \mathrm{~Hz}, 1 \mathrm{H}), 6.77-6.78(\mathrm{~m}, 2 \mathrm{H}), 4.70(\mathrm{~d}, J=5.5 \mathrm{~Hz}, 1 \mathrm{H})$, $3.873(\mathrm{~s}, 3 \mathrm{H}), 3.868(\mathrm{~s}, 3 \mathrm{H}), 3.74(\mathrm{dd}, J=10.0,4.7 \mathrm{~Hz}, 1 \mathrm{H}), 3.62(\mathrm{t}, J=5.6 \mathrm{~Hz}, 2 \mathrm{H}), 3.47$ $(\mathrm{dd}, J=9.9,7.0 \mathrm{~Hz}, 1 \mathrm{H}), 3.18(\mathrm{bs}, 1 \mathrm{H}), 1.85-1.93(\mathrm{~m}, 2 \mathrm{H}), 1.55-1.65(\mathrm{~m}, 1 \mathrm{H}), 0.98-$ $1.11(\mathrm{~m}, 21 \mathrm{H}), 0.87(\mathrm{t}, J=7.9 \mathrm{~Hz}, 9 \mathrm{H}), 0.45-0.58(\mathrm{~m}, 6 \mathrm{H}) .{ }^{13} \mathrm{C} \mathrm{NMR}\left(126 \mathrm{MHz}, \mathrm{CDCl}_{3}\right)$ $\delta 149.0,148.4,136.7,119.1,110.8,109.8,75.5,65.2,61.9,56.2,56.1,49.0,32.0,18.3$, 12.3, 7.1, 5.1. HRMS (ESI $\left.{ }^{+}\right): \mathrm{m} / \mathrm{z}$ for $\mathrm{C}_{28} \mathrm{H}_{54} \mathrm{O}_{5} \mathrm{Si}_{2} \mathrm{Na}[\mathrm{M}+\mathrm{Na}]^{+}$calcd. 549.3408, found: 549.3420 .

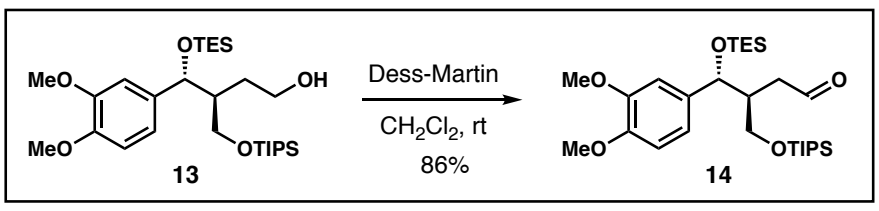

(3S,4R)-4-(3,4-dimethoxyphenyl)-4-((triethylsilyl)oxy)-3-(((triisopropylsilyl)oxy)

methyl)butanal 14: To a reaction vial containing a Teflon-coated magnetic stirring bar were added alcohol 13 (158 mg, $0.30 \mathrm{mmol}, 1.0$ equiv) and DCM (1.0 mL) followed by addition of DMP (191 mg, $0.45 \mathrm{mmol}, 1.5$ equiv). The reaction mixture was then allowed to stir at ambient temperature for $2 \mathrm{~h}$. After complete consumption of $\mathbf{1 3}$, the reaction mixture was diluted with diethyl ether $(1 \mathrm{~mL})$ and filtered through a pad of silica gel. After concentrated under reduced pressure, purification of the crude product was 
performed by flash chromatography (gradient elution with hexane and ethyl acetate 30:1 to $10: 1)$ to give aldehyde 14 in $86 \%$ yield $(135 \mathrm{mg}) .{ }^{1} \mathrm{H}$ NMR (600 $\left.\mathrm{MHz}, \mathrm{CDCl}_{3}\right) \delta 9.71$ (t, $J=2.2 \mathrm{~Hz}, 1 \mathrm{H}), 6.86(\mathrm{~s}, 1 \mathrm{H}), 6.78-6.80(\mathrm{~m}, 2 \mathrm{H}), 4.75(\mathrm{~d}, J=6.4 \mathrm{~Hz}, 1 \mathrm{H}), 3.88(\mathrm{~s}$, $6 \mathrm{H}$ ), 3.63 (dd, $J=9.9,5.2 \mathrm{~Hz}, 1 \mathrm{H}$ ), 3.47 (dd, $J=9.9,6.1 \mathrm{~Hz}, 1 \mathrm{H}$ ), 2.61 (ddd, $J=16.7$, $5.7,2.1 \mathrm{~Hz}, 1 \mathrm{H}), 2.48$ (ddd, $J=16.7,7.0,2.3 \mathrm{~Hz}, 1 \mathrm{H}), 2.36-2.41(\mathrm{~m}, 1 \mathrm{H}), 1.01-1.07$ $(\mathrm{m}, 21 \mathrm{H}), 0.86(\mathrm{t}, J=7.9 \mathrm{~Hz}, 9 \mathrm{H}), 0.42-0.53(\mathrm{~m}, 6 \mathrm{H}) .{ }^{13} \mathrm{C} \mathrm{NMR}\left(126 \mathrm{MHz}, \mathrm{CDCl}_{3}\right) \delta$ 203.1, 149.2, 148.6, 136.5, 119.2, 110.9, 109.7, 74.5, 63.9, 56.2, 56.1, 46.7, 42.8, 18.3, 12.3, 7.1, 5.1. HRMS $\left(\mathrm{ESI}^{+}\right): \mathrm{m} / \mathrm{z}$ for $\mathrm{C}_{28} \mathrm{H}_{52} \mathrm{O}_{5} \mathrm{Si}_{2} \mathrm{Na}[\mathrm{M}+\mathrm{Na}]^{+}$calcd. 547.3251, found: 547.3243 .

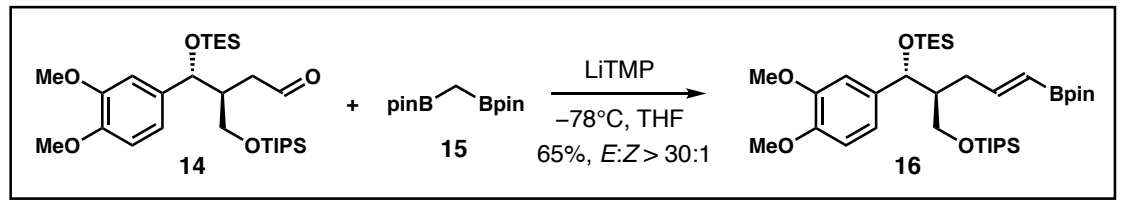

(6S,7R)-7-(3,4-dimethoxyphenyl)-9,9-diethyl-3,3-diisopropyl-2-methyl-6-((E)-3-(4,4,5 ,5-tetramethyl-1,3,2-dioxaborolan-2-yl)allyl)-4,8-dioxa-3,9-disilaundecane 16: A Teflon-coated magnetic stirring bar, LiTMP (88 mg, $0.60 \mathrm{mmol}, 2.0$ equiv) and THF (1.0 $\mathrm{mL}$ ) were added to an Ar-filled reaction vial. The reaction mixture was then allowed to stir at $0{ }^{\circ} \mathrm{C}$ for $5 \mathrm{~min}$. Then 1,1-diborylmethane (15, $161 \mathrm{mg}, 0.60 \mathrm{mmol}, 2.0$ equiv) was added to the mixture and stirred at $0{ }^{\circ} \mathrm{C}$ for $5 \mathrm{~min}$. Then reaction mixture was cooled to $-78{ }^{\circ} \mathrm{C}$ and aldehyde 14 (157 $\mathrm{mg}, 0.30 \mathrm{mmol}, 1.0$ equiv) was added. The reaction mixture was stirred at $-78{ }^{\circ} \mathrm{C}$ for $4 \mathrm{~h}$. After complete consumption of aldehyde 14 , the reaction mixture was quenched with sat. $\mathrm{NH}_{4} \mathrm{Cl}$ aqueous and extracted with $\mathrm{Et}_{2} \mathrm{O}(2 \mathrm{~mL} \mathrm{x}$ $3)$. The combined organic extracts were dried over anhydrous sodium sulfate, filtered, and concentrated under reduced pressure. Purification of the crude product was performed by flash chromatography (gradient elution with hexane and ethyl acetate 30:1 to $10: 1)$ to give vinylboronate 16 in $65 \%$ yield $(126 \mathrm{mg}) .{ }^{1} \mathrm{H} \mathrm{NMR}\left(600 \mathrm{MHz}, \mathrm{CDCl}_{3}\right) \delta$ $6.87(\mathrm{~s}, 1 \mathrm{H}), 6.79(\mathrm{~d}, J=7.6 \mathrm{~Hz}, 1 \mathrm{H}), 6.77$ (d, $J=7.9 \mathrm{~Hz}, 1 \mathrm{H}), 6.58$ (ddd, $J=17.5,6.8$, $6.8 \mathrm{~Hz}, 1 \mathrm{H}), 5.40(\mathrm{~d}, J=17.9 \mathrm{~Hz}, 1 \mathrm{H}), 4.89$ (d, $J=4.6 \mathrm{~Hz}, 1 \mathrm{H}), 3.863$ (s, 3H), 3.857 (s, $3 \mathrm{H}), 3.63-3.65(\mathrm{~m}, 1 \mathrm{H}), 3.41(\mathrm{dd}, J=9.9,4.3 \mathrm{~Hz}, 1 \mathrm{H}), 2.35-2.39(\mathrm{~m}, 1 \mathrm{H}), 2.16-2.22$ $(\mathrm{m}, 1 \mathrm{H}), 1.79-1.84(\mathrm{~m}, 1 \mathrm{H}), 1.24(\mathrm{~s}, 12 \mathrm{H}), 0.94-1.11(\mathrm{~m}, 21 \mathrm{H}), 0.87(\mathrm{t}, J=7.9 \mathrm{~Hz}$, 9H), 0.51 (q, $J=8.2 \mathrm{~Hz}, 6 \mathrm{H}) .{ }^{13} \mathrm{C}$ NMR $\left(126 \mathrm{MHz}, \mathrm{CDCl}_{3}\right) \delta 154.7,148.8,148.1,137.4$, $119.0,110.7,109.9,83.2,73.6,62.8,56.2,56.0,50.3,32.7,25.1,18.4,12.4,7.2,5.2$. HRMS (ESI ${ }^{+}$): $\mathrm{m} / \mathrm{z}$ for $\mathrm{C}_{35} \mathrm{H}_{65} \mathrm{O}_{6} \mathrm{BSi}_{2} \mathrm{Na}[\mathrm{M}+\mathrm{Na}]^{+}$calcd. 671.4310 , found: 671.4327. 


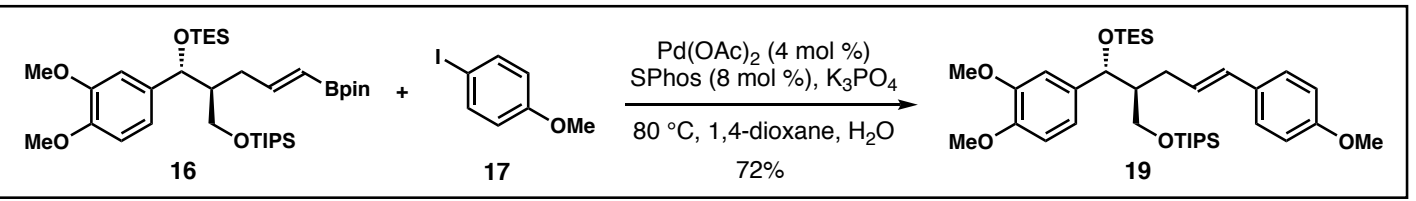

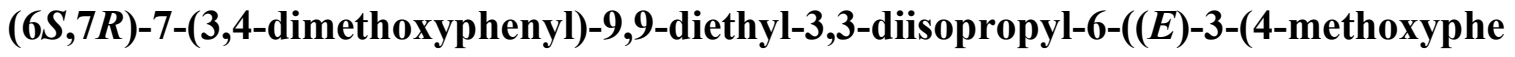
nyl)allyl)-2-methyl-4,8-dioxa-3,9-disilaundecane 19: A Teflon-coated magnetic stirring bar, $\mathrm{Pd}(\mathrm{OAc})_{2}(2 \mathrm{mg}, 0.008 \mathrm{mmol}, 4 \mathrm{~mol} \%)$, SPhos (7 mg, $0.016 \mathrm{mmol}, 8 \mathrm{~mol} \%$ ), $\mathrm{K}_{3} \mathrm{PO}_{4}(64 \mathrm{mg}, 0.60 \mathrm{mmol}, 3.0$ equiv) and 1,4-dioxane $(0.7 \mathrm{~mL})$ were added to an Ar-filled reaction vial. Then vinylboronate 16 (130 $\mathrm{mg}, 0.2 \mathrm{mmol}, 1.0$ equiv), 4-iodoanisole (51 mg, $0.22 \mathrm{mmol}, 1.1$ equiv) and $\mathrm{H}_{2} \mathrm{O}(6 \mu \mathrm{L})$ were added to the reaction vial. Then reaction mixture was heated to $80^{\circ} \mathrm{C}$ using heating mantle and stirred for $12 \mathrm{~h}$. After complete consumption of vinylboronate 16, the reaction mixture was diluted with diethyl ether $(1 \mathrm{~mL})$ and filtered through a pad of silica gel. After concentrated under reduced pressure, purification of the crude product was performed by flash chromatography (gradient elution with hexane and ethyl acetate 30:1 to 10:1) to give aldehyde 19 in $72 \%$ yield $(91 \mathrm{mg}) .{ }^{1} \mathrm{H}$ NMR $\left(500 \mathrm{MHz}, \mathrm{CDCl}_{3}\right) \delta 7.21(\mathrm{~d}, J=8.5 \mathrm{~Hz}$, 2H), $6.90(\mathrm{~s}, 1 \mathrm{H}), 6.79-6.83(\mathrm{~m}, 4 \mathrm{H}), 6.30(\mathrm{~d}, J=15.8 \mathrm{~Hz}, 1 \mathrm{H}), 6.01$ (ddd, $J=15.4,7.3$, $7.3 \mathrm{~Hz}, 1 \mathrm{H}), 4.90$ (d, J=5.5 Hz, 1H), $3.871(\mathrm{~s}, 3 \mathrm{H}), 3.866$ (s, 3H), 3.79 (s, 3H), 3.65 (dd, $J=9.8,6.3 \mathrm{~Hz}, 1 \mathrm{H}), 3.46(\mathrm{dd}, J=10.0,4.8 \mathrm{~Hz}, 1 \mathrm{H}), 2.41-2.46(\mathrm{~m}, 1 \mathrm{H}), 2.24-2.30(\mathrm{~m}$, 1H), $1.79-1.85(\mathrm{~m}, 1 \mathrm{H}), 0.94-1.03(\mathrm{~m}, 21 \mathrm{H}), 0.89$ (t, J= $7.9 \mathrm{~Hz}, 9 \mathrm{H}), 0.48-0.58(\mathrm{~m}$, $6 \mathrm{H}) .{ }^{13} \mathrm{C} \mathrm{NMR}\left(126 \mathrm{MHz}, \mathrm{CDCl}_{3}\right) \delta 158.9,148.9,148.1,137.6,131.2,130.7,128.2$, 127.2 , 119.1, 114.2, 110.8, 109.9, 73.8, 62.7, 56.2, 56.1, 55.6, 51.0, 29.7, 18.4, 12.4, 7.3, 5.2. HRMS (ESI $\left.{ }^{+}\right): \mathrm{m} / \mathrm{z}$ for $\mathrm{C}_{36} \mathrm{H}_{60} \mathrm{O}_{5} \mathrm{Si}_{2} \mathrm{Na}[\mathrm{M}+\mathrm{Na}]^{+}$calcd. 651.3877 , found: 651.3896 .

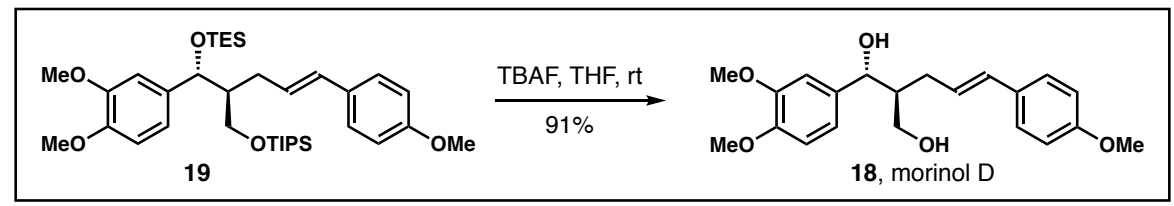

(1R,2S)-1-(3,4-dimethoxyphenyl)-2-((E)-3-(4-methoxyphenyl)allyl)propane-1,3-diol

(18, morinol D): A Teflon-coated magnetic stirring bar, compound 19 (31 mg, 0.05 mmol, 1.0 equiv $), \mathrm{THF}(0.5 \mathrm{~mL})$ and $\mathrm{TBAF}^{\cdot} \mathrm{H}_{2} \mathrm{O}(65 \mathrm{mg}, 0.25 \mathrm{mmol}, 5.0$ equiv) were added to a reaction vial. Then reaction mixture was stirred at ambient temperature for $12 \mathrm{~h}$. After complete consumption of compound 19, ethyl acetate $(1 \mathrm{~mL})$ and brine $(1 \mathrm{~mL})$ was added. The organic layer was separated and the aqueous layer was extracted with ethyl acetate (2 $\mathrm{mL} \times 3$ ). The combined organic extracts were dried over anhydrous sodium sulfate, filtered, and concentrated under reduced pressure. Purification of the crude product was 
performed by flash chromatography (gradient elution with hexane and ethyl acetate 2:1 to 1:1) to give morinol D (18) in 91\% yield (16 mg). ${ }^{1} \mathrm{H}$ NMR (500 MHz, $\left.\mathrm{CDCl}_{3}\right) \delta 7.24(\mathrm{~d}$, $J=8.6 \mathrm{~Hz}, 2 \mathrm{H}$ ), 6.95 (app. s, 1H), 6.91 (d, $J=8.3 \mathrm{~Hz}, 1 \mathrm{H}), 6.87$ (d, $J=8.2 \mathrm{~Hz}, 1 \mathrm{H}), 6.83$ (d, $J=8.4 \mathrm{~Hz}, 2 \mathrm{H}), 6.35$ (d, $J=15.7 \mathrm{~Hz}, 1 \mathrm{H}), 6.02$ (ddd, $J=15.3,7.3,7.3 \mathrm{~Hz}, 1 \mathrm{H}), 4.98$ (d, $J=4.0 \mathrm{~Hz}, 1 \mathrm{H}), 3.90(\mathrm{~s}, 3 \mathrm{H}), 3.89(\mathrm{~s}, 3 \mathrm{H}), 3.80(\mathrm{~s}, 3 \mathrm{H}), 3.71-3.77(\mathrm{~m}, 2 \mathrm{H}), 2.68(\mathrm{~d}$, $J=3.0 \mathrm{~Hz}, 1 \mathrm{H}$ ), 2.29 (dd, $J=7.1,7.1 \mathrm{~Hz} 2 \mathrm{H}), 2.03-2.09$ (m, 1H), 1.97 (t, $J=5.2 \mathrm{~Hz}$, $1 \mathrm{H}) .{ }^{13} \mathrm{C}$ NMR $\left(126 \mathrm{MHz}, \mathrm{CDCl}_{3}\right) \delta 159.2,149.3,148.7,135.4,131.5,130.6,127.4$, 126.7, 118.8, 114.3, 111.3, 109.7, 76.7, 64.3, 56.29, 56.27, 55.6, 47.4, 29.7. HRMS $\left(\mathrm{ESI}^{+}\right): \mathrm{m} / \mathrm{z}$ for $\mathrm{C}_{21} \mathrm{H}_{26} \mathrm{O}_{5} \mathrm{Na}[\mathrm{M}+\mathrm{Na}]^{+}$calcd. 381.1678 , found: 381.1677 .

\section{Preparation of acetonides S1 and S2 and determination of relative stereochemistry of 8 and 9:}

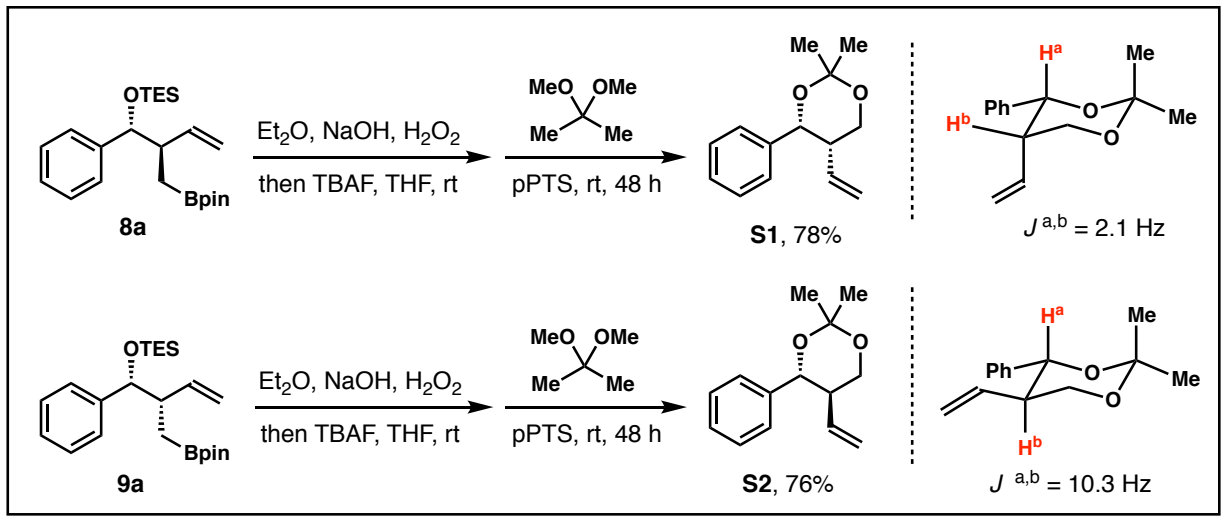

To a solution of TES-protected homoallylic alcohol (8a or 9a, 0.1 mmol) in $\mathrm{Et}_{2} \mathrm{O}, \mathrm{NaOH}$ ( $3 \mathrm{~N}$ aqueous, $0.5 \mathrm{~mL}$ ) was added to the reaction mixture followed by slow addition of $30 \%$ $\mathrm{H}_{2} \mathrm{O}_{2}(0.5 \mathrm{~mL})$ at $0{ }^{\circ} \mathrm{C}$. The reaction was stirred vigorously for $6 \mathrm{~h}$ at ambient temperature; then diethyl ether $(1 \mathrm{~mL})$ and brine $(1 \mathrm{~mL})$ was added. The organic layer was separated and the aqueous layer was extracted with $\mathrm{Et}_{2} \mathrm{O}(2 \mathrm{~mL} \times 3)$. The combined organic extracts were concentrated under reduced pressure. Then reaction mixture was dissolved in THF $(0.5 \mathrm{ml})$ and transferred to a reaction vial. A Teflon-coated magnetic stirring bar and TBAF $\mathrm{H}_{2} \mathrm{O}$ (65 mg, $0.25 \mathrm{mmol}, 2.5$ equiv) were added to the reaction vial. Then reaction mixture was stirred at ambient temperature for $2 \mathrm{~h}$. After complete consumption of the primary alcohol intermediate, ethyl acetate $(1 \mathrm{~mL})$ and brine $(1 \mathrm{~mL})$ was added. The organic layer was separated and the aqueous layer was extracted with ethyl acetate (2 $\mathrm{mL} \times 3$ ). The combined organic extracts were dried over anhydrous sodium sulfate, 
filtered, and concentrated under reduced pressure. Purification of the crude product was performed by flash chromatography (gradient elution with hexane and ethyl acetate) to give diol product. The diol product in 2, 2-dimethoxypropane $(1 \mathrm{~mL})$ was added pPTS $(0.01 \mathrm{mmol})$ and acetone $(0.1 \mathrm{~mL})$. The resulting mixture was stirred at ambient temperature for $48 \mathrm{~h}$. The mixture was filtered through a pad of silica gel and the solution was concentrated under reduced pressure. Purification of the crude product was performed by flash chromatography to give product $\mathbf{S 1}$ or $\mathbf{S 2}$ as colorless oil.

(4R,5S)-2,2-dimethyl-4-phenyl-5-vinyl-1,3-dioxane (S1) ${ }^{1} \mathrm{H}$ NMR $(400$
$\left.\mathrm{MHz}, \mathrm{CDCl}_{3}\right) \delta 7.21-7.51(\mathrm{~m}, 5 \mathrm{H}), 6.05(\mathrm{ddd}, J=17.7,10.7 \mathrm{~Hz}, 9.7 \mathrm{~Hz}$, 1H), 4.35 (dd, $J=11.4,2.7 \mathrm{~Hz}, 1 \mathrm{H}), 3.86(\mathrm{~d}, J=11.2 \mathrm{~Hz}, 1 \mathrm{H}), 2.31$ (d, $J=8.4 \mathrm{~Hz}, 1 \mathrm{H})$, $1.57(\mathrm{~s}, 3 \mathrm{H}), 1.54(\mathrm{~s}, 3 \mathrm{H}) .{ }^{13} \mathrm{C} \mathrm{NMR}\left(101 \mathrm{MHz}, \mathrm{CDCl}_{3}\right) \delta 140.9,135.6,128.3,127.3$, 126.1, 117.4, 99.6, 73.3, 66.1, 45.0, 30.0, 19.4. The ${ }^{1} \mathrm{H}$ NMR and ${ }^{13} \mathrm{C}$ NMR spectroscopic data of compound $\mathbf{S 1}$ match the reported literature data. ${ }^{3}$

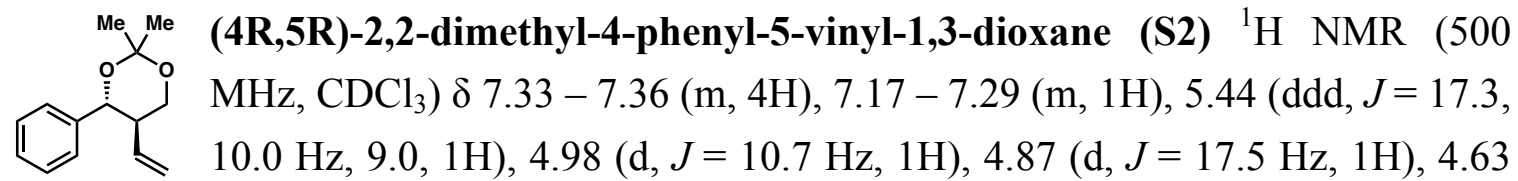
(d, $J=10.3 \mathrm{~Hz}, 1 \mathrm{H}), 3.63-4.13(\mathrm{~m}, 2 \mathrm{H}), 2.38-2.77(\mathrm{~m}, 1 \mathrm{H}), 1.58(\mathrm{~s}, 3 \mathrm{H}), 1.50(\mathrm{~s}, 3 \mathrm{H})$. ${ }^{13} \mathrm{C}$ NMR $\left(126 \mathrm{MHz}, \mathrm{CDCl}_{3}\right) \delta 140.4,134.3,128.6,128.4,127.8,118.7,99.1,76.8,64.4$, 46.4, 30.2, 19.4. The ${ }^{1} \mathrm{H}$ NMR and ${ }^{13} \mathrm{C}$ NMR spectroscopic data of compound $\mathbf{S 1}$ match the reported literature data. ${ }^{3}$

\section{Assignment of the absolute configuration using Mosher ester analysis:}

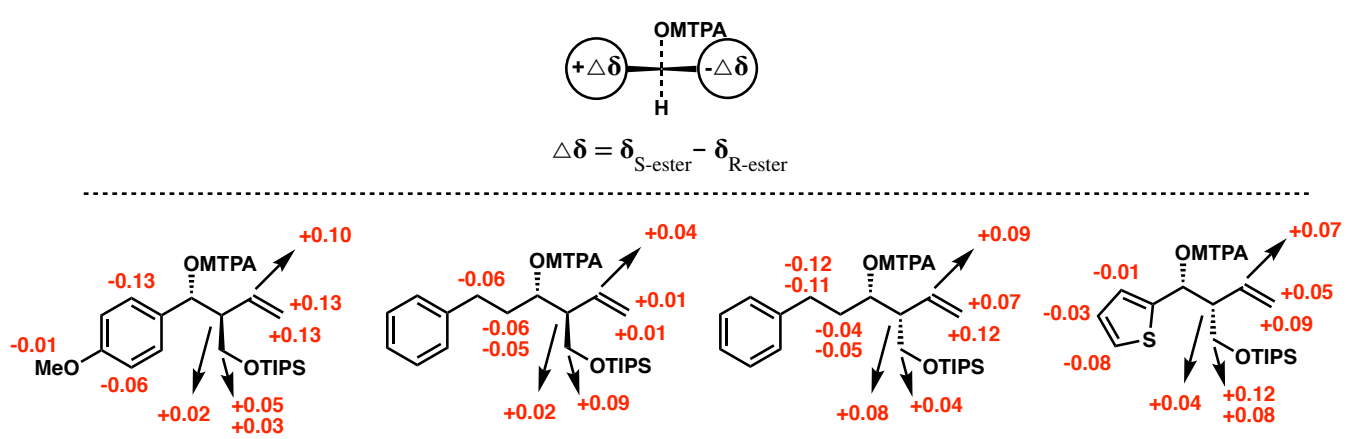




\section{Stoichiometric reaction of preformed IPrCuCl complex with diene 7}

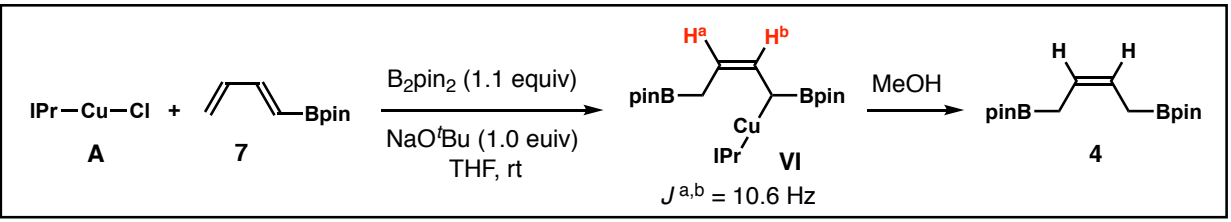

In an Ar-filled glove box, preformed $\mathrm{IPrCuCl}$ complex $\mathbf{A}$ (1.0 equiv) was treated with $\mathrm{NaO}^{t} \mathrm{Bu}$ (1.0 equiv) and $\mathrm{B}_{2} \mathrm{Pin}_{2}$ (1.1 equiv) in THF at ambient temperature for 5 minutes. Then diene 7 (1.0 equiv) was added, and the solution was stirred for 10 minutes. The formation of $Z$-allylcopper VI was confirmed by ${ }^{1} \mathrm{H}$ NMR analysis (in $d^{8}$-toluene). The signals of two olefinic protons were observed $\left(\delta \mathrm{H}^{\mathrm{a}}: 4.60 \mathrm{ppm}, \mathrm{H}^{\mathrm{b}}: 6.13 \mathrm{ppm}, J^{\mathrm{a}, \mathrm{b}}=10.6\right.$ $\mathrm{Hz}$ ). The coupling constant is consistent with $Z$-alkene geometry. After the addition of $\mathrm{MeOH}$ (1.0 equiv), the olefinic signals of $Z$-allylcopper VI disappeared, and the signal of new olefinic proton appeared $(\delta 5.73 \mathrm{ppm})$, which indicates that protonation of VI with $\mathrm{MeOH}$ generated allylboronate 4. These data support our analysis of the formation of $Z$-allylcopper species VI via 1,4-borocupration of diene 7, and a subsequent direct $\mathrm{S}_{\mathrm{E}} 2$ protonation of $\mathbf{V I}$ to generate $Z$-allylboronate 4 .

\section{References:}

1. Gao, S.; Chen, M. Chem. Commun. 2019, 11199.

2. Su, B.-N.; Takaishi, Y.; Kusumi, T. Tetrahedron 1999, 55, 14571.

3. Kister, J.; Ess, D. H.; Roush, W. R. Org. Lett. 2013, 15, 5436. 

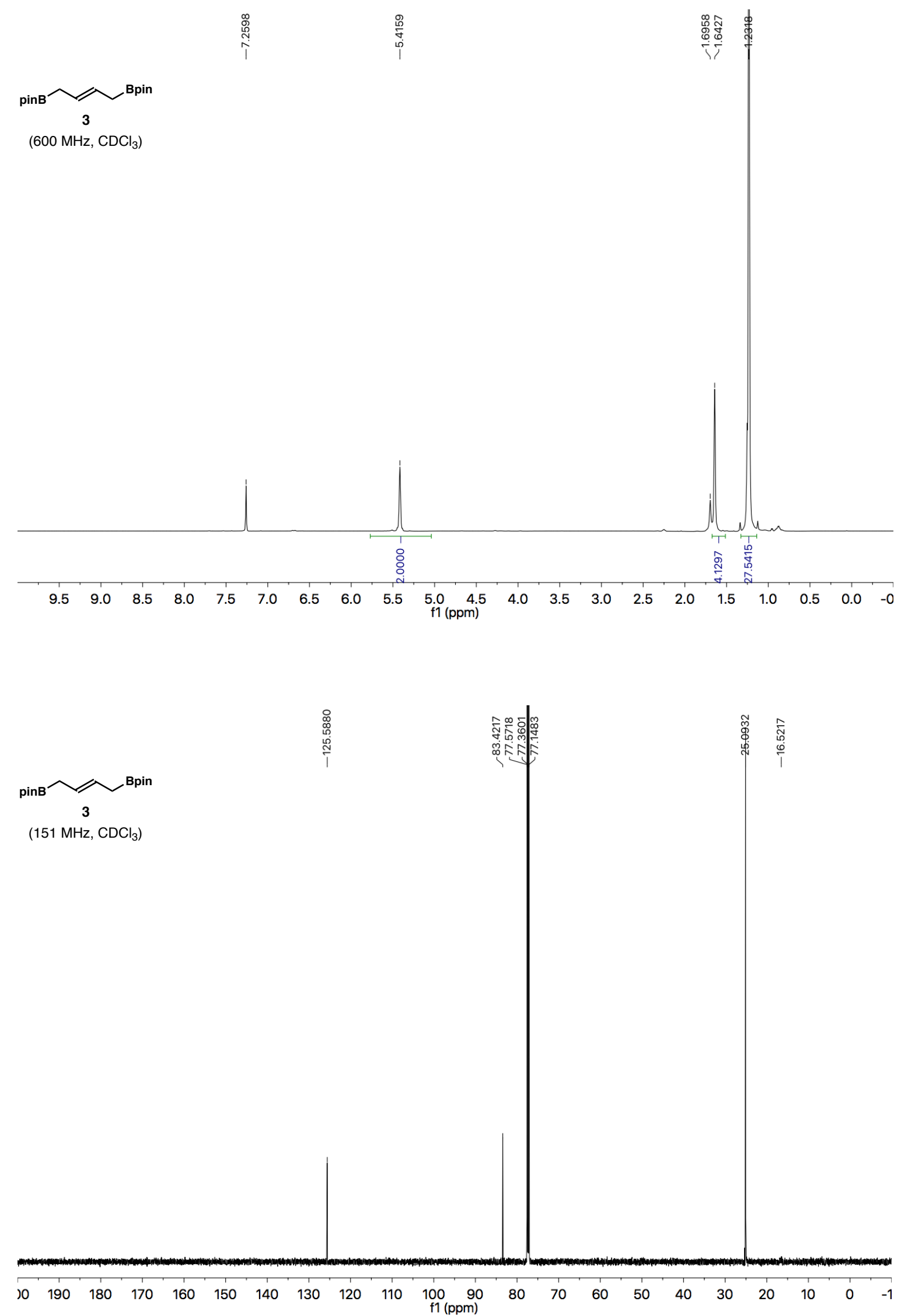

SI-28 
pinB $-\overbrace{4}{ }_{\text {Bpin }}$

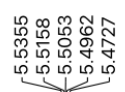

(500 MHz, $\mathrm{CDCl}_{3}$ )
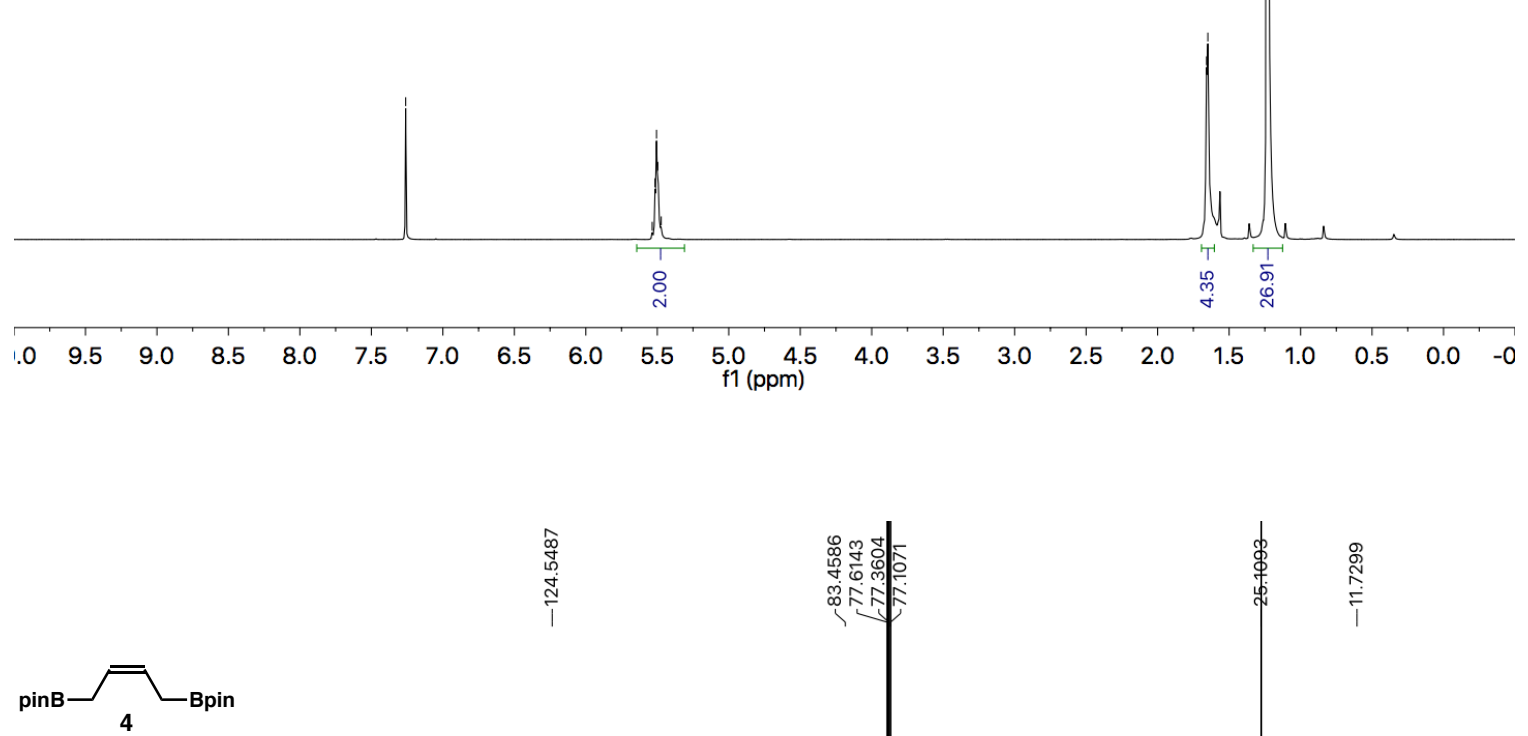

$\left(126 \mathrm{MHz}, \mathrm{CDCl}_{3}\right.$ )

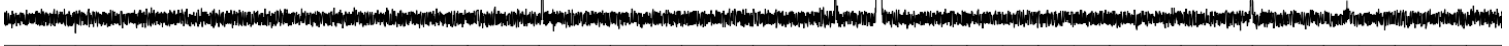

$\begin{array}{llllllllllllllllllllll}10 & 190 & 180 & 170 & 160 & 150 & 140 & 130 & 120 & 110 & \begin{array}{c}100 \\ \mathrm{f} 1(\mathrm{ppm})\end{array} & 80 & 70 & 60 & 50 & 40 & 30 & 20 & 10 & 0 & -1\end{array}$ 

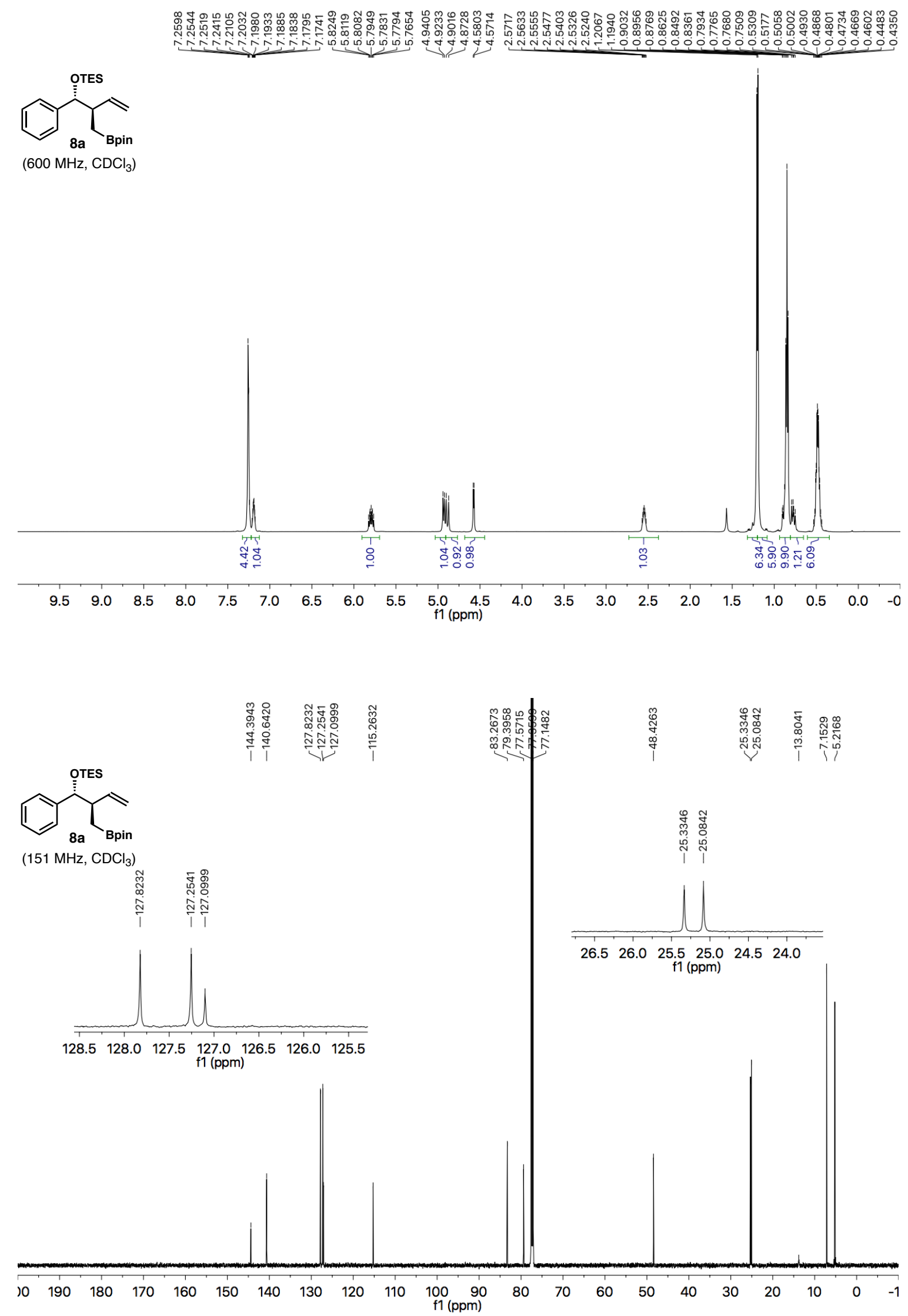

SI-30 

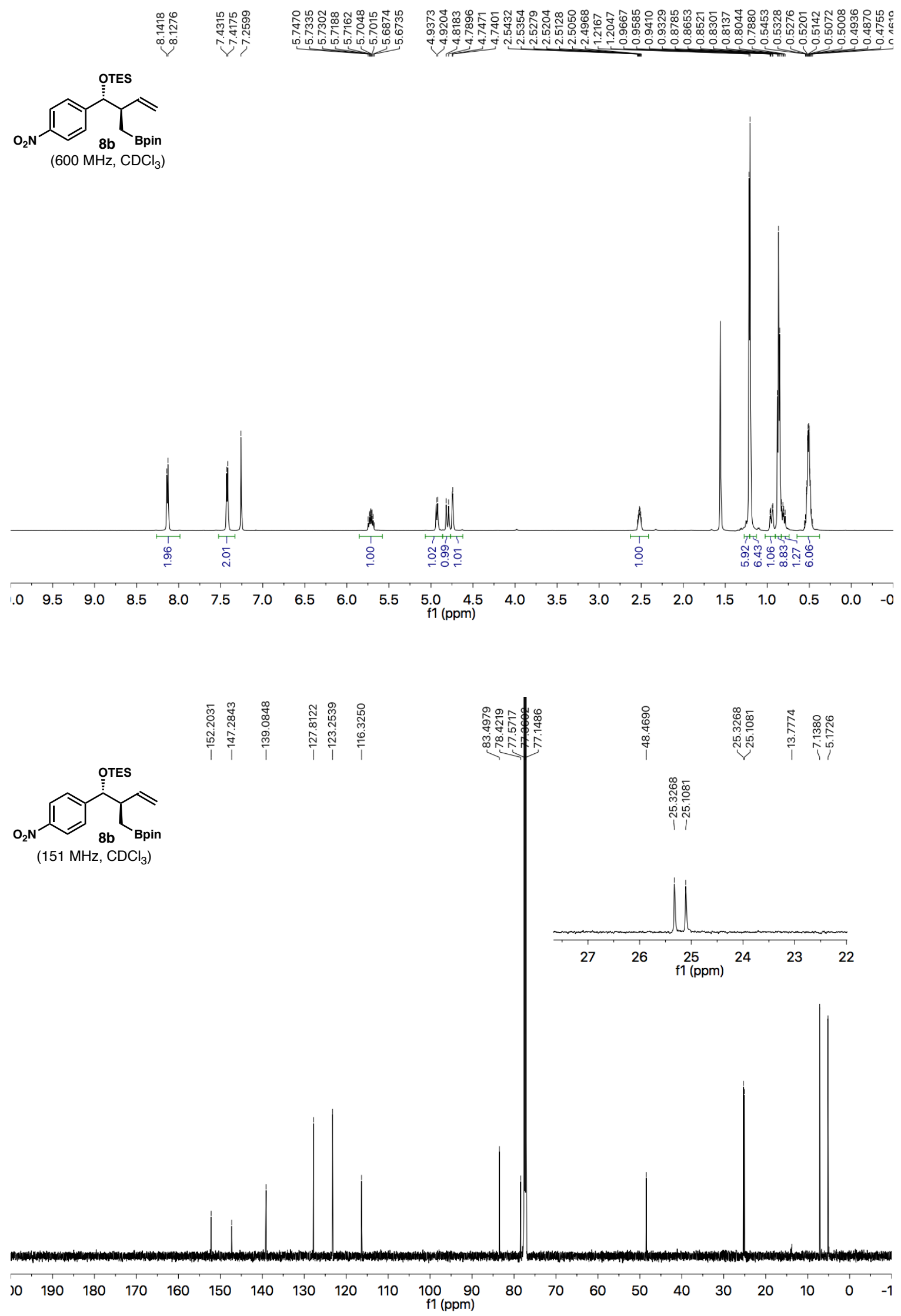

SI-31 

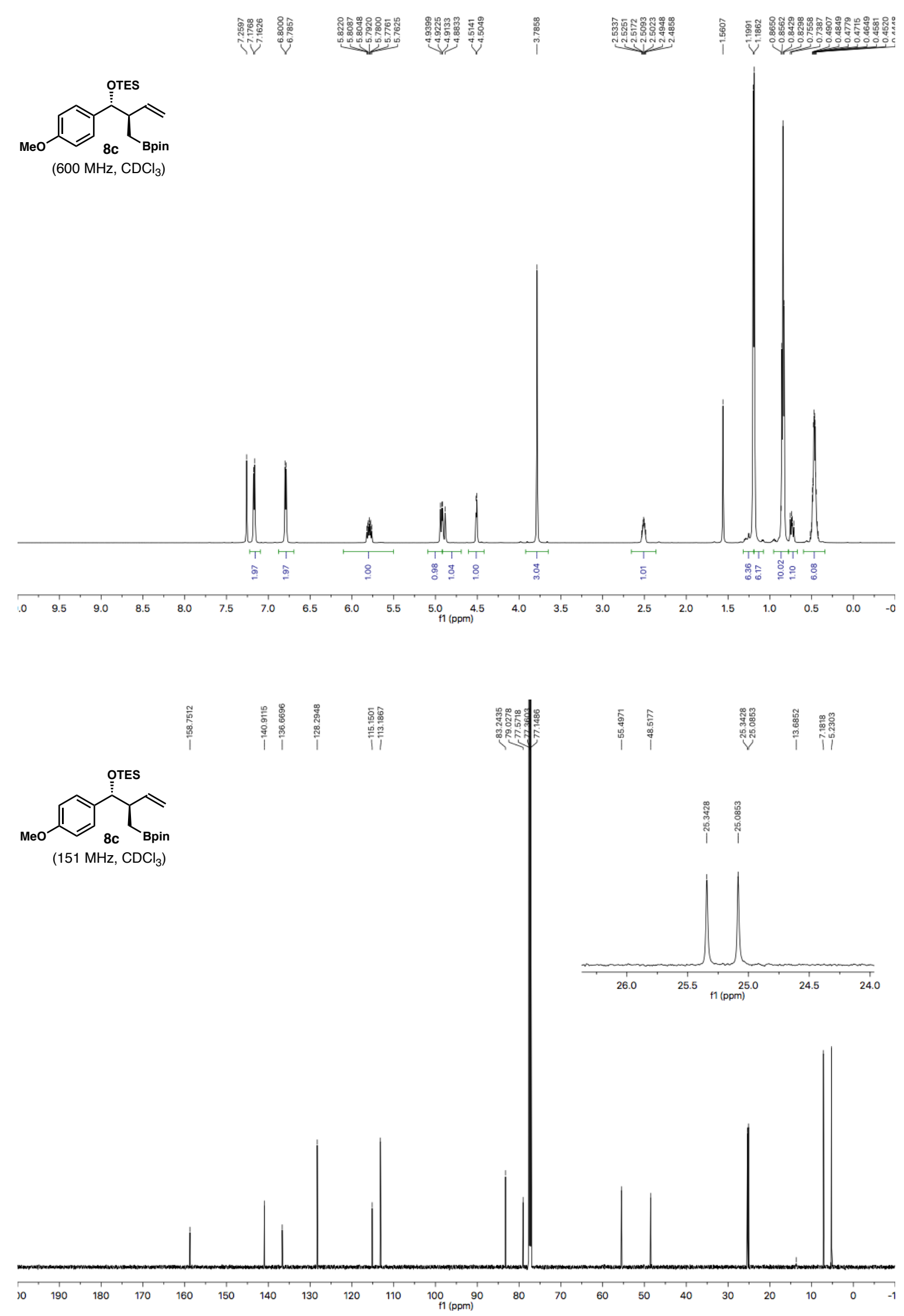

SI-32 


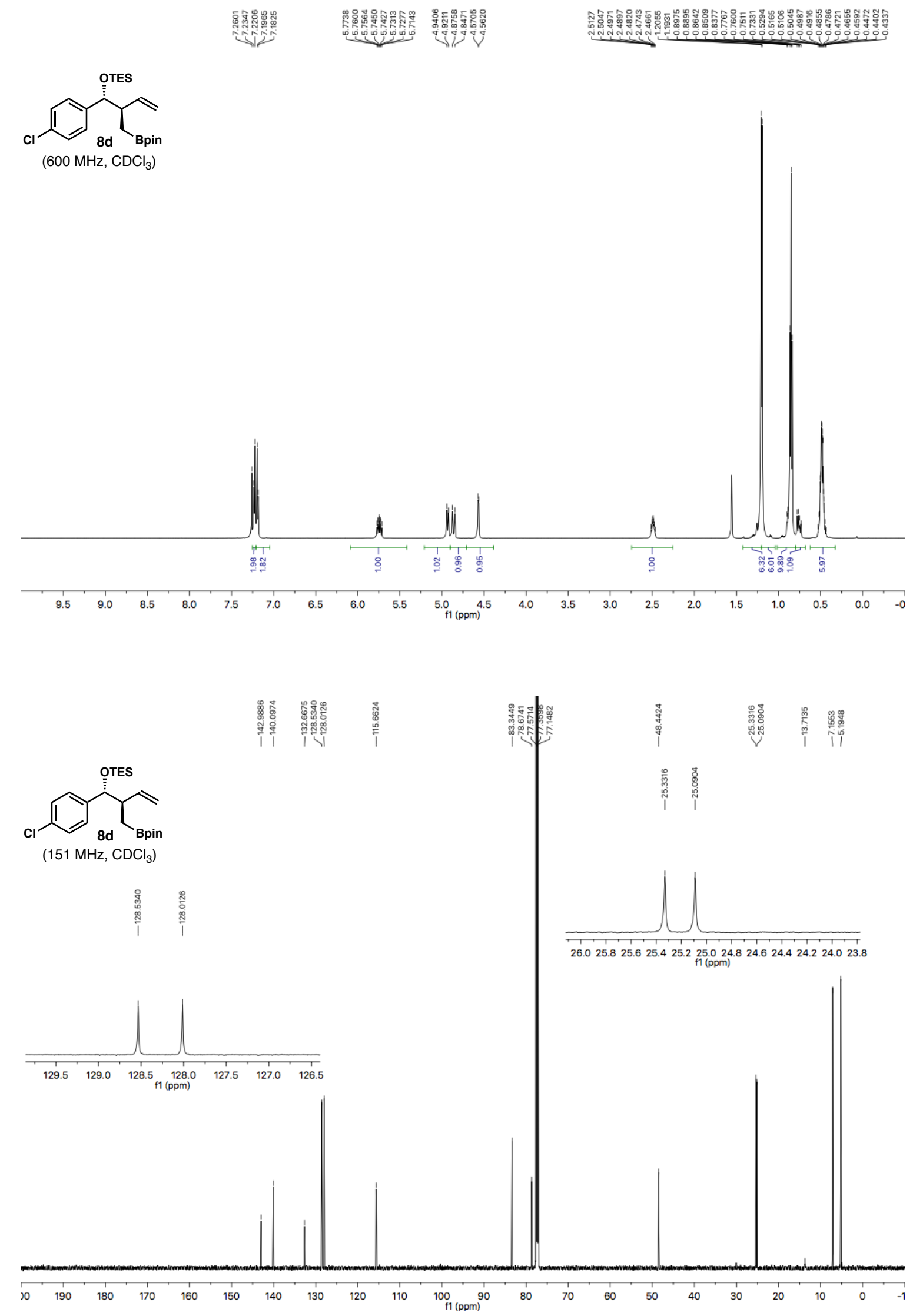

SI-33 

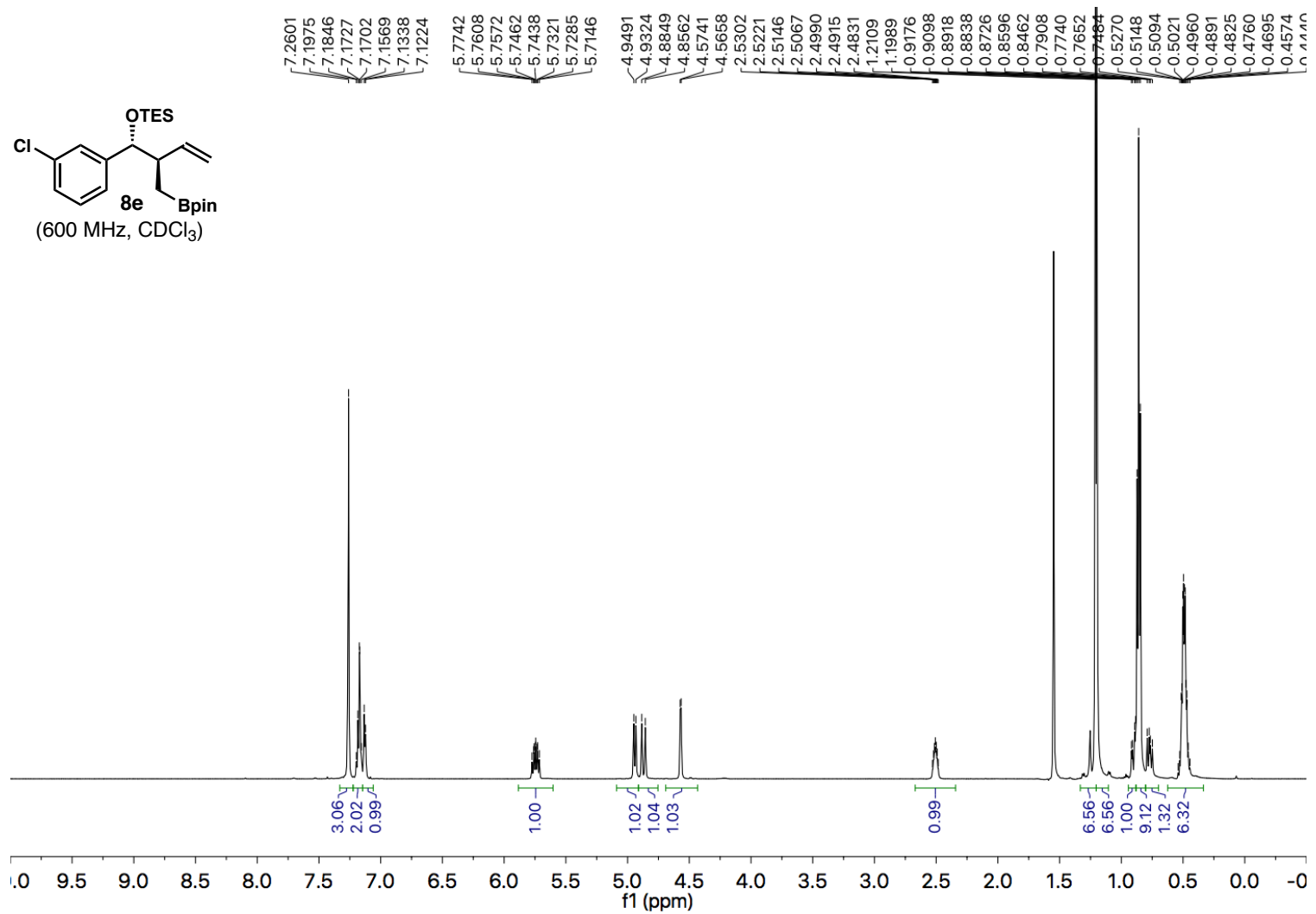

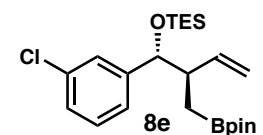

$\left(151 \mathrm{MHz}, \mathrm{CDCl}_{3}\right)$

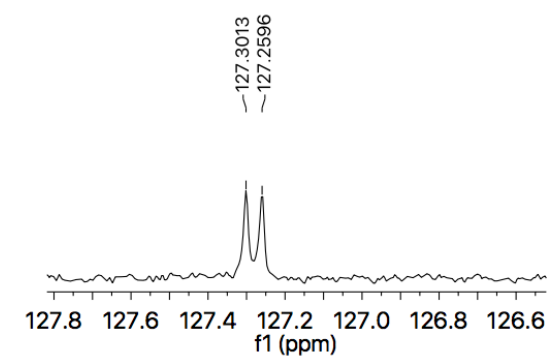

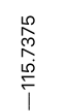

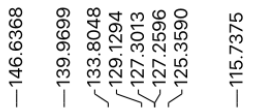

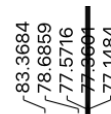

更

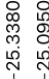

i i

$\begin{array}{llllllll}27.0 & 26.5 & 26.0 & 25.5 & 25.0 & 24.5 & 24.0 & 23.5\end{array}$ f1 (ppm)

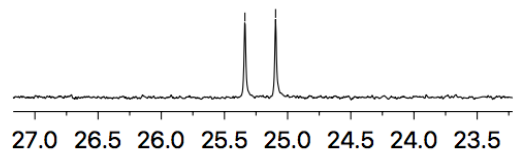

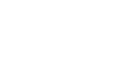

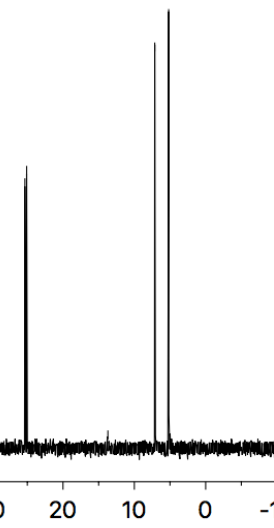



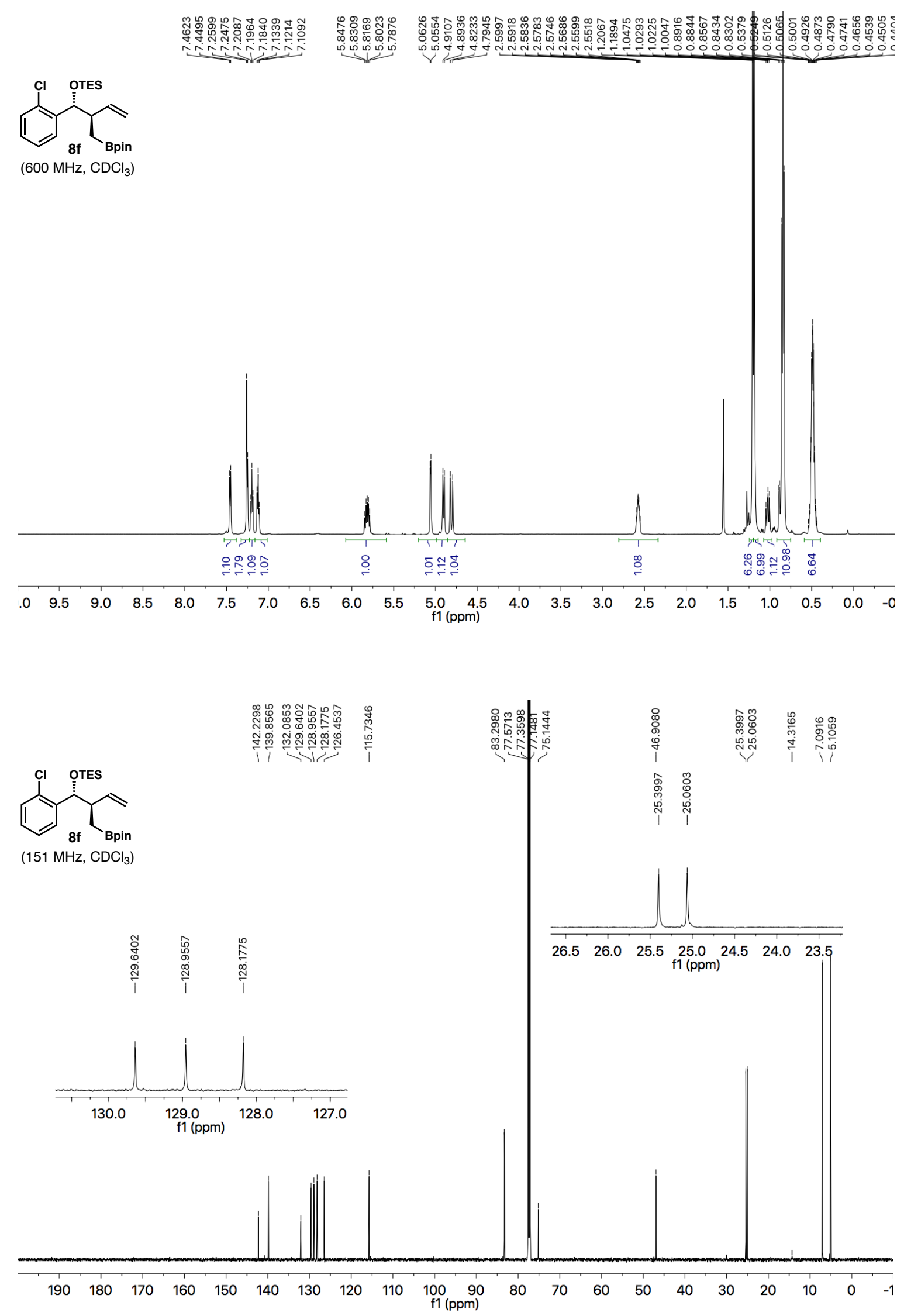

SI-35 

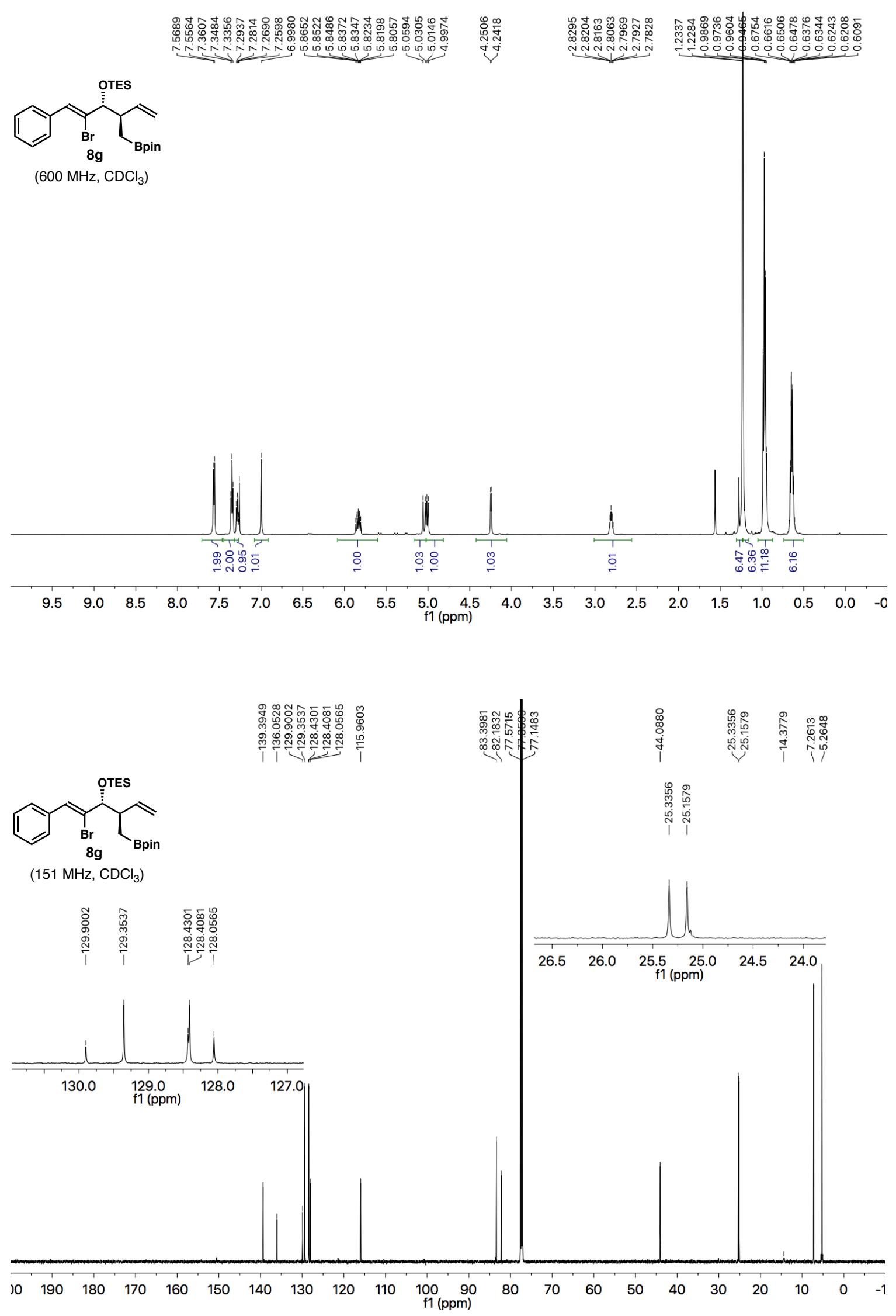

SI-36 

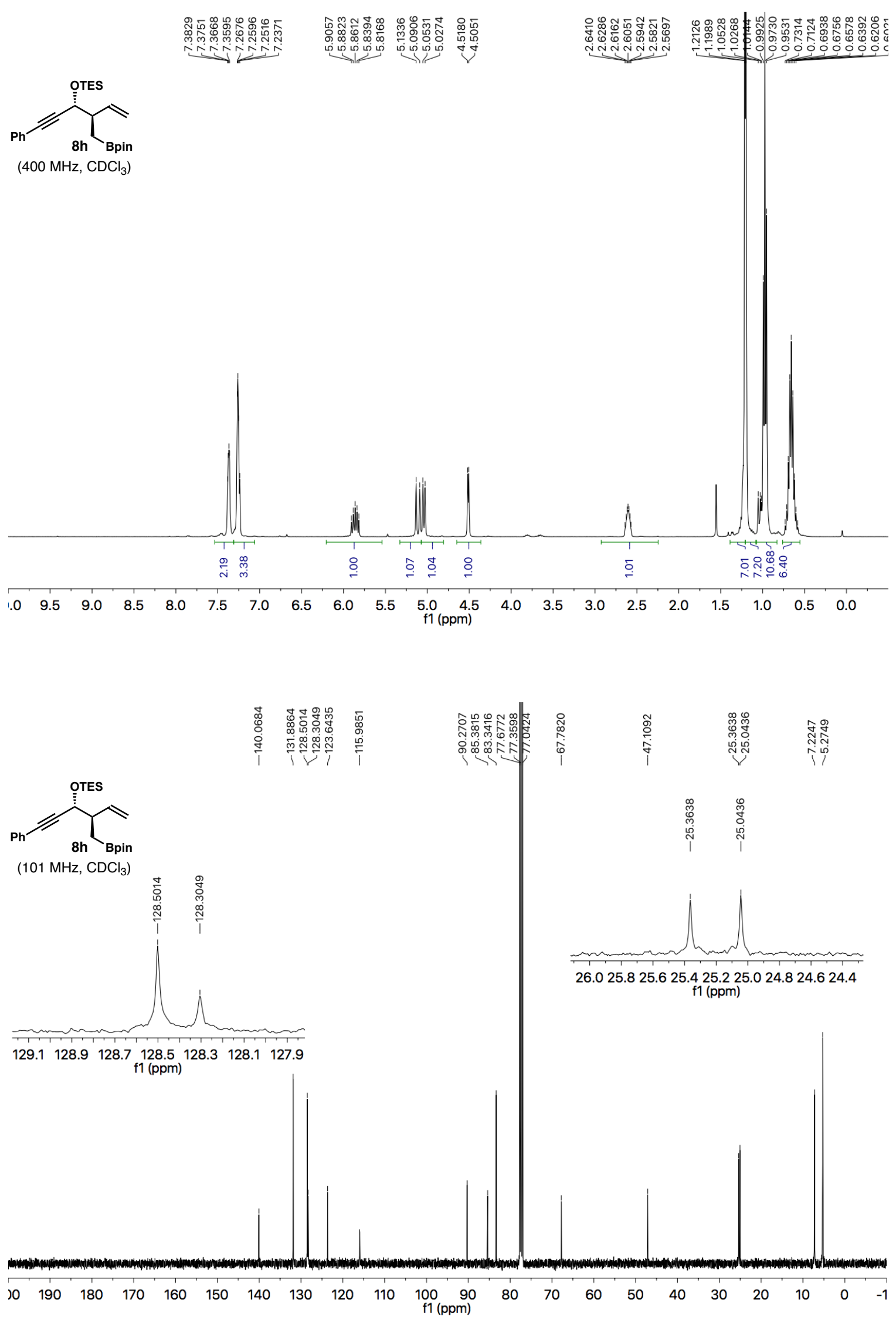

SI-37 

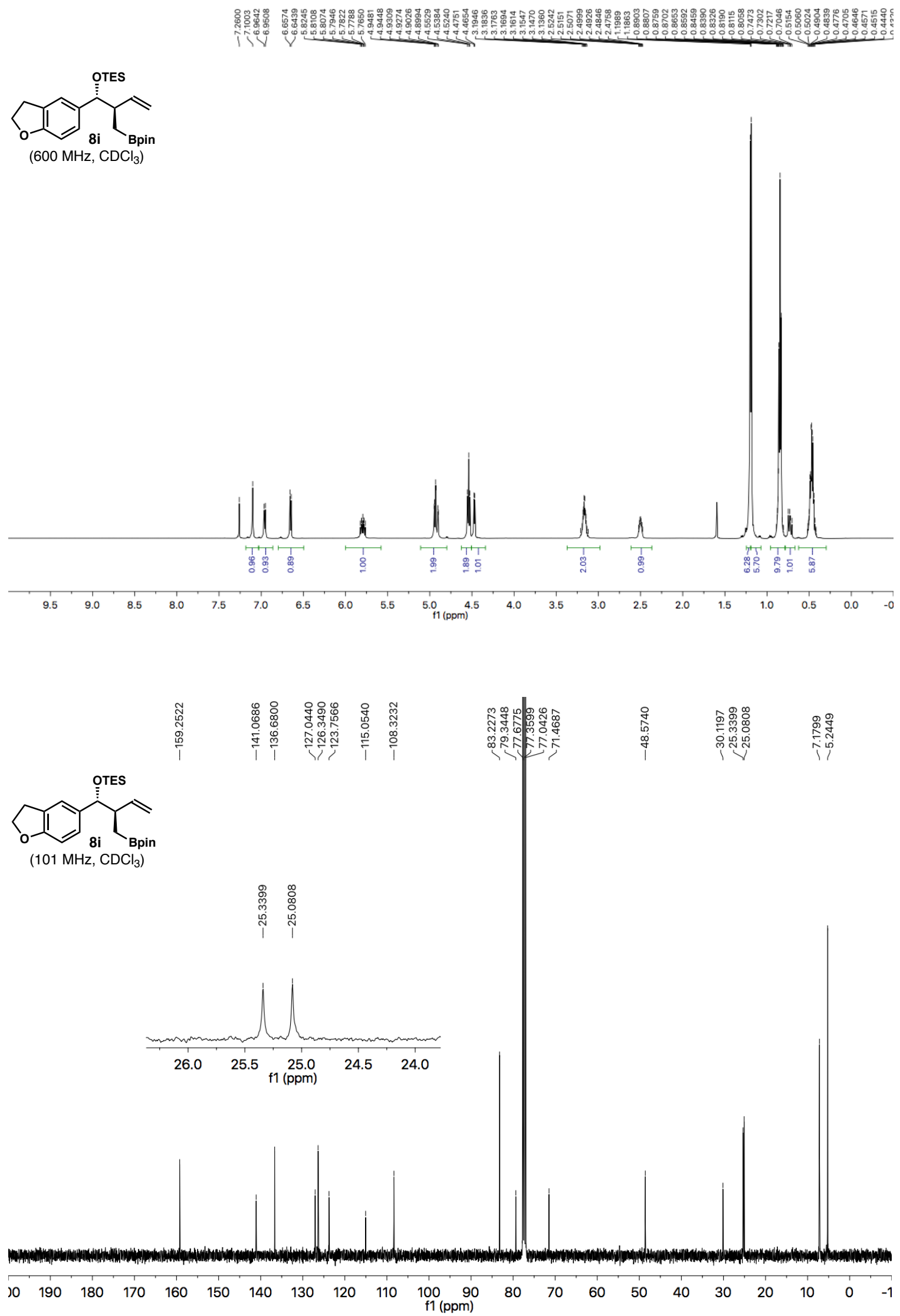

SI-38 

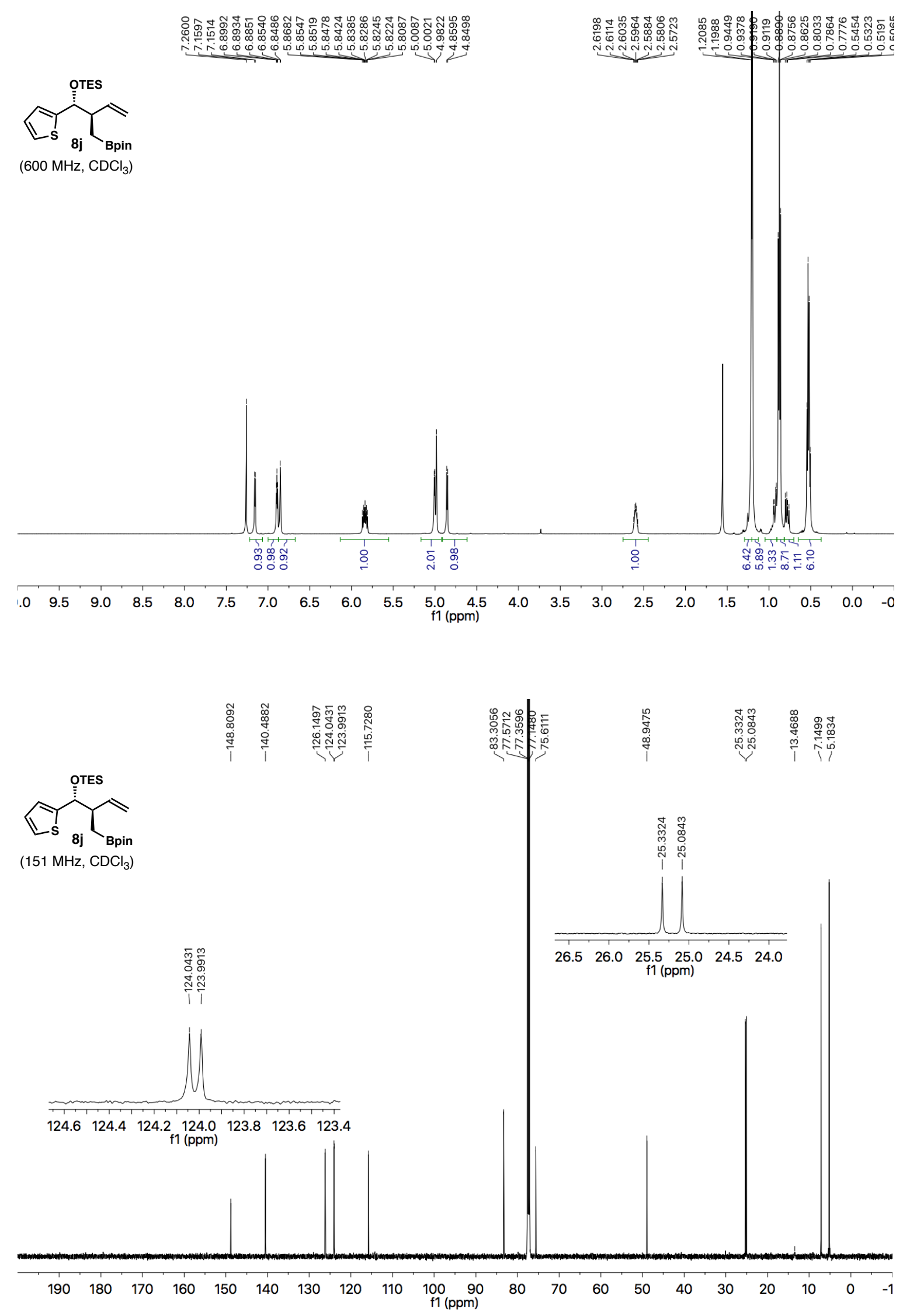

SI-39 

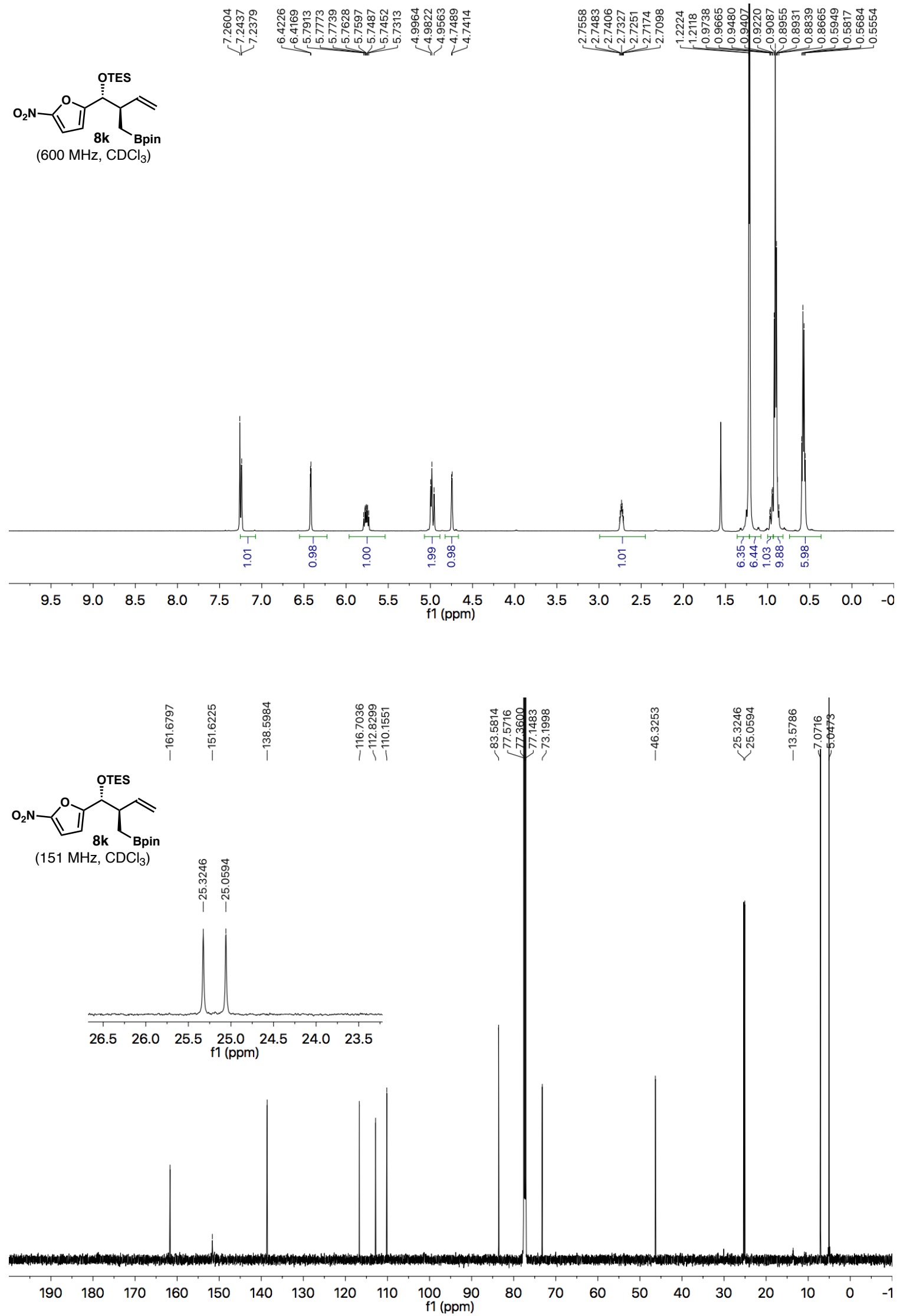

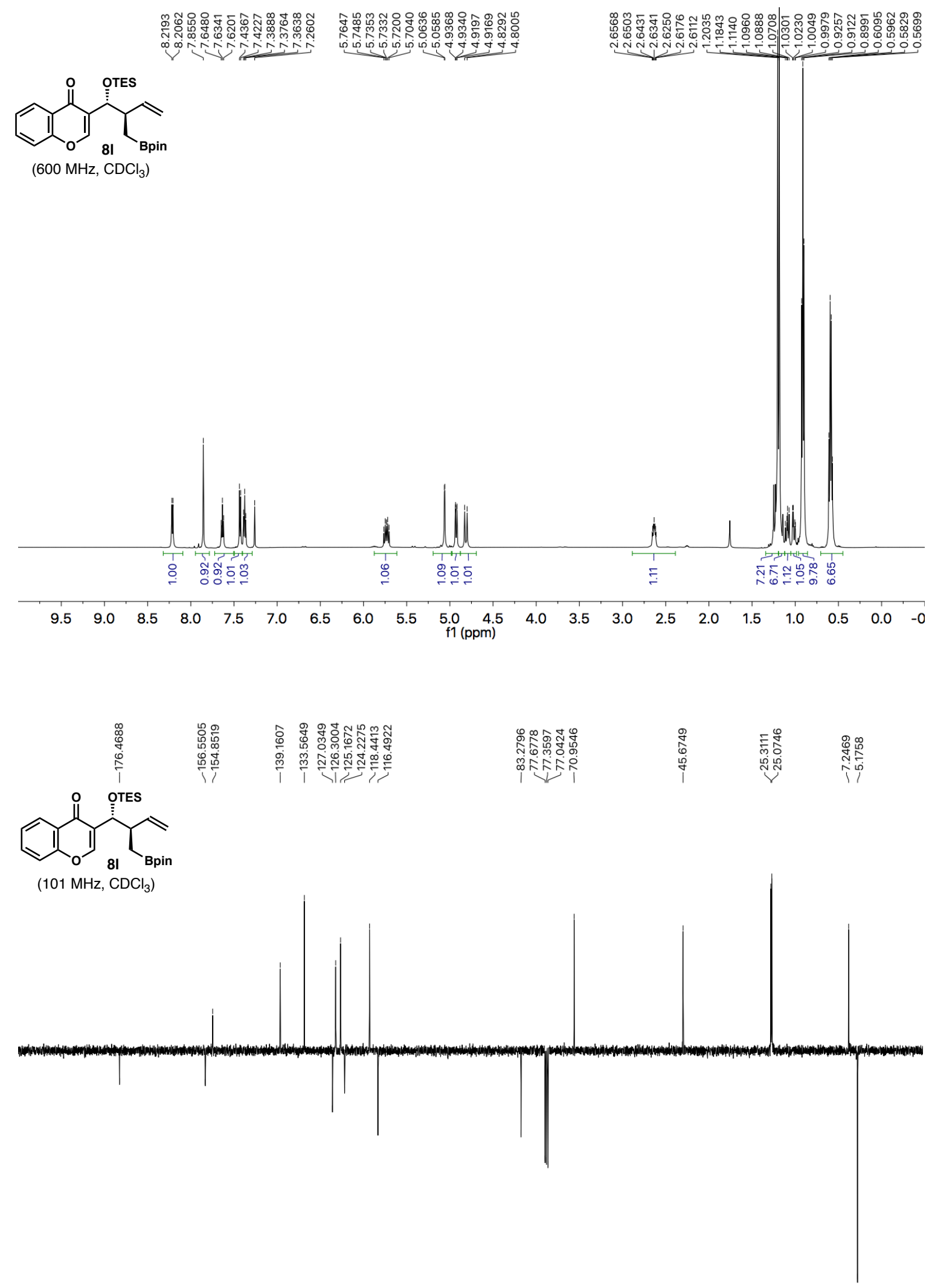

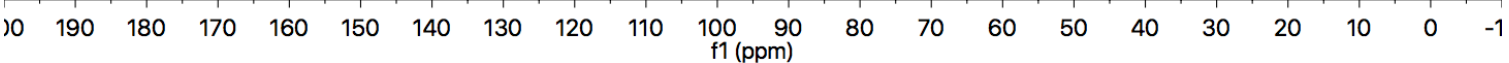



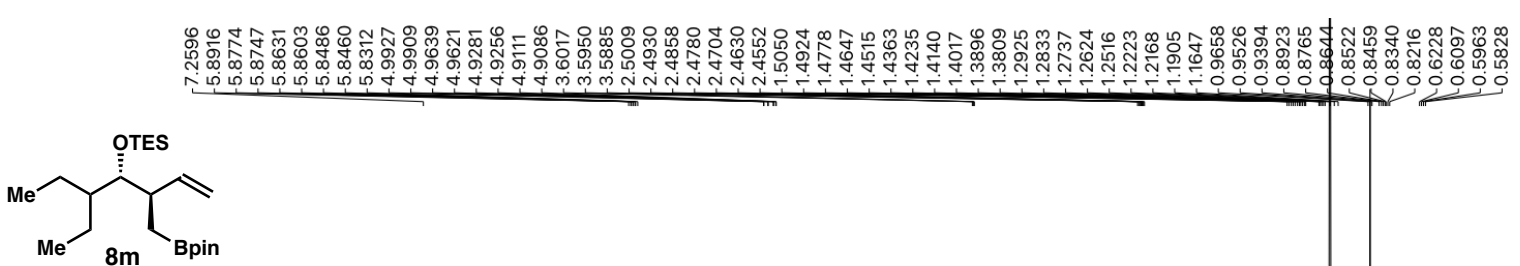

$\left(600 \mathrm{MHz}, \mathrm{CDCl}_{3}\right.$ )
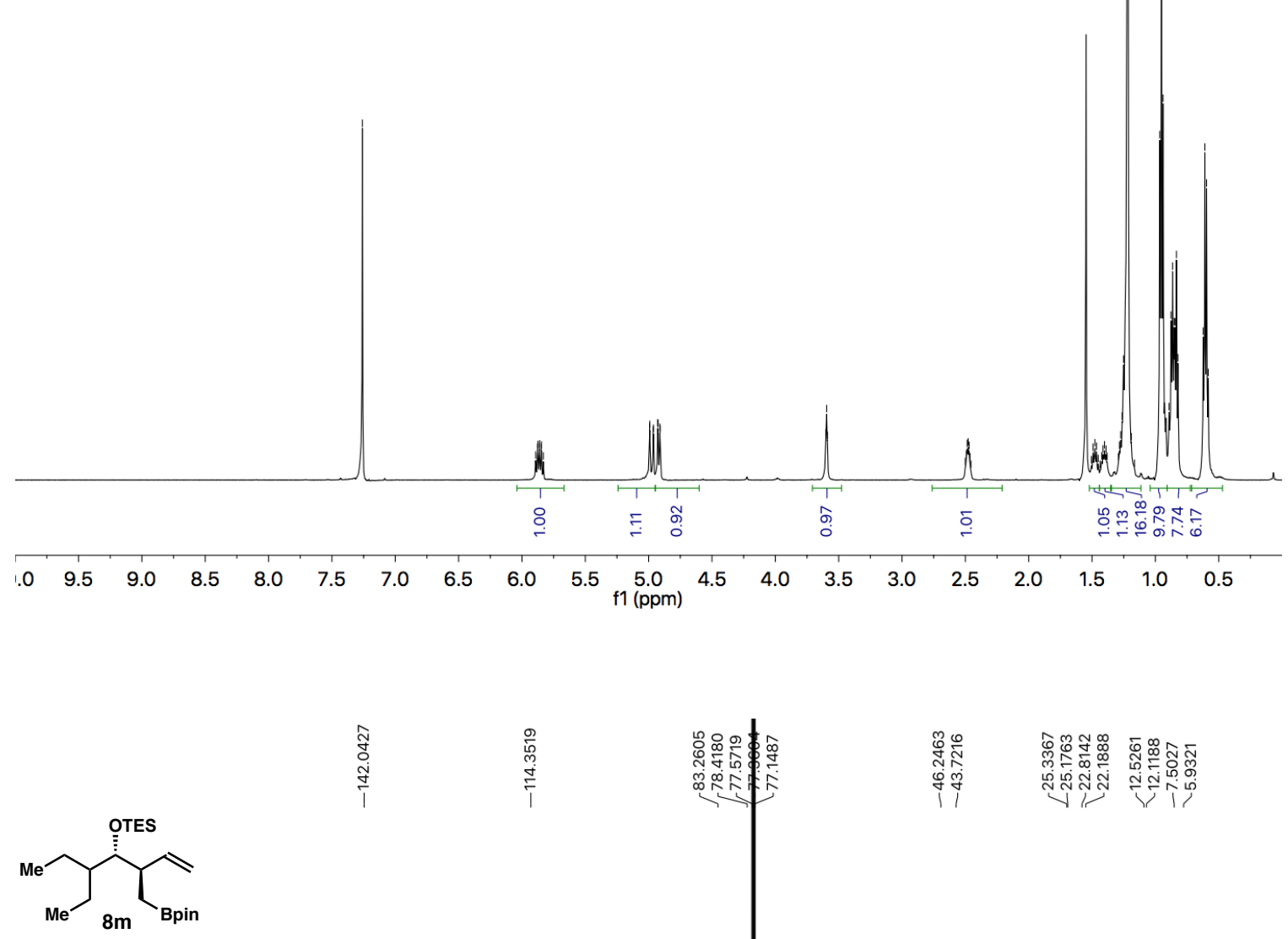

(151 MHz, $\mathrm{CDCl}_{3}$ )
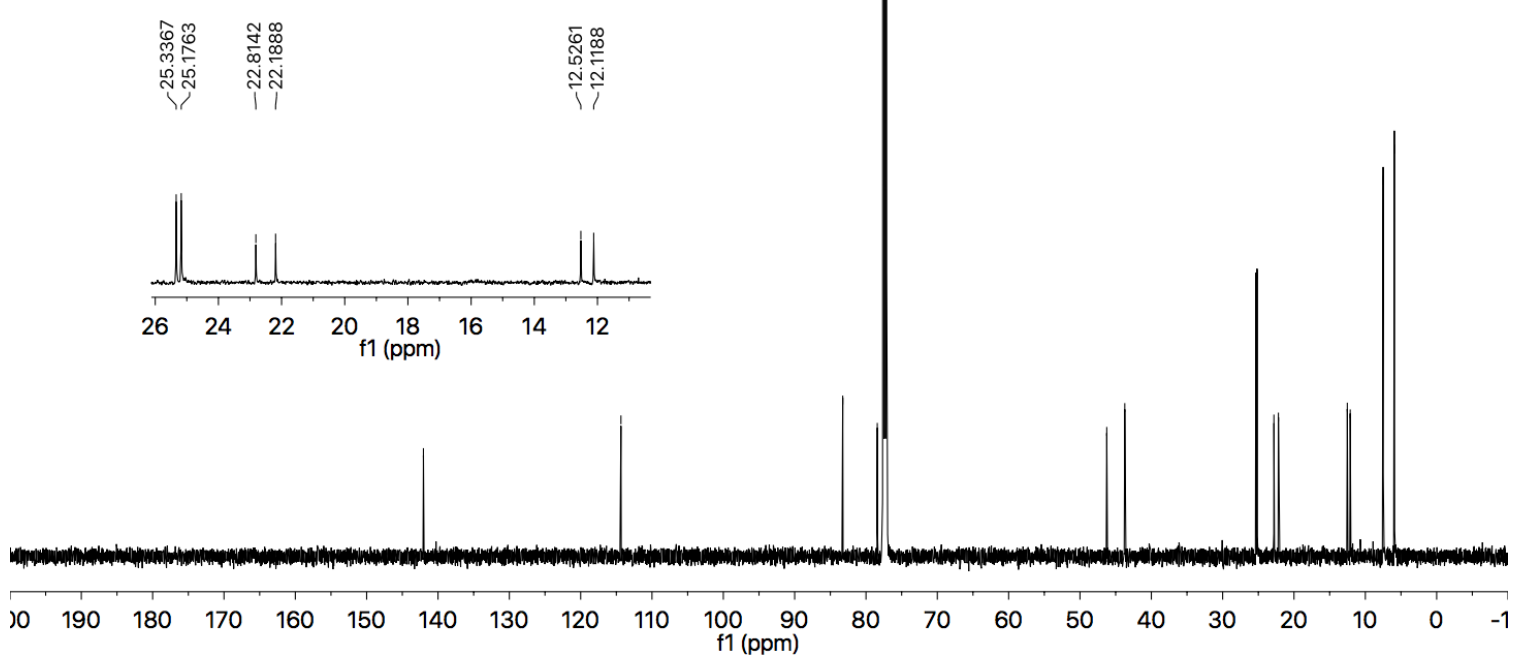

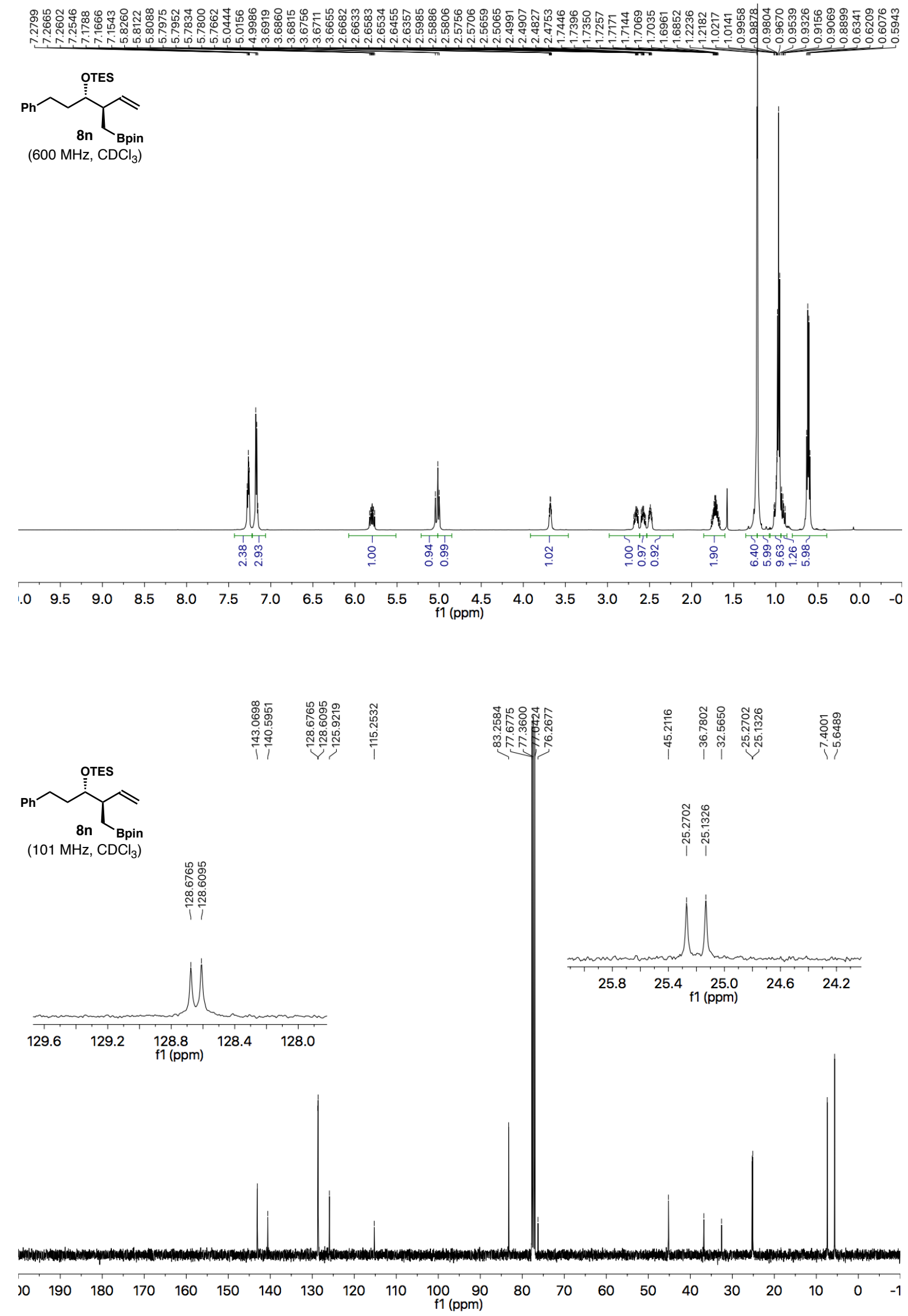

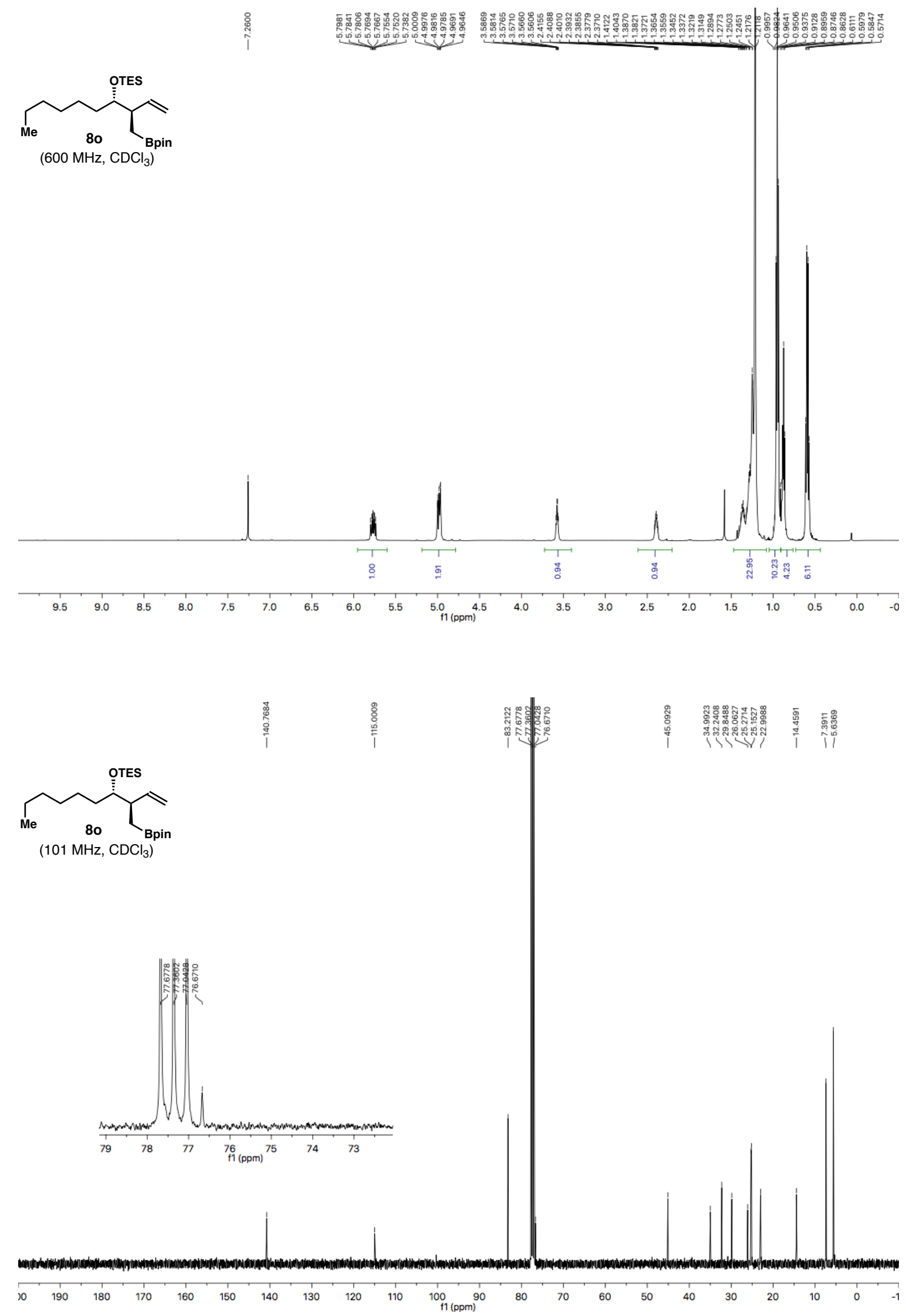

SI-44 


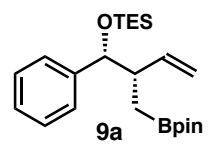

$\left(400 \mathrm{MHz}, \mathrm{CDCl}_{3}\right.$ )
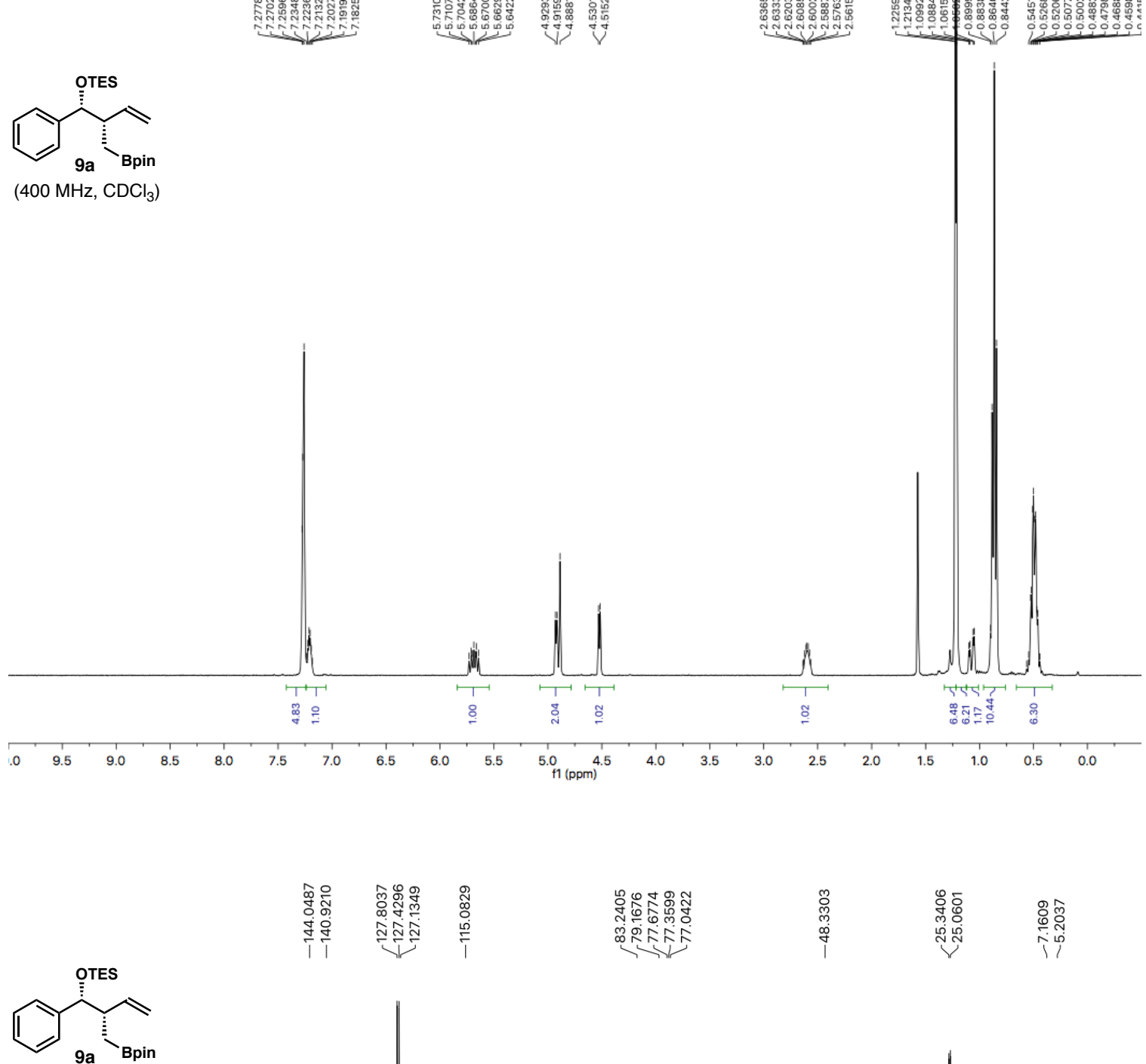

$\left(101 \mathrm{MHz}, \mathrm{CDCl}_{3}\right.$ )
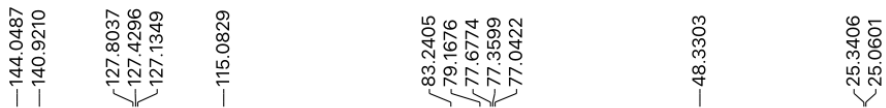

일
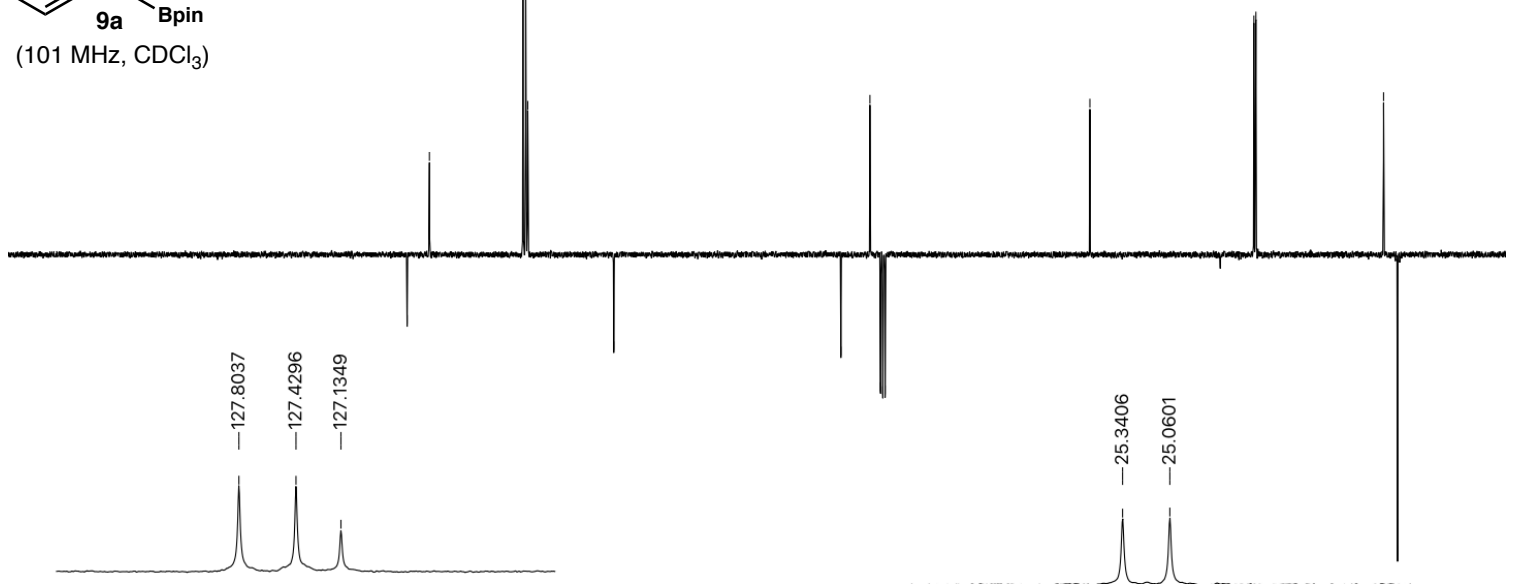

$\begin{array}{lllllll}129.0 & 128.5 & 128.0 & \begin{array}{l}127.5 \\ f 1(\mathrm{ppm})\end{array} & 127.0 & 126.5 & 126.0\end{array}$

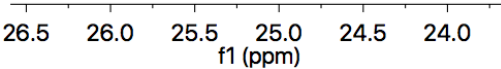

$\begin{array}{lllllllllll}10 & 190 & 180 & 170 & 160 & 150 & 140 & 130 & 120 & 110 & 100\end{array}$ 

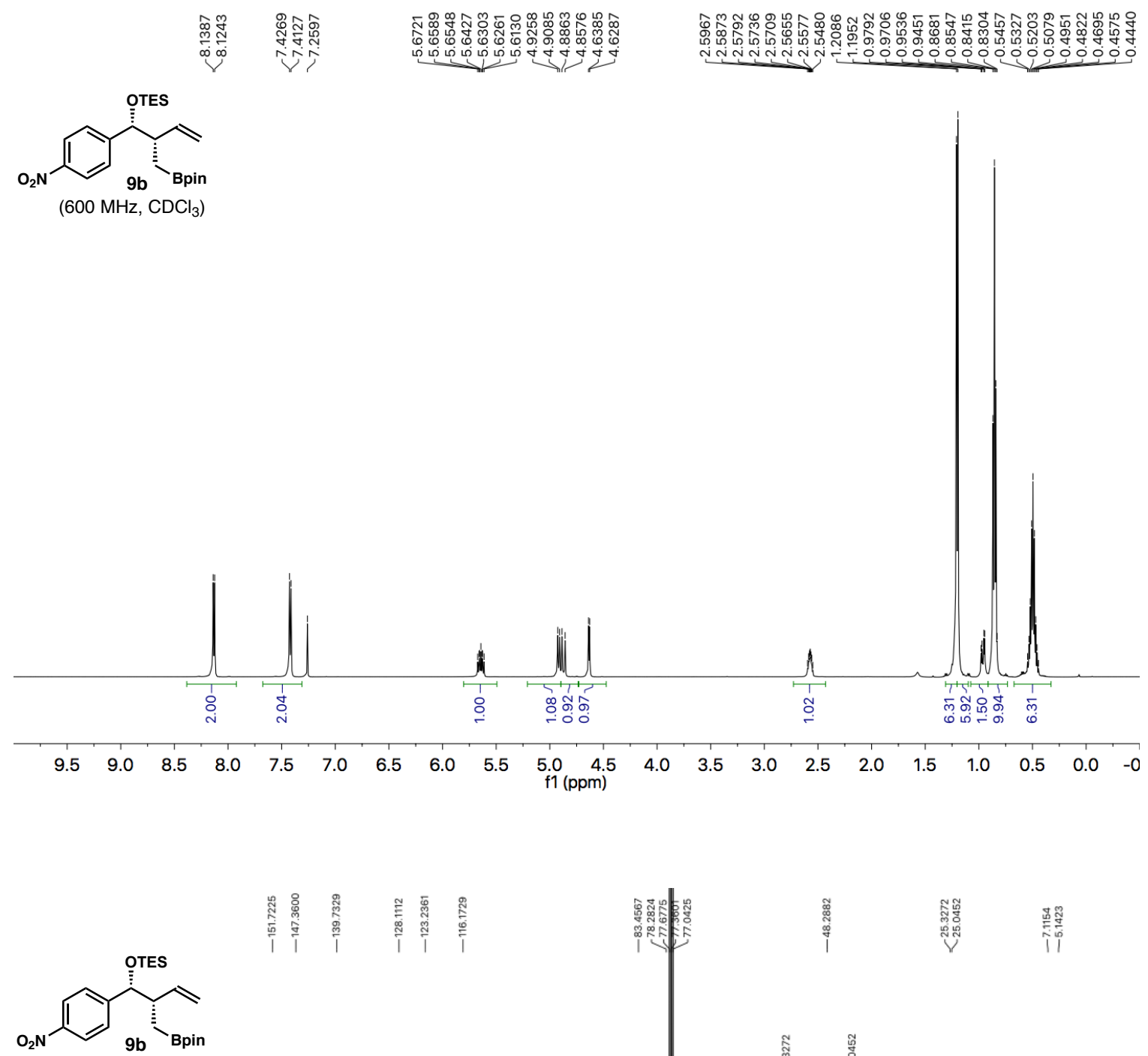

(101 MHz, $\mathrm{CDCl}_{3}$ )
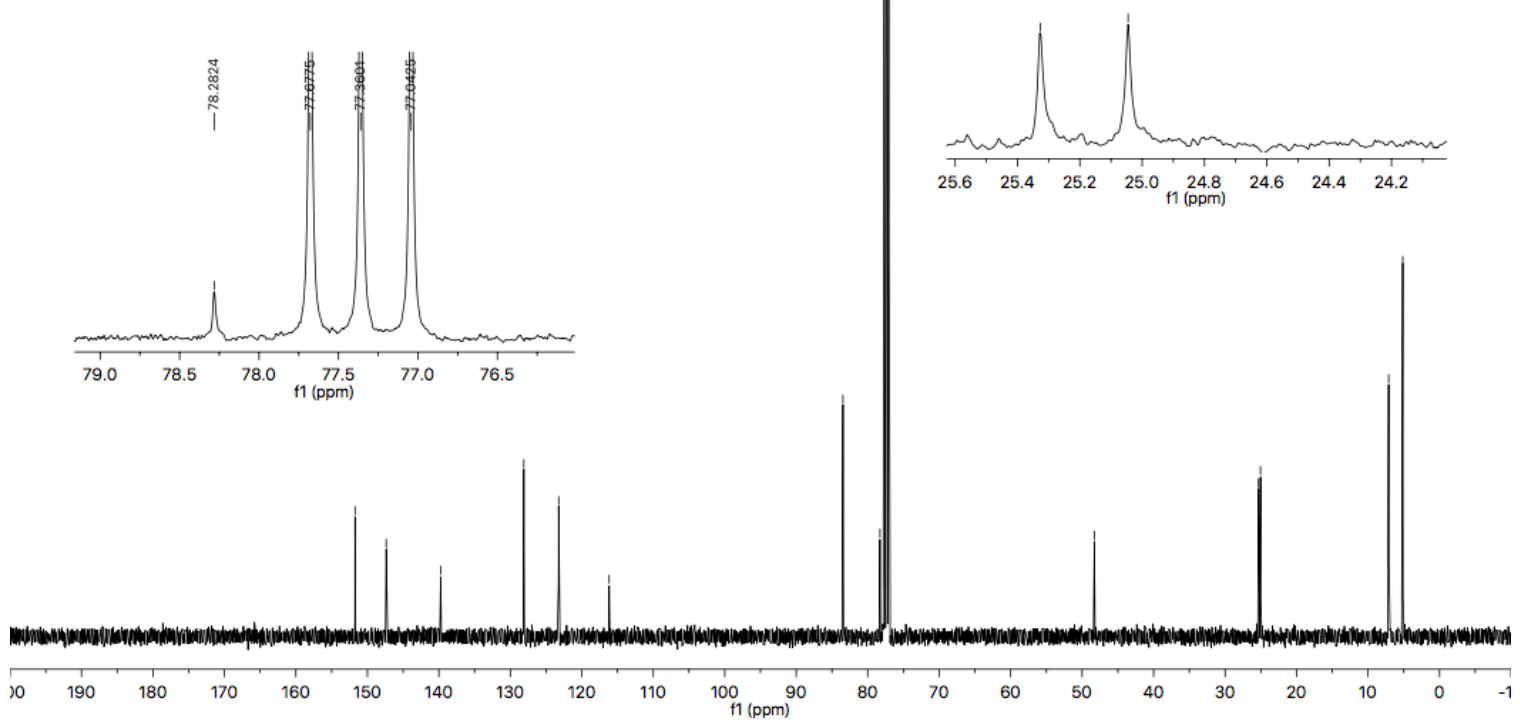

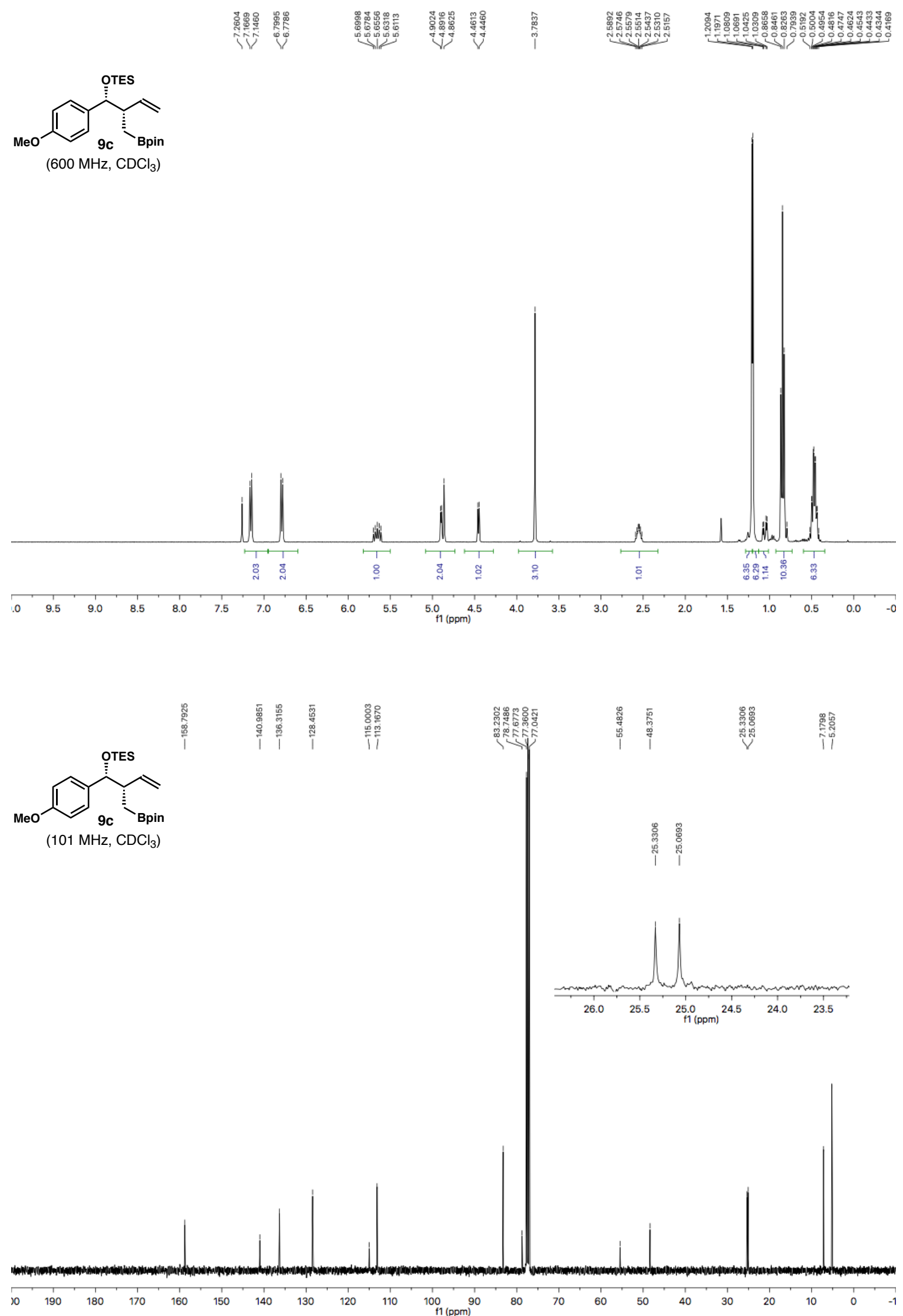

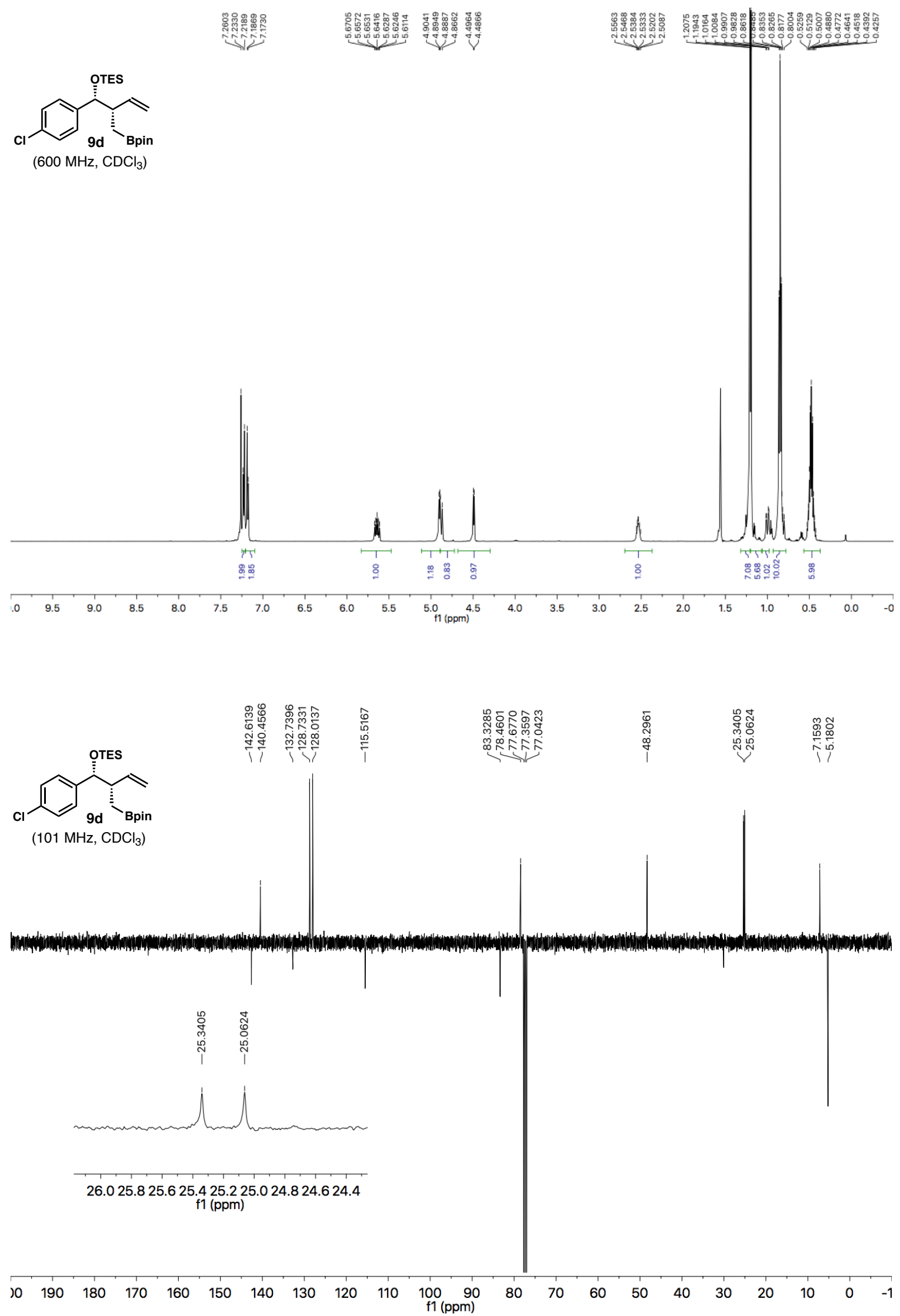


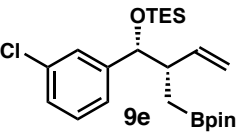

$\left(600 \mathrm{MHz}, \mathrm{CDCl}_{3}\right.$ )
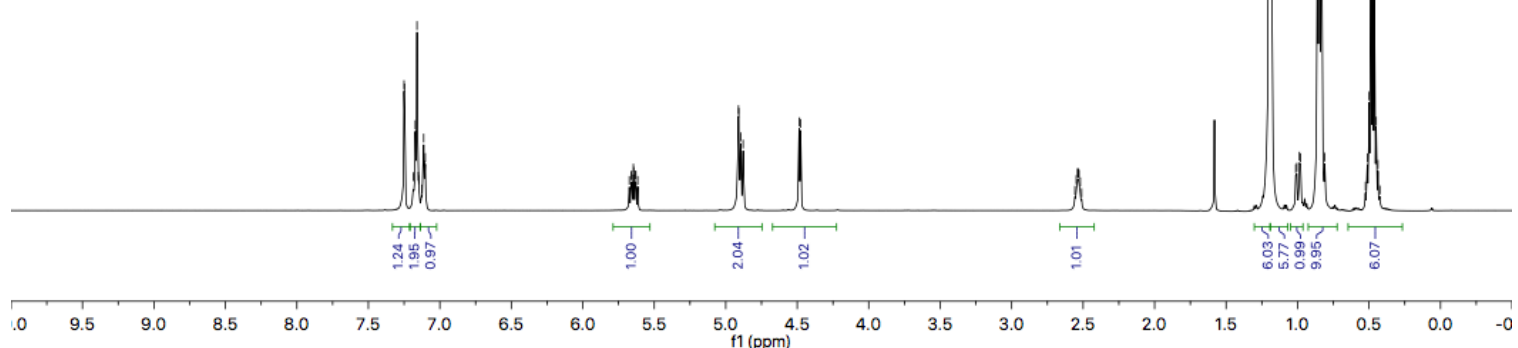

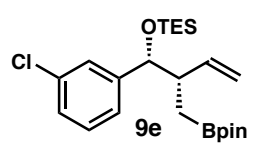

$\left(151 \mathrm{MHz} \mathrm{CDCl}_{3}\right.$ )
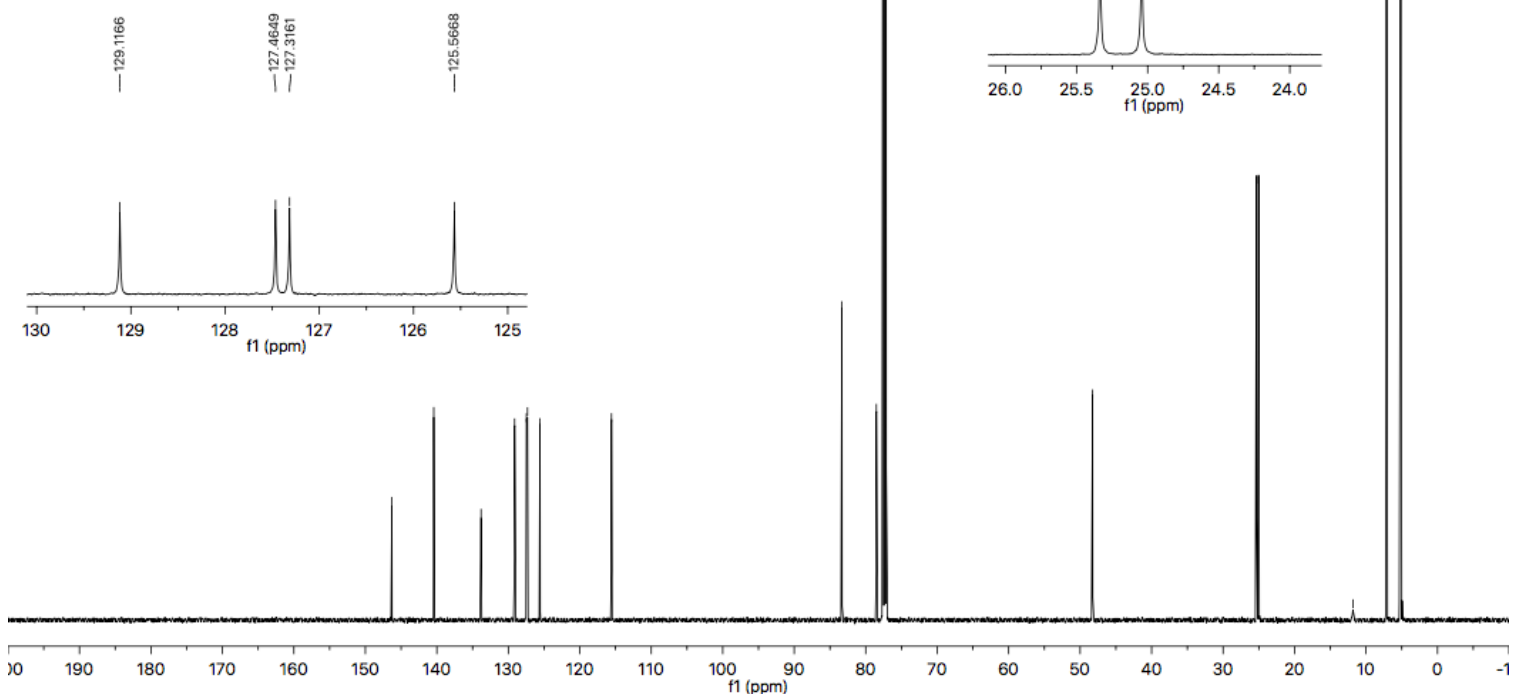


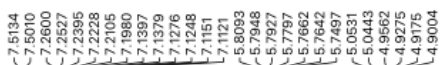

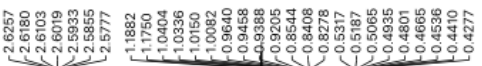
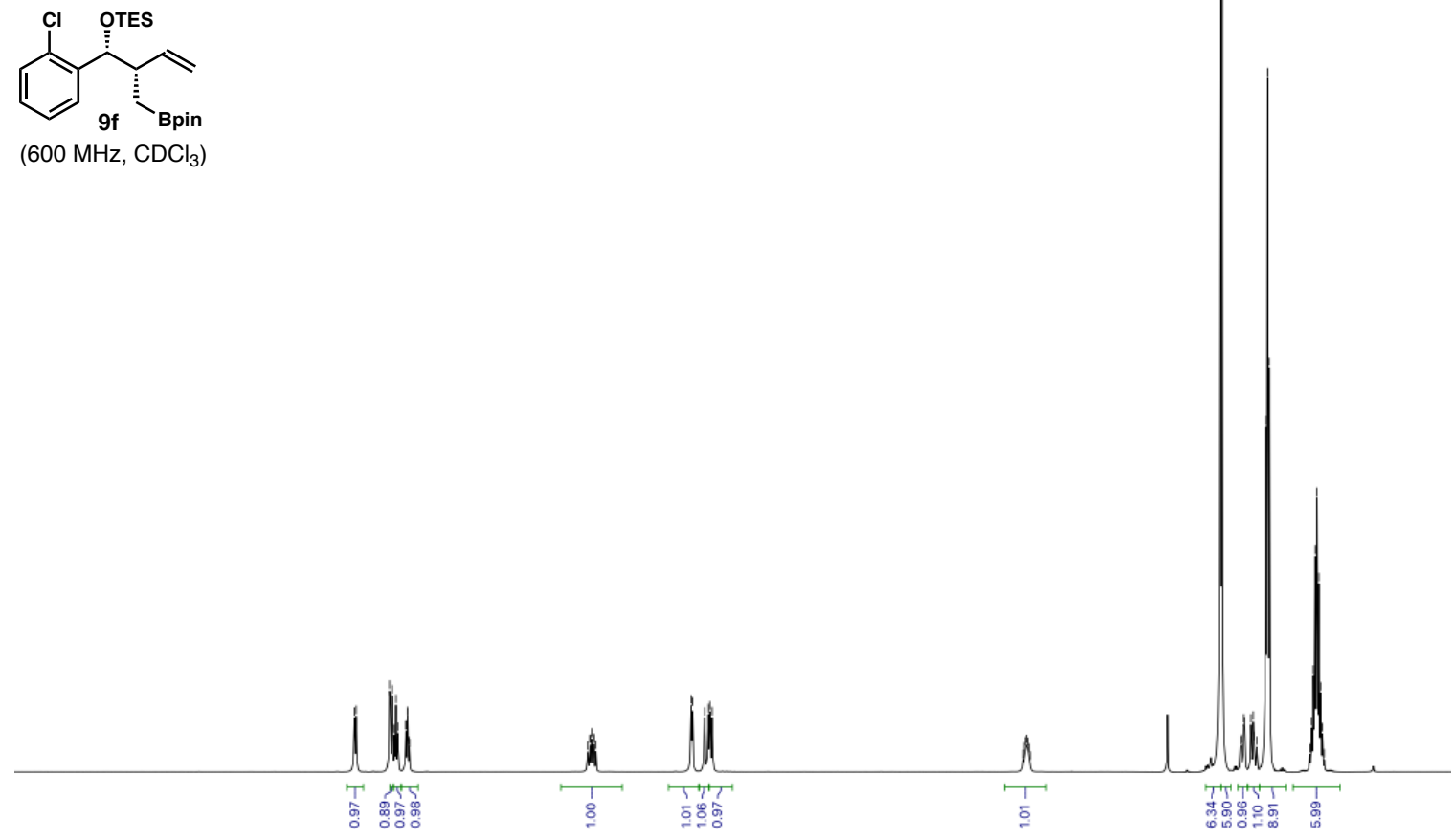

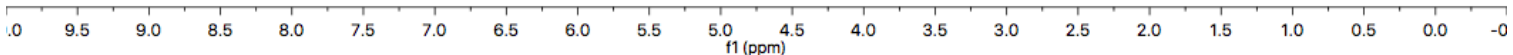

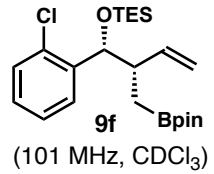

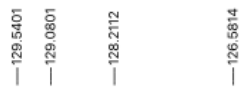

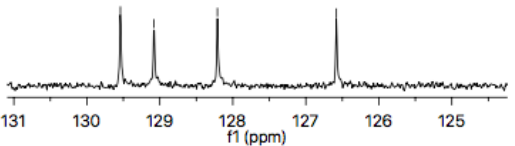

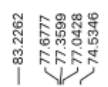

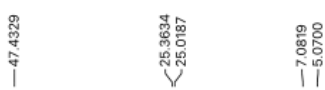
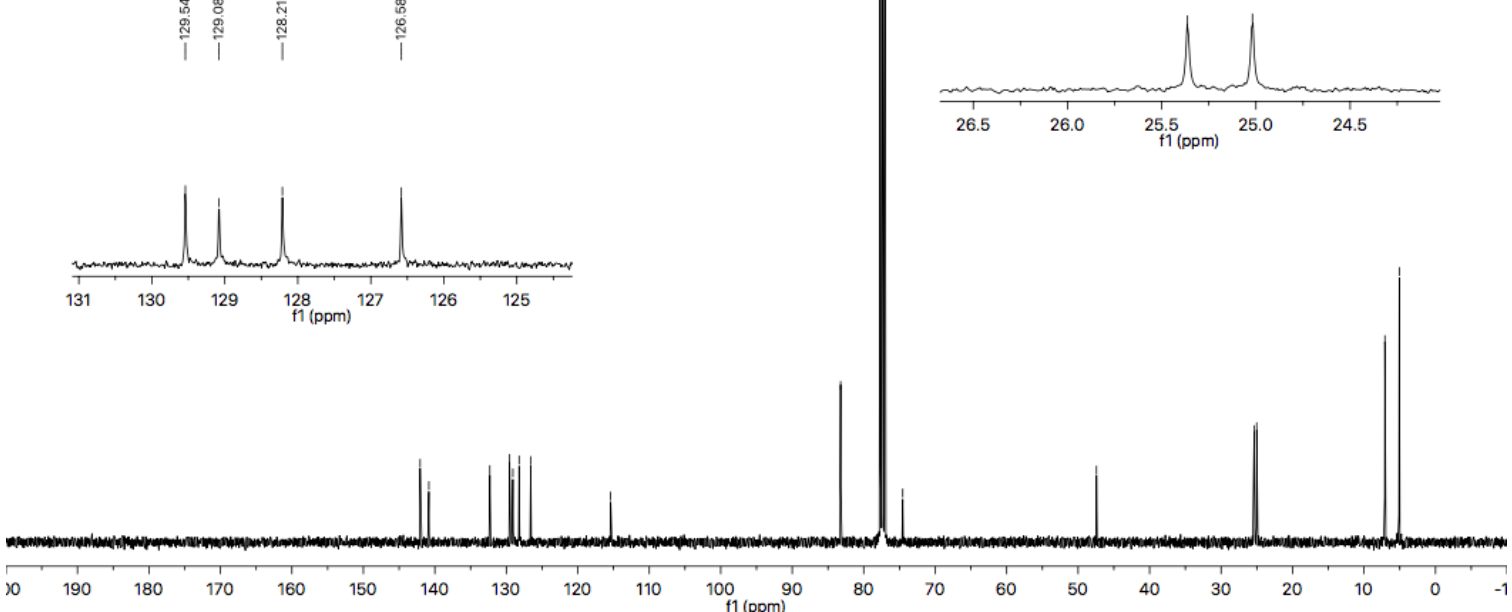

SI-50 

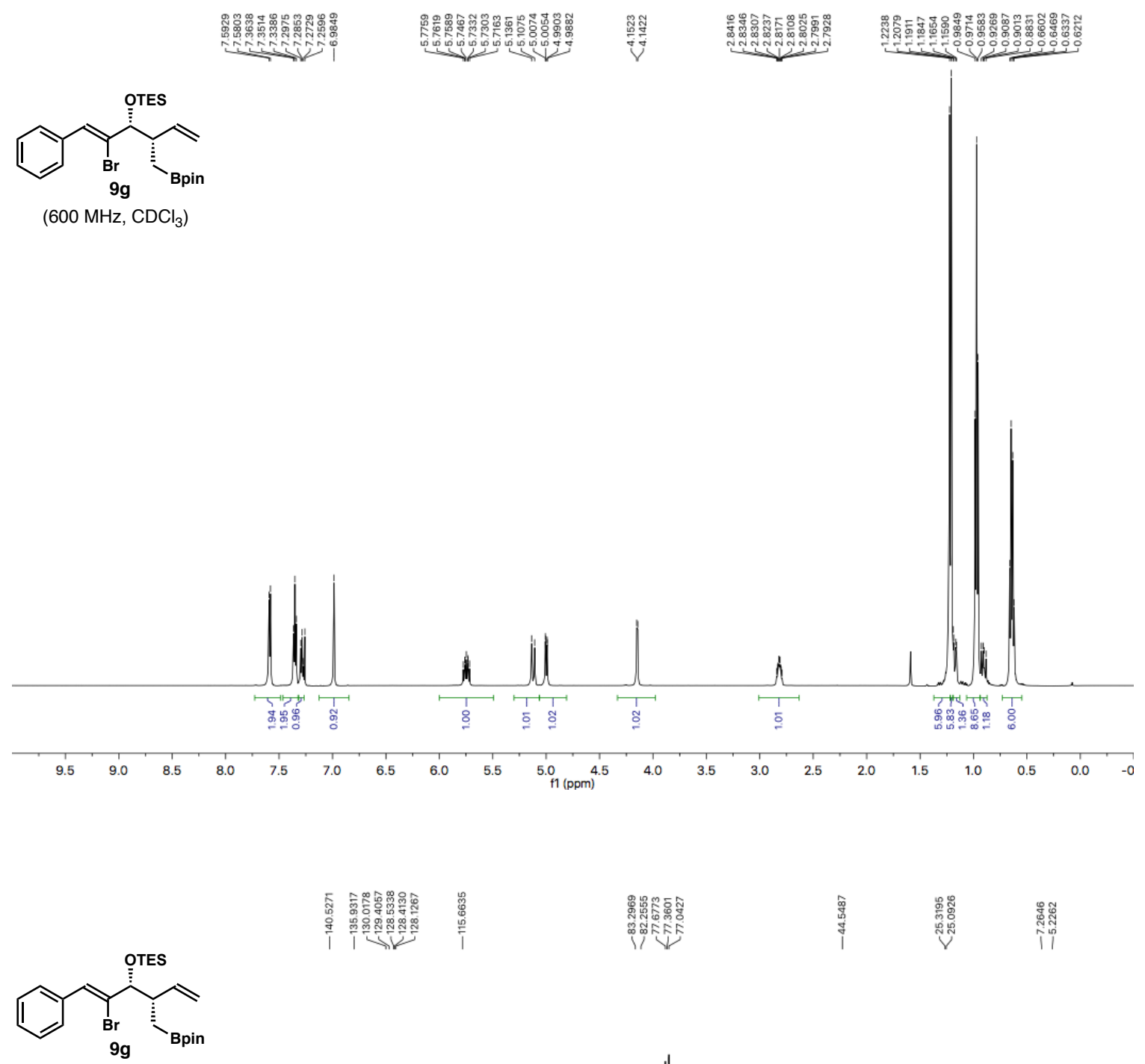

(101 MHz, $\mathrm{CDCl}_{3}$ )
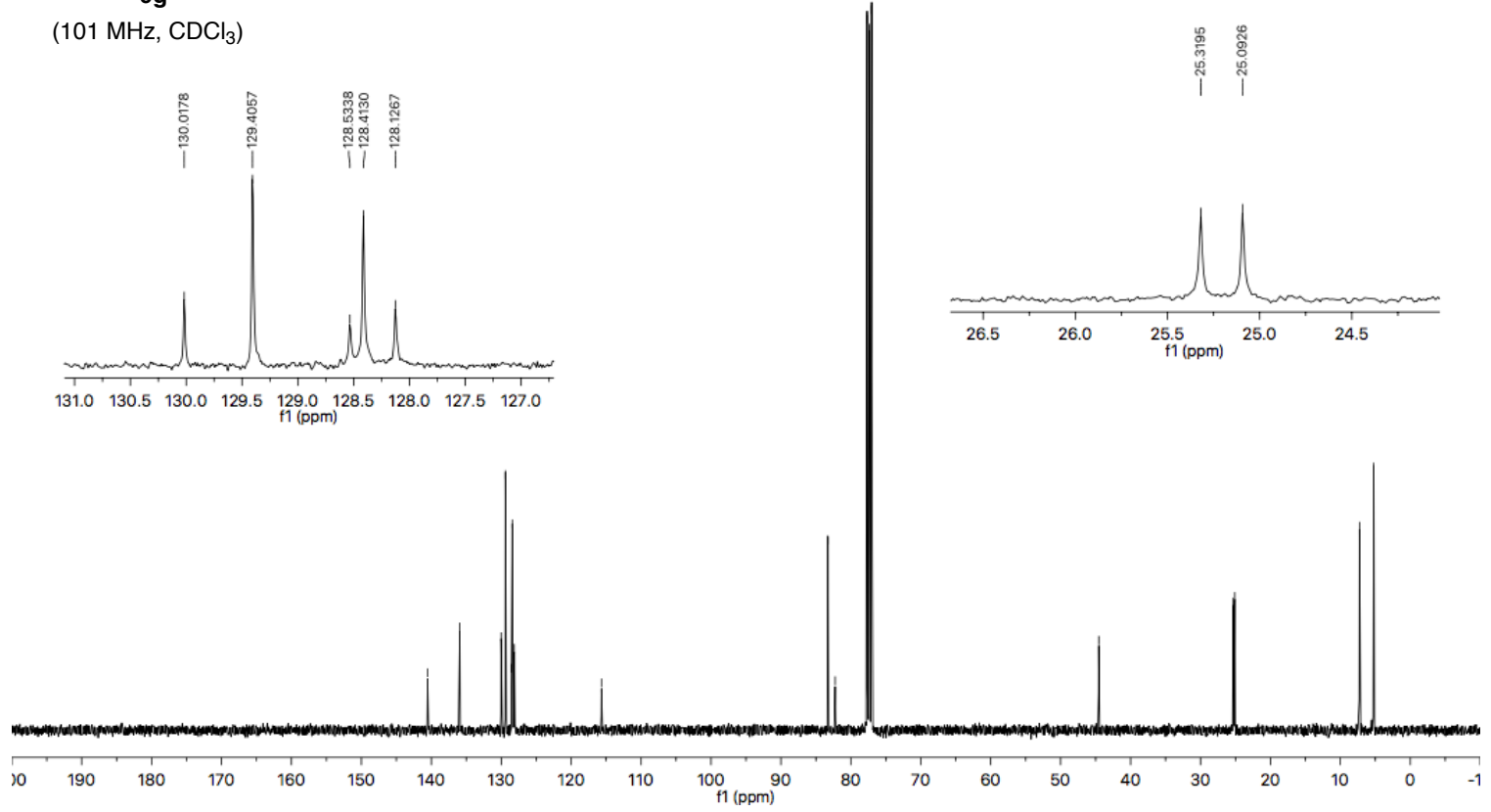

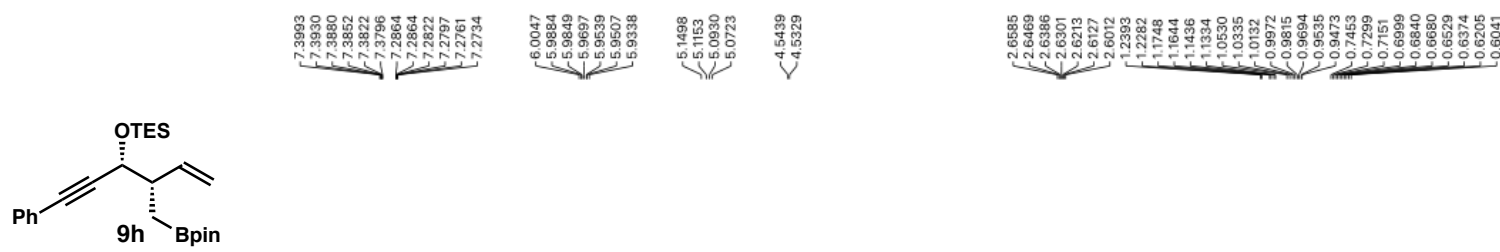

$\left(500 \mathrm{MHz}, \mathrm{CDCl}_{3}\right.$ )
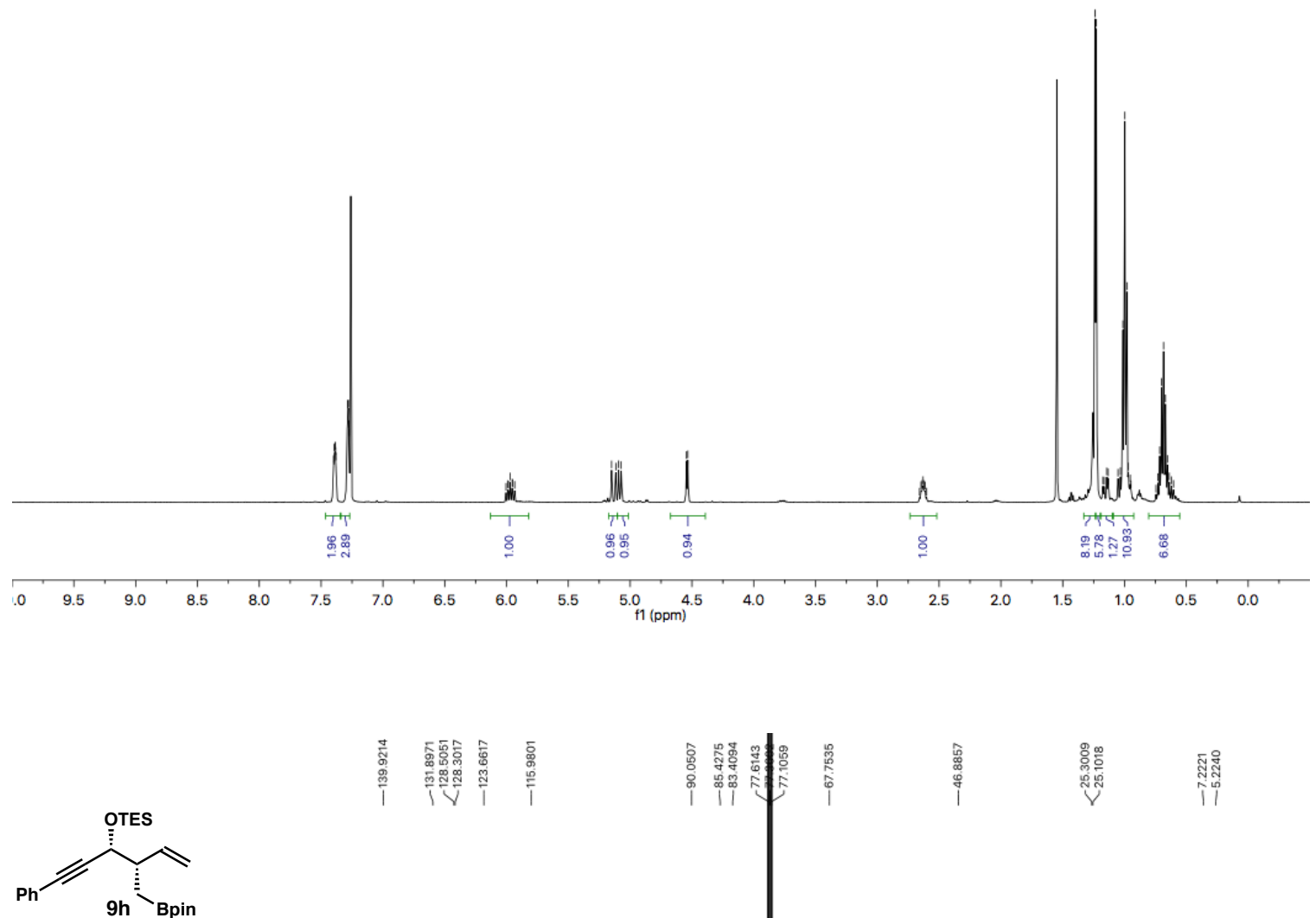

(126 MHz, $\mathrm{CDCl}_{3}$ )
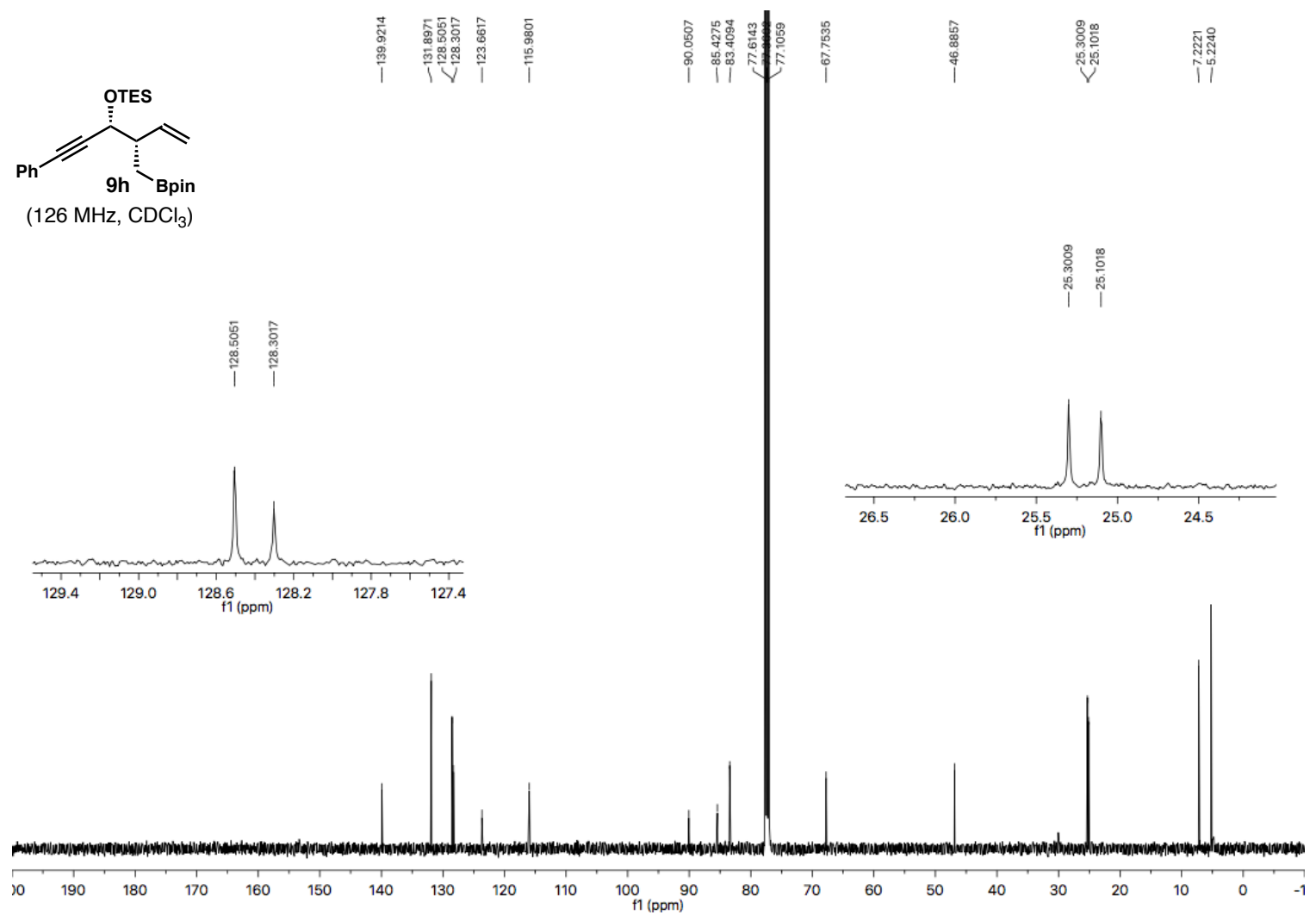

क

\section{ลูำ}



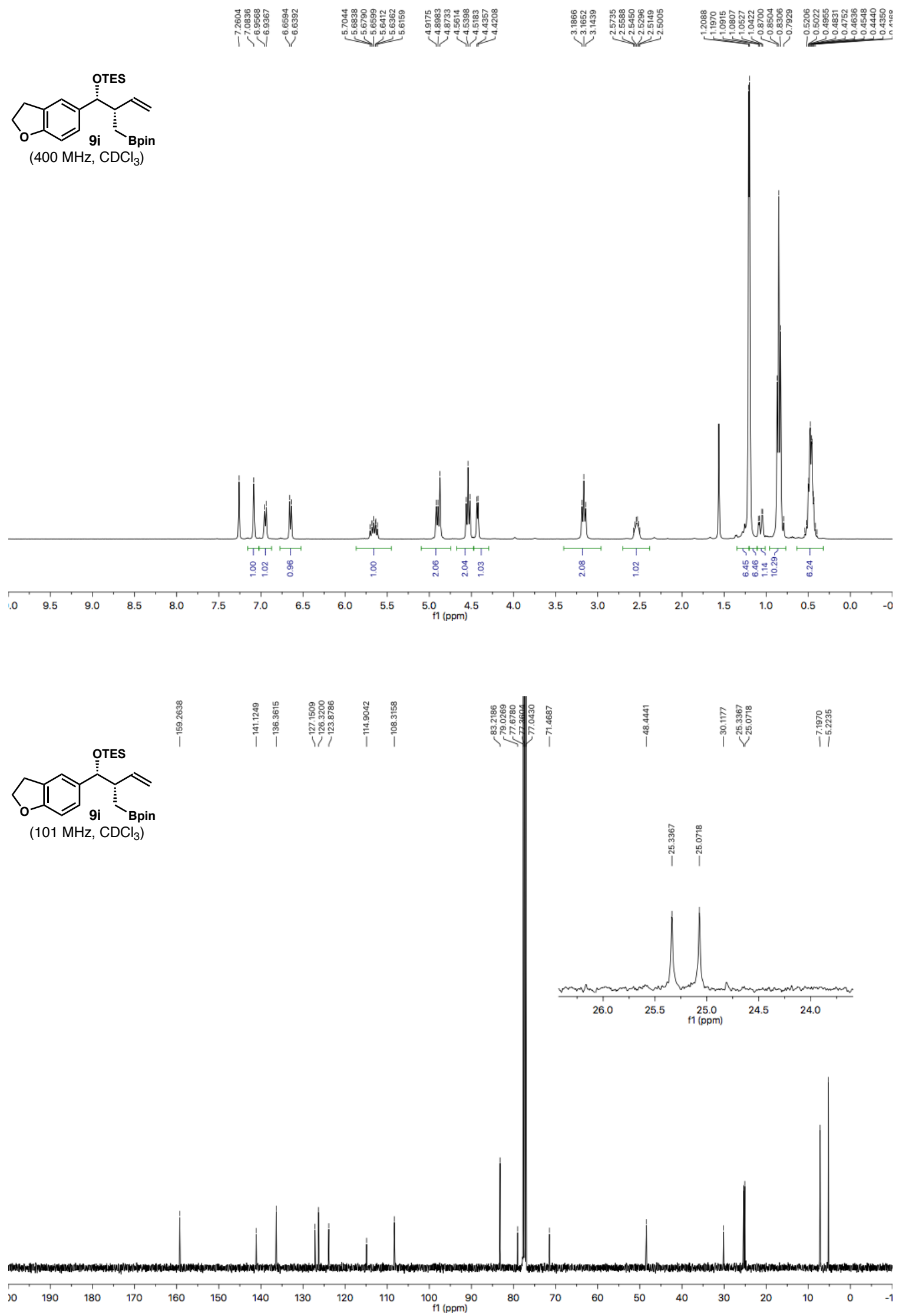

SI-53 

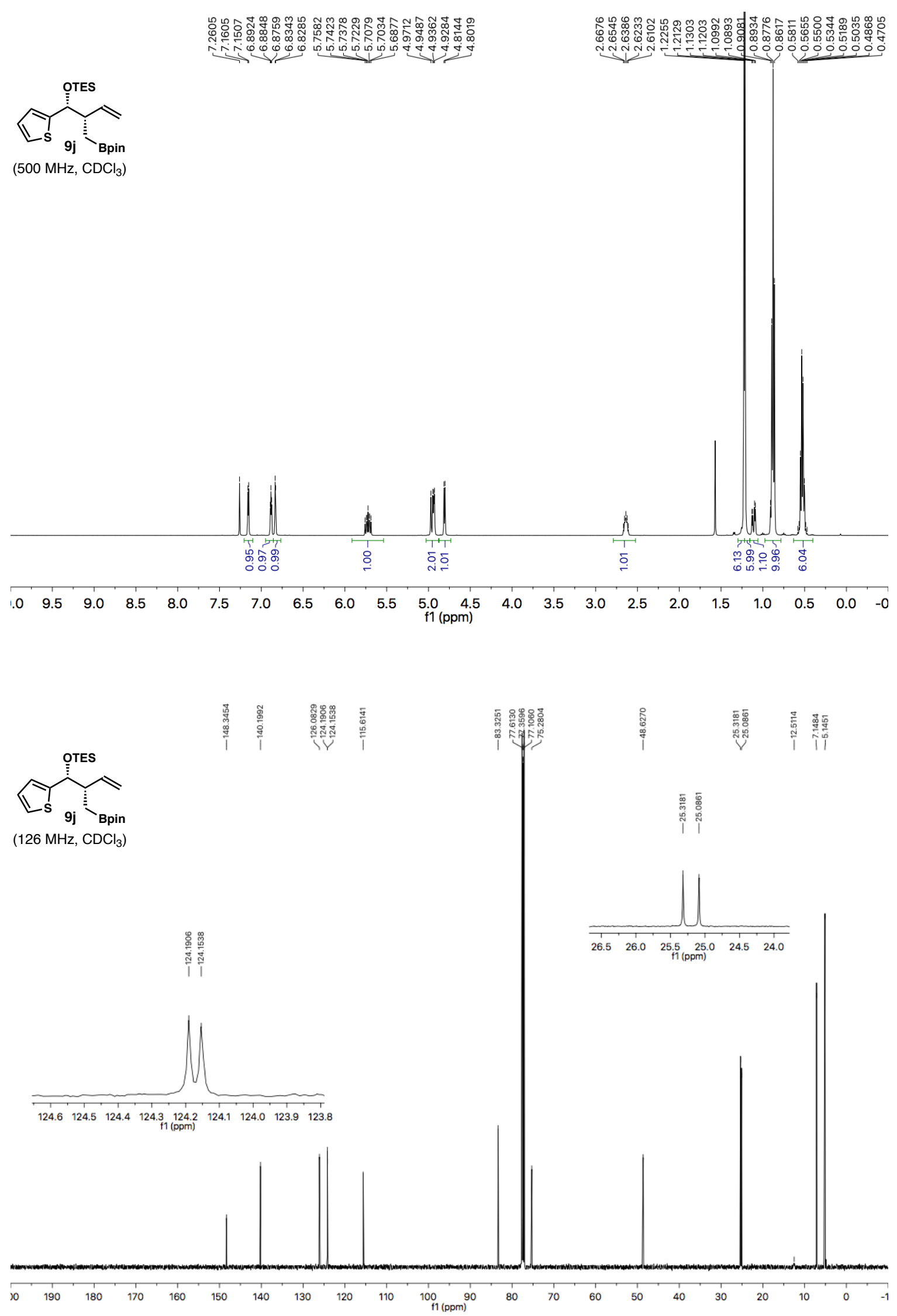

SI-54 

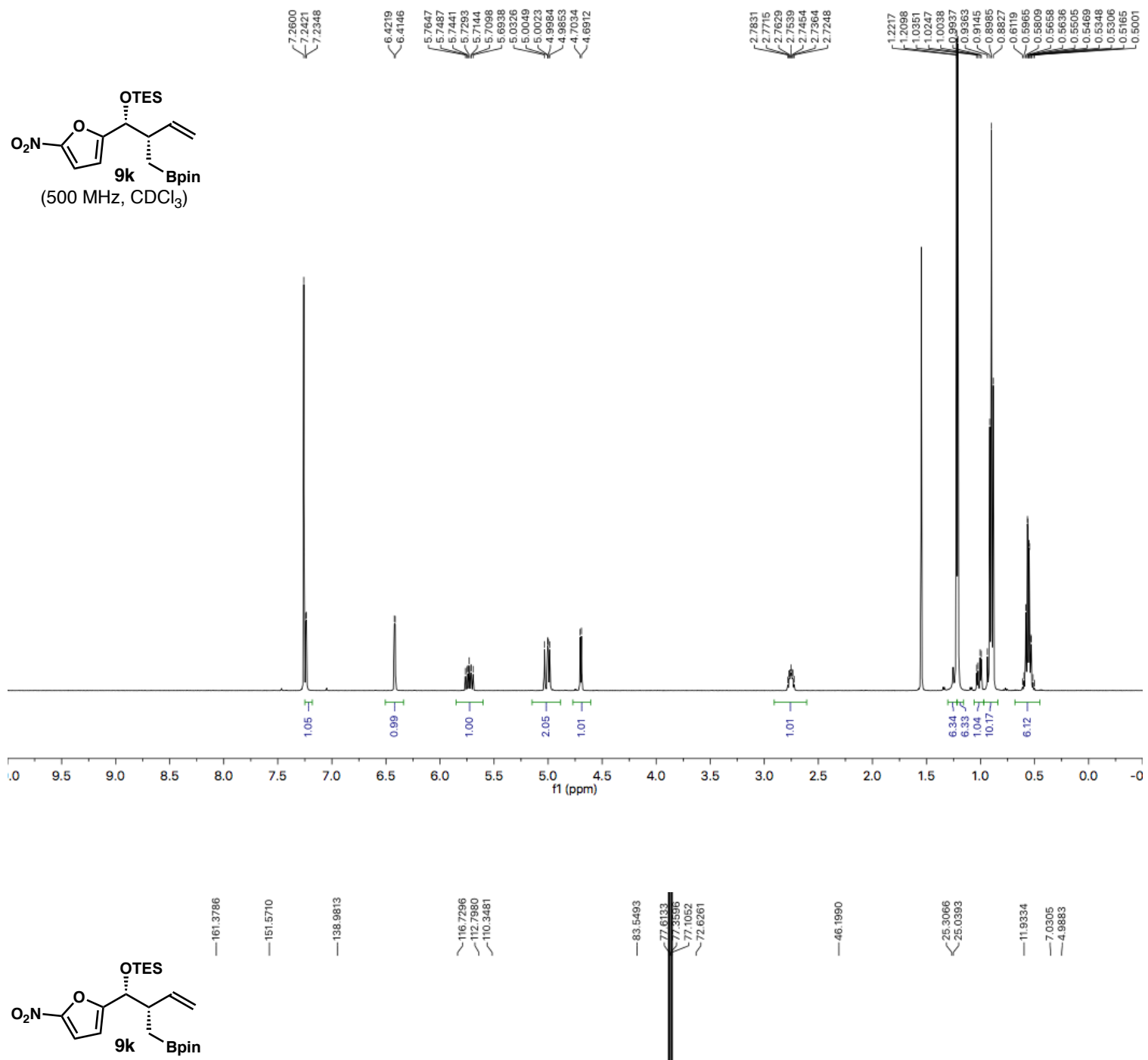

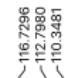

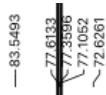

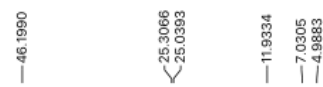

(126 MHz, $\mathrm{CDCl}_{3}$ )
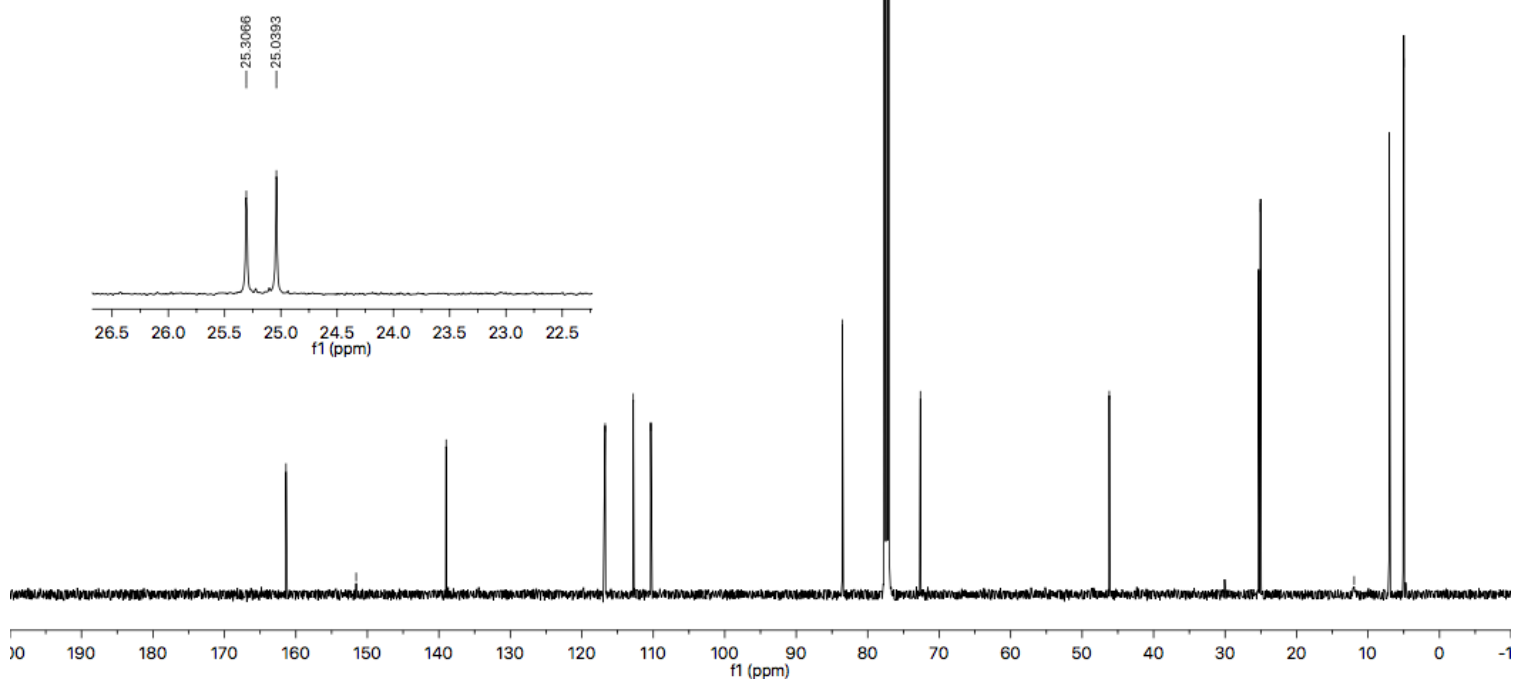

SI-55 

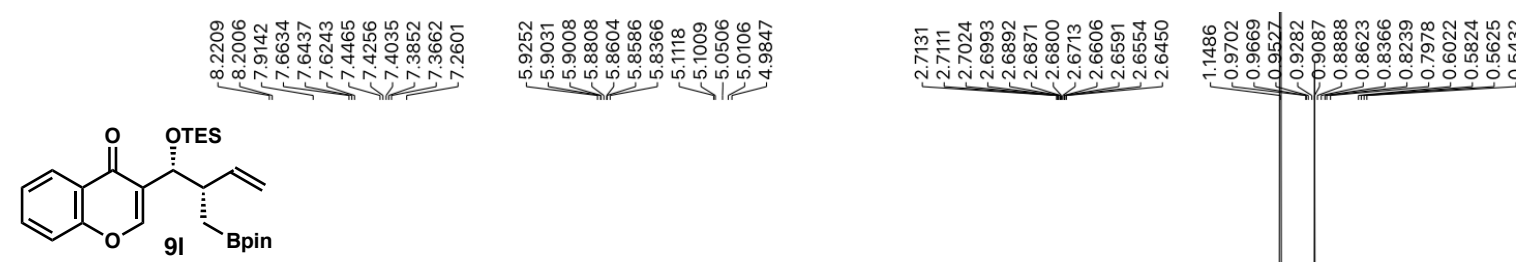

$\left(400 \mathrm{MHz}, \mathrm{CDCl}_{3}\right.$ )
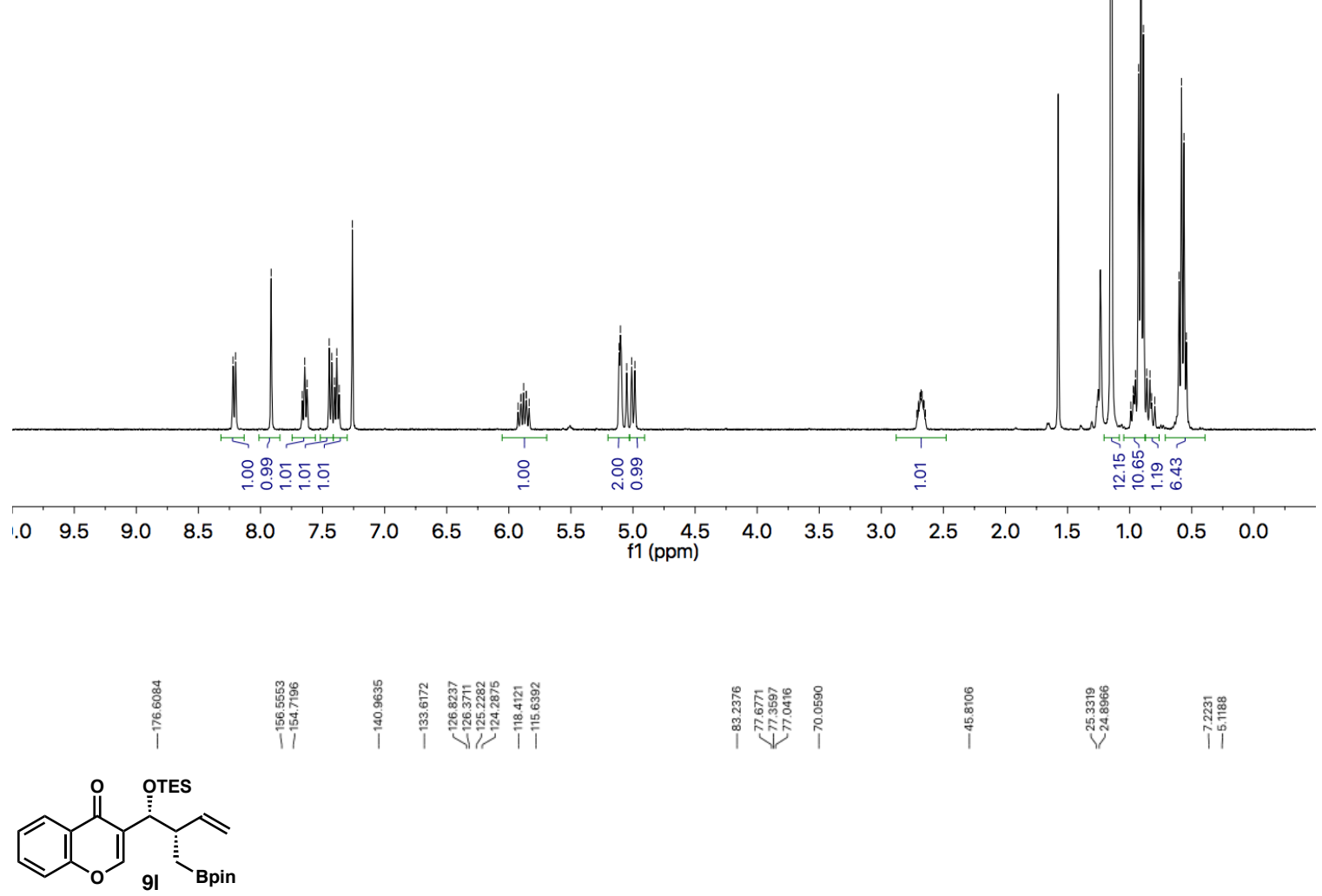

$\left(101 \mathrm{MHz}, \mathrm{CDCl}_{3}\right.$ )
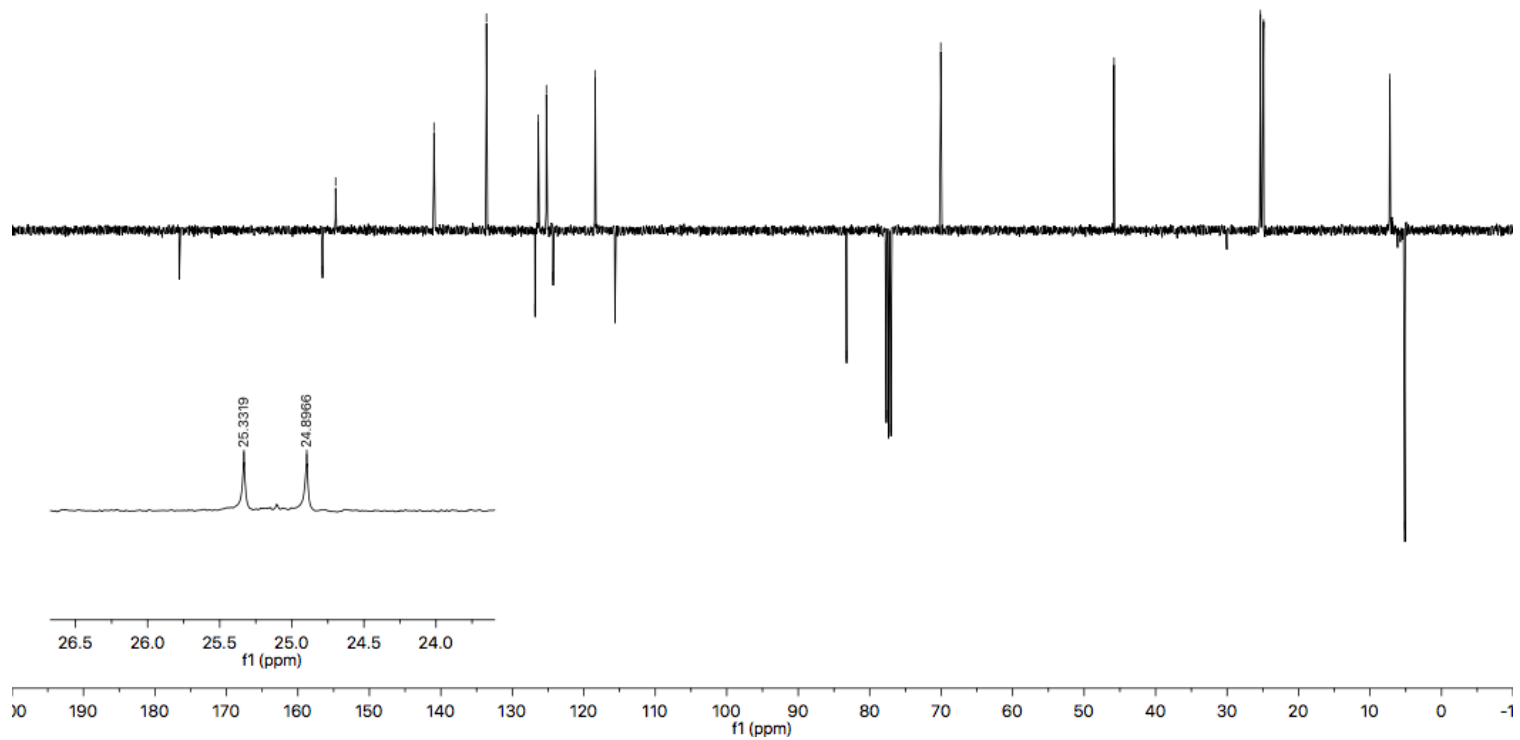


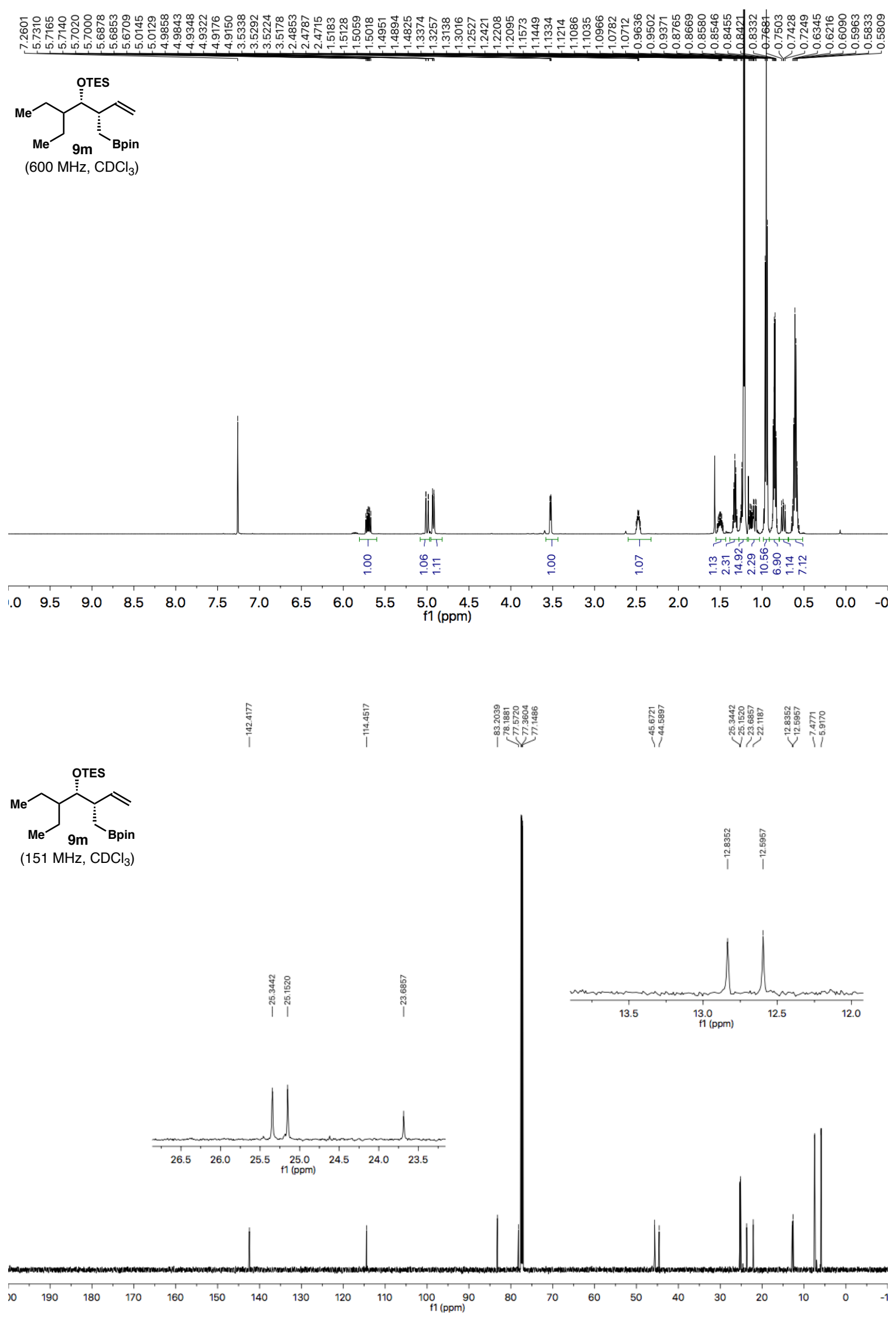

SI-57 

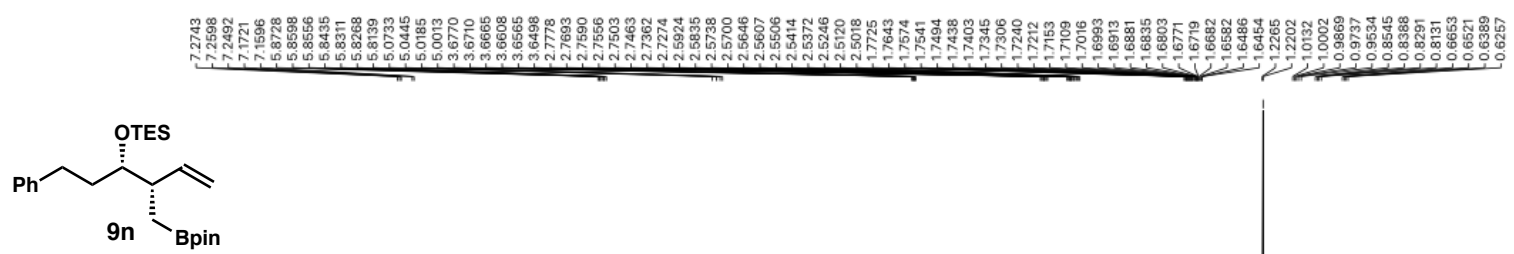

(600 MHz, $\mathrm{CDCl}_{3}$ )
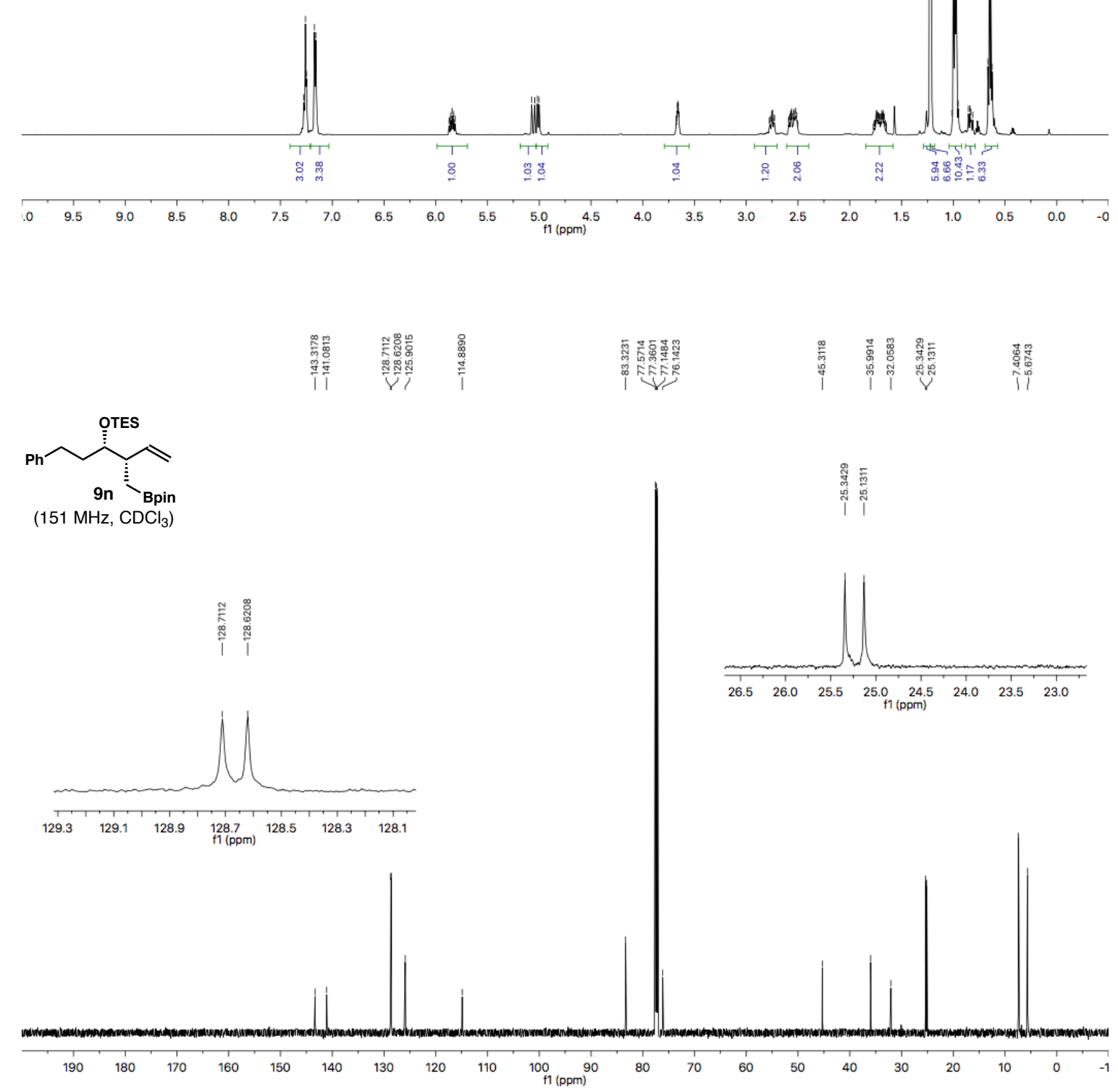

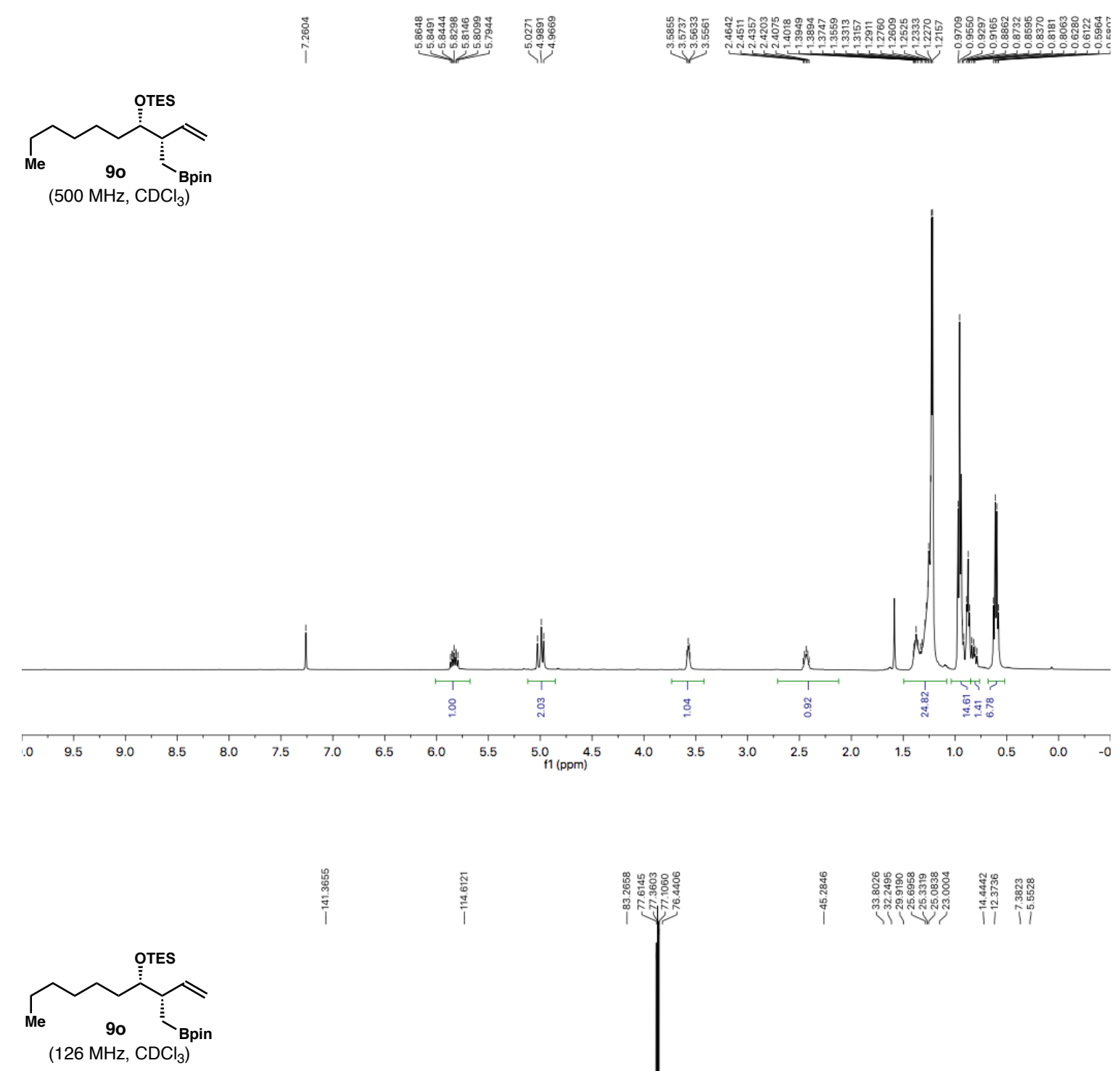

竞
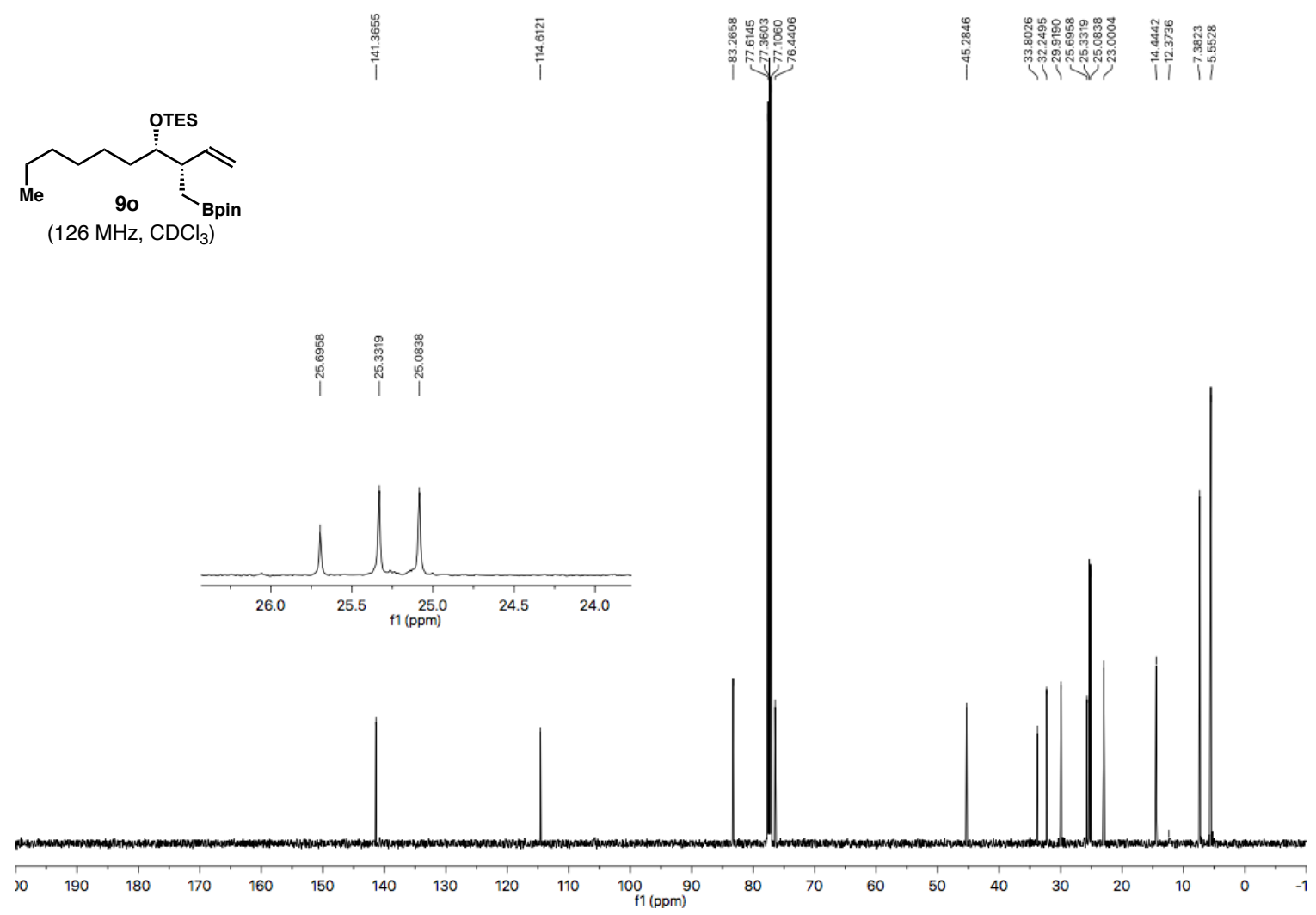

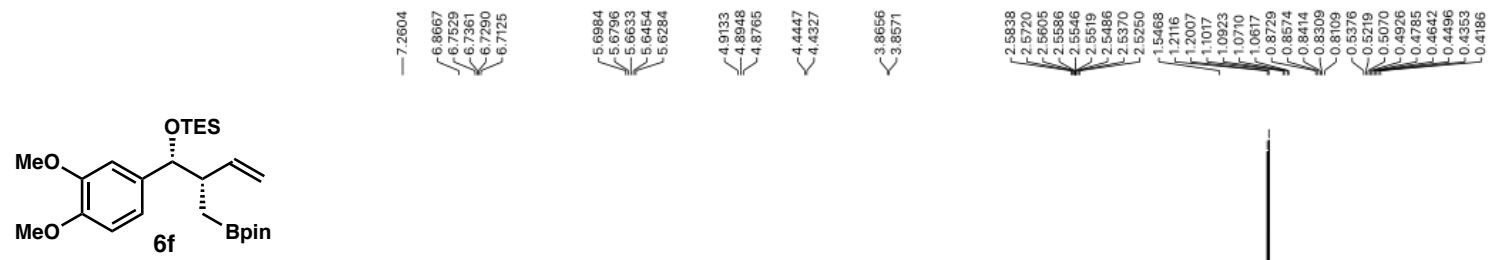

$\left(500 \mathrm{MHz}, \mathrm{CDCl}_{3}\right.$ )
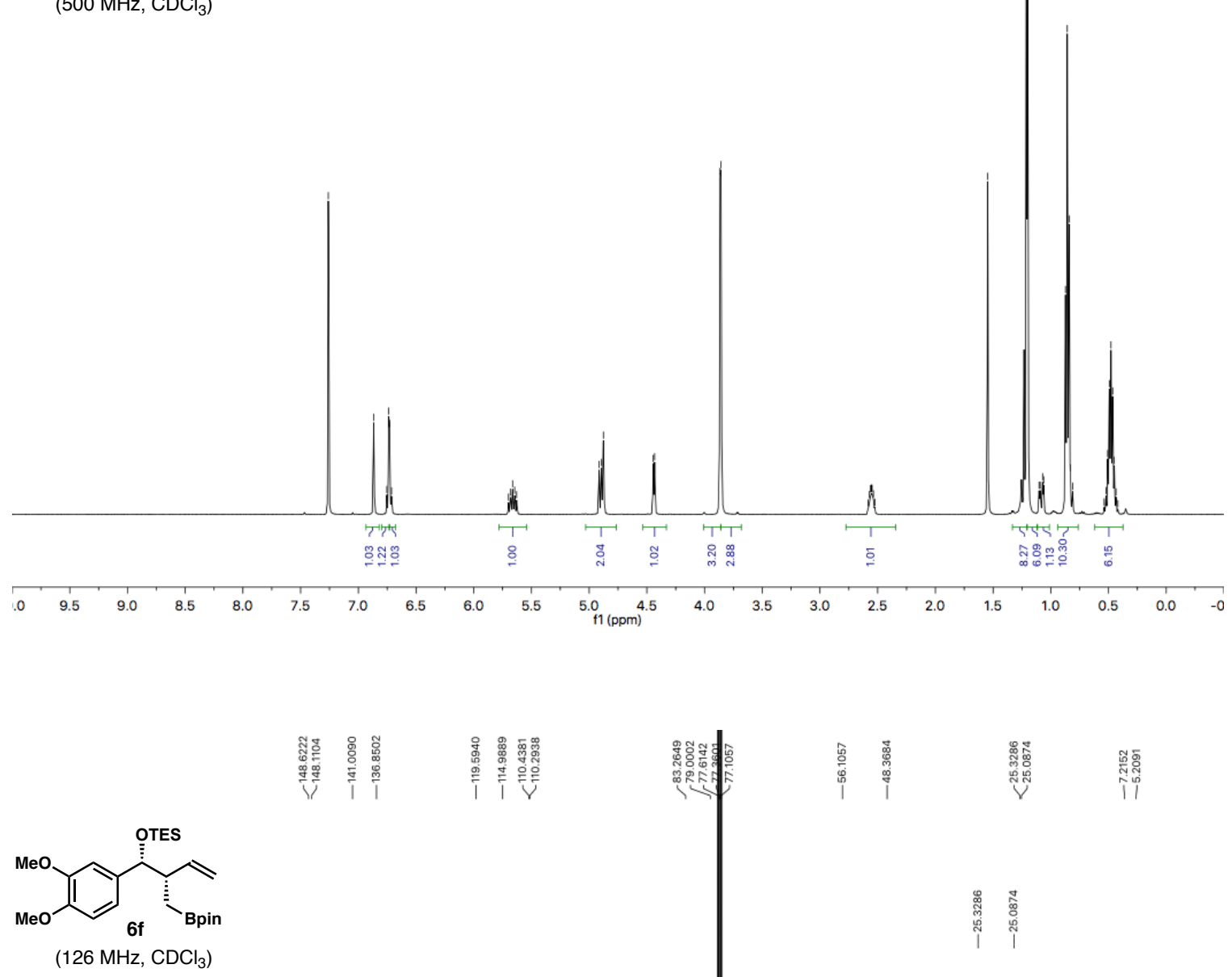

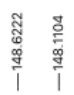
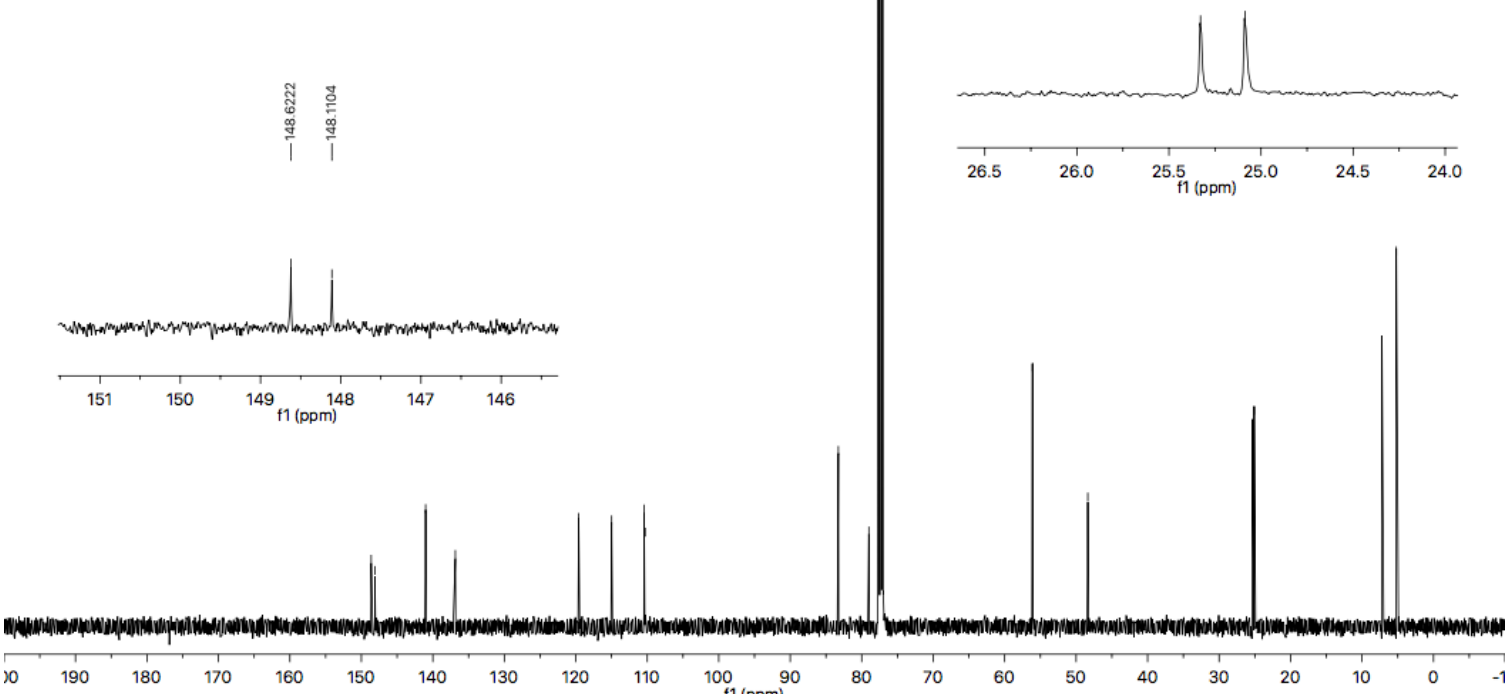

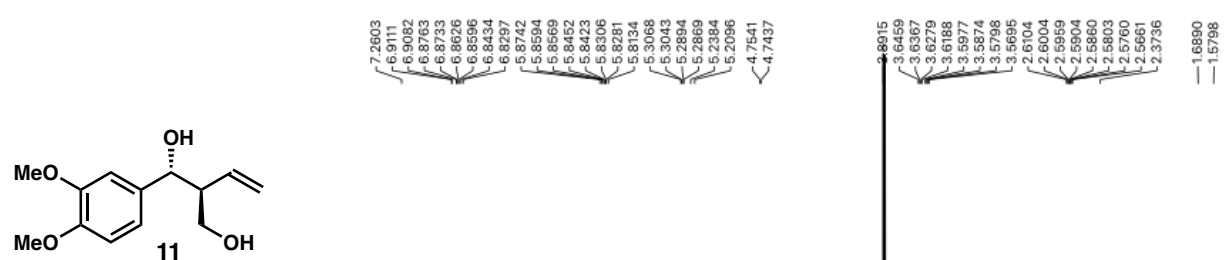

$\left(600 \mathrm{MHz}, \mathrm{CDCl}_{3}\right.$ )

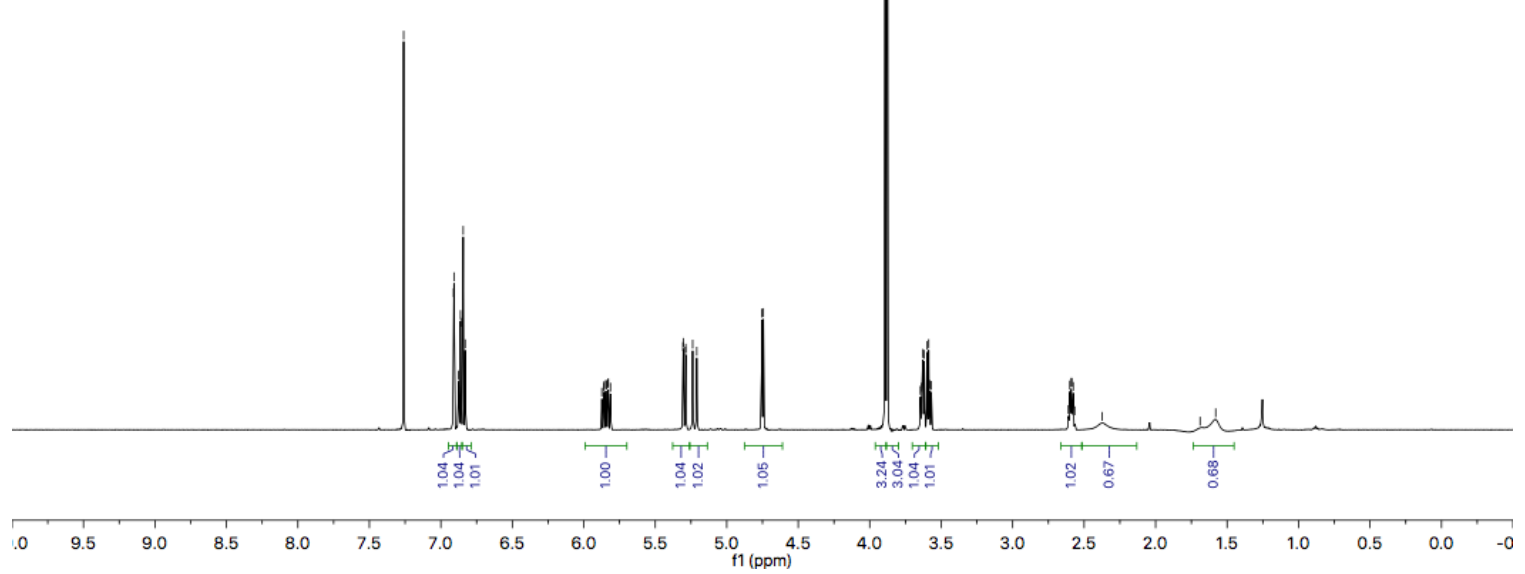

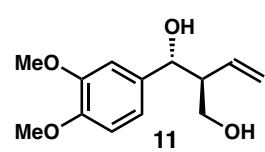

$\left(126 \mathrm{MHz}, \mathrm{CDCl}_{3}\right.$ )

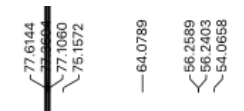

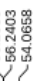

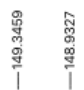

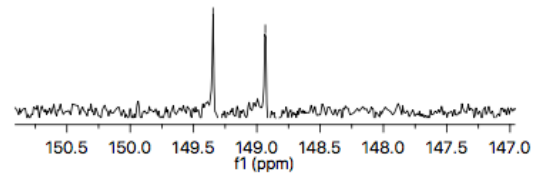

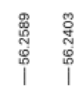

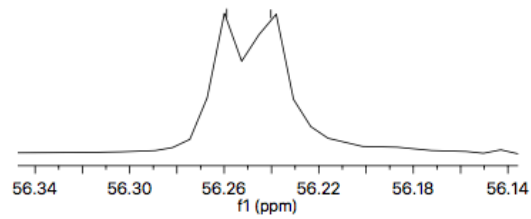
$\begin{array}{llllllll}150.5 & 150.0 & 149.5 & 149.0 & 148.5 & 148.0 & 147.5 & 147.0\end{array}$

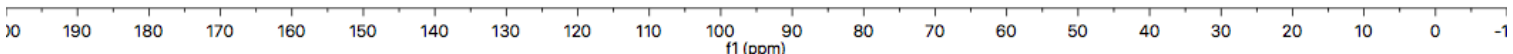



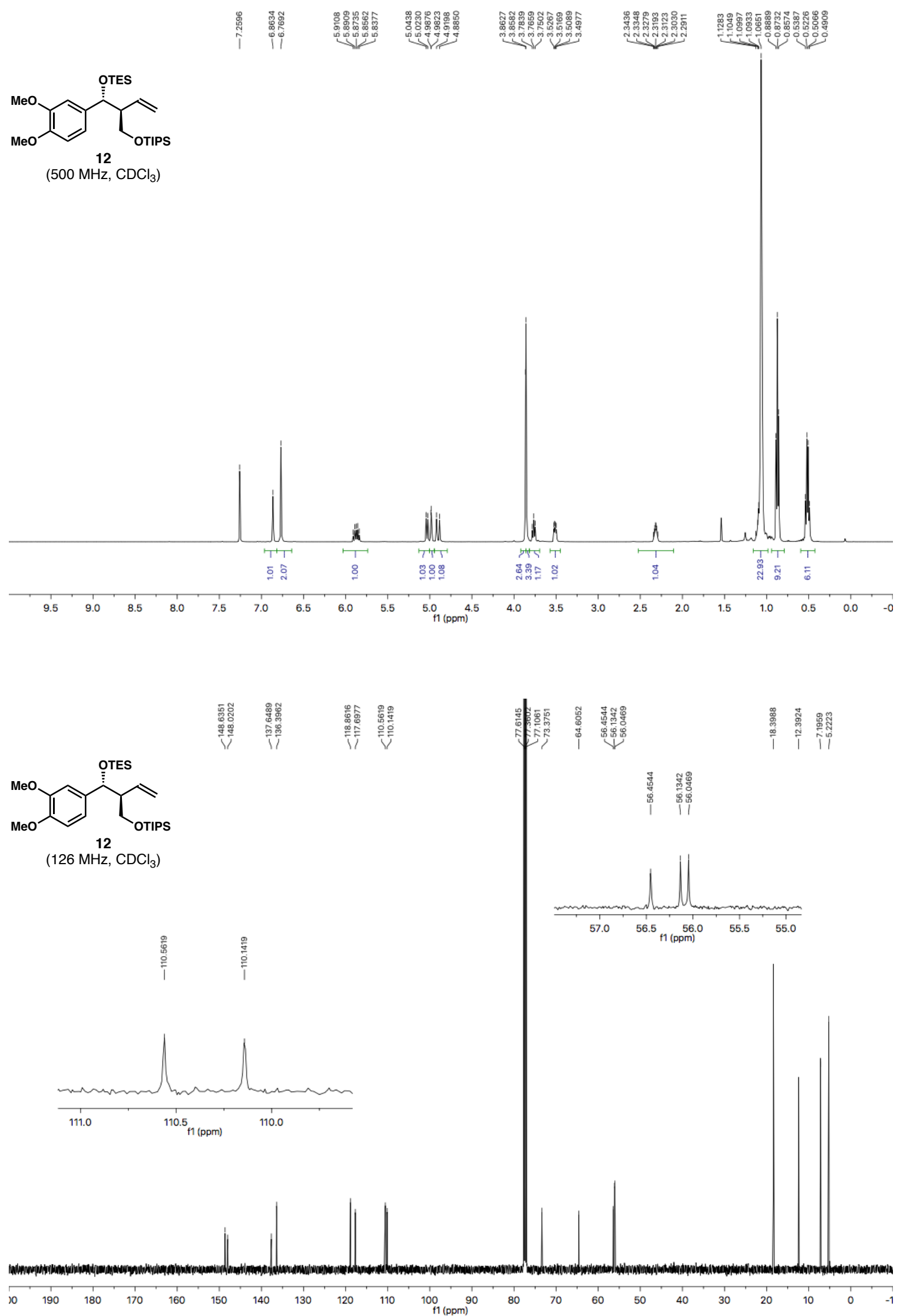

(1)

SI-62 

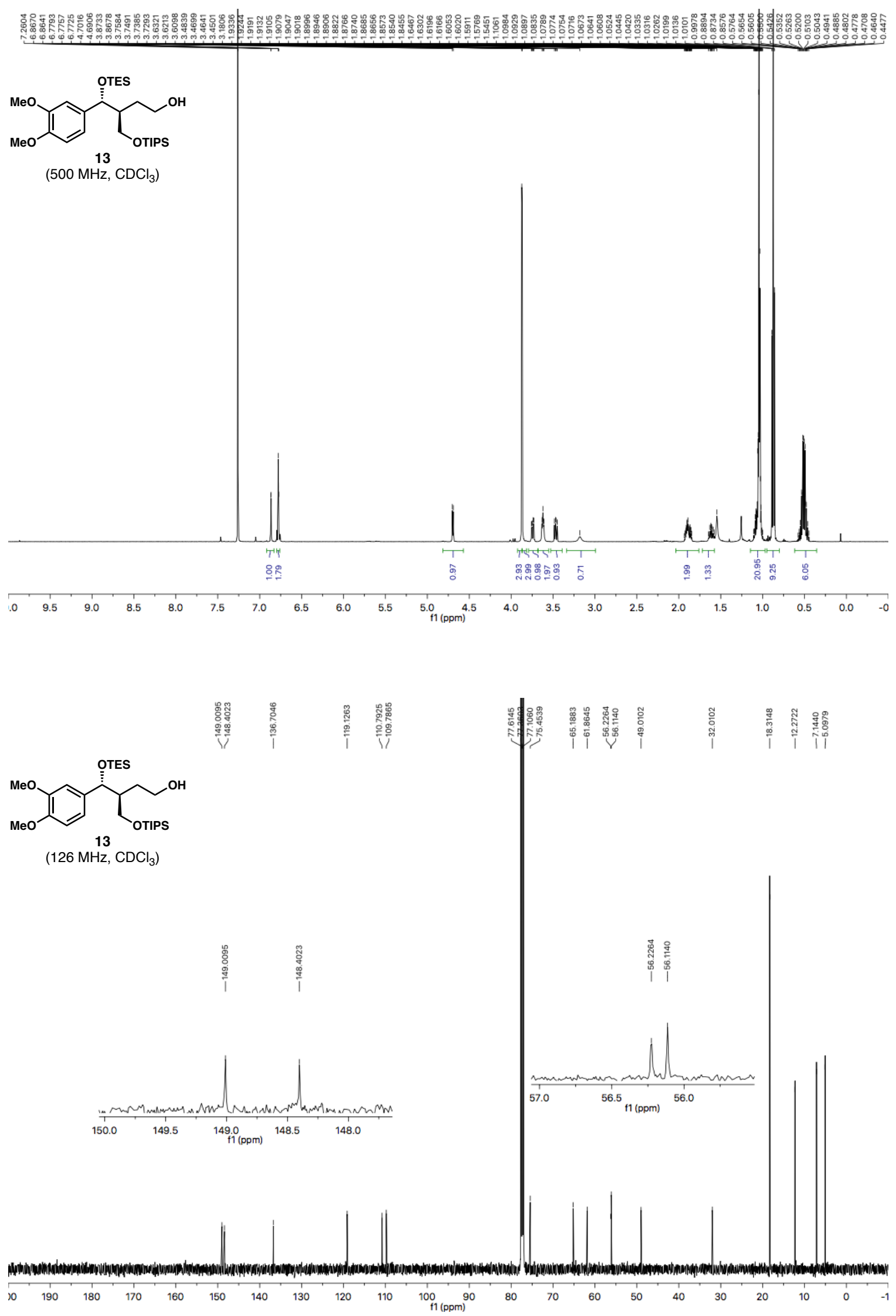

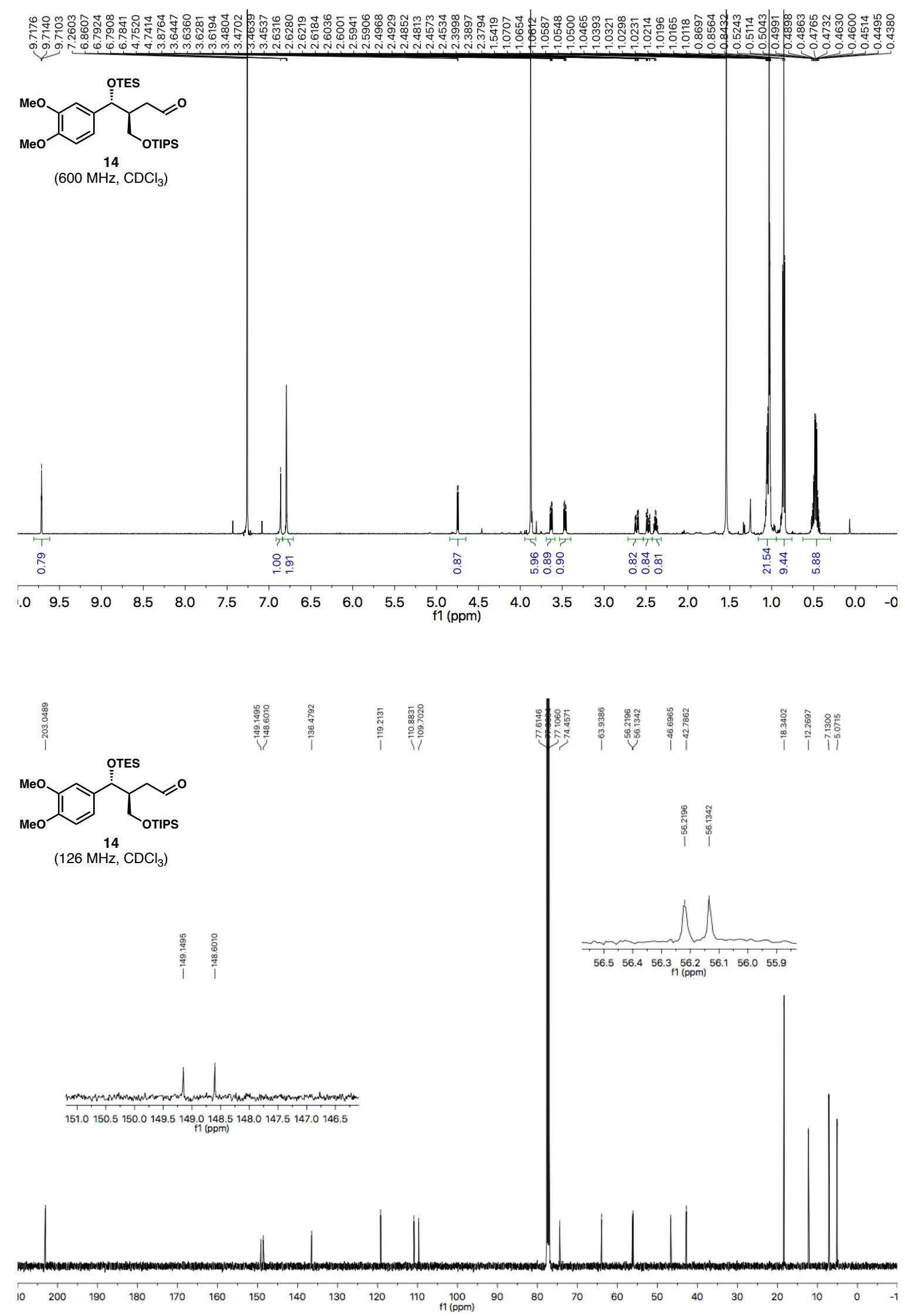

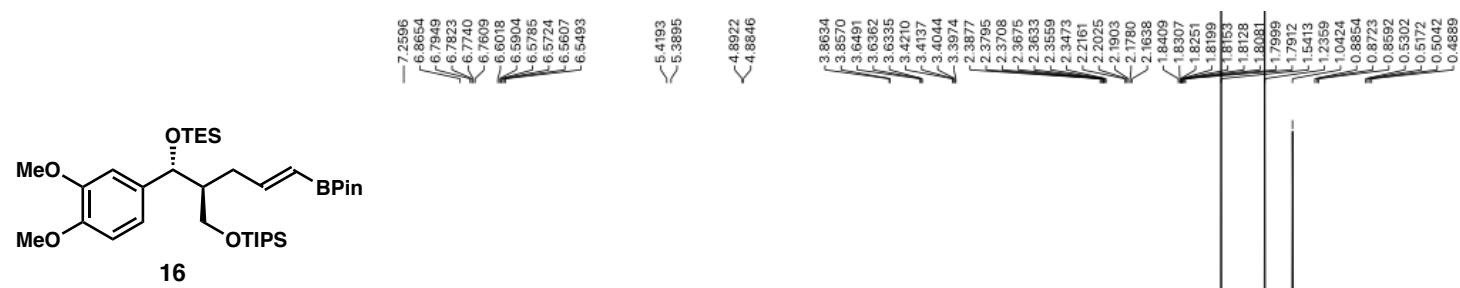

(600 MHz, $\mathrm{CDCl}_{3}$ )
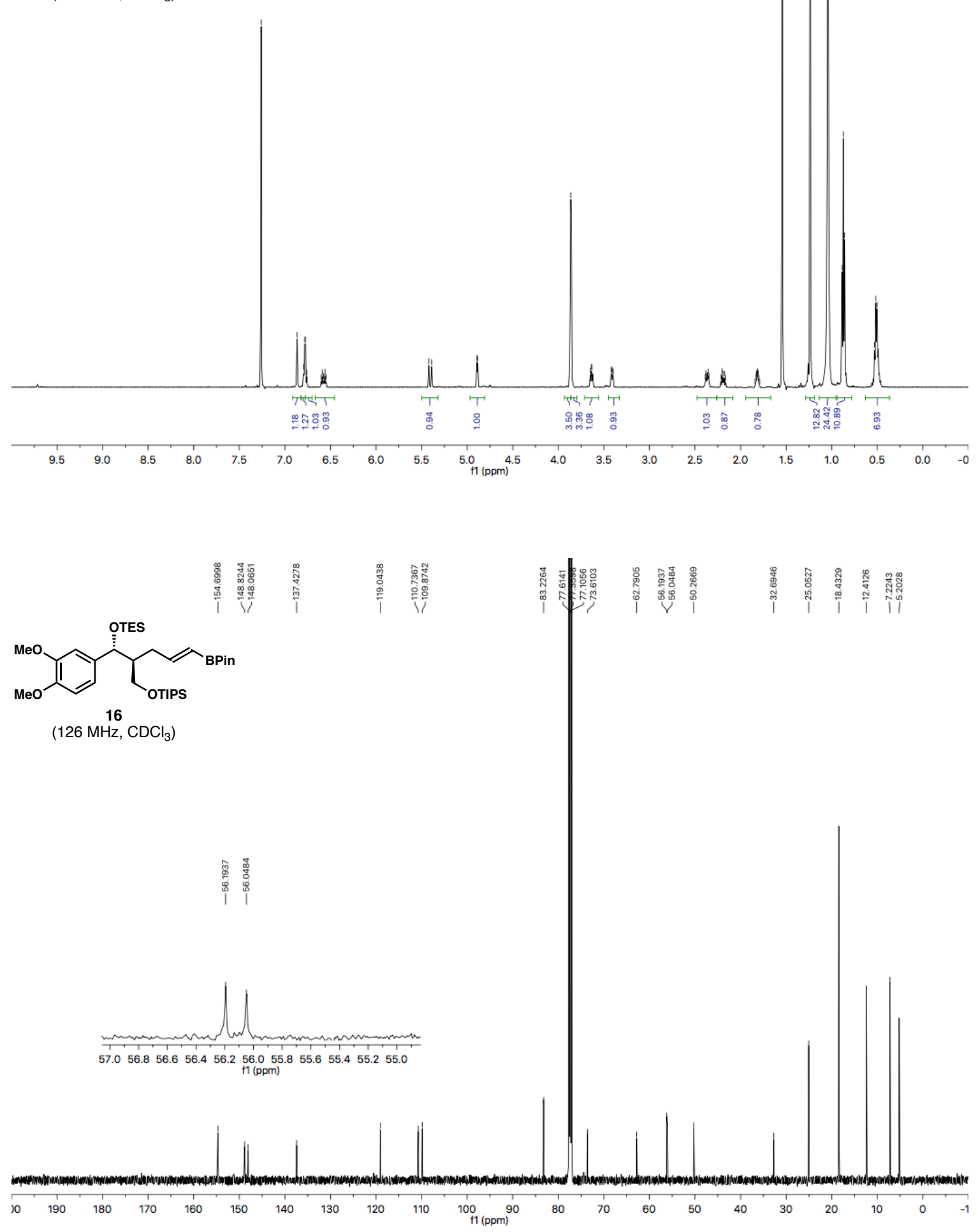

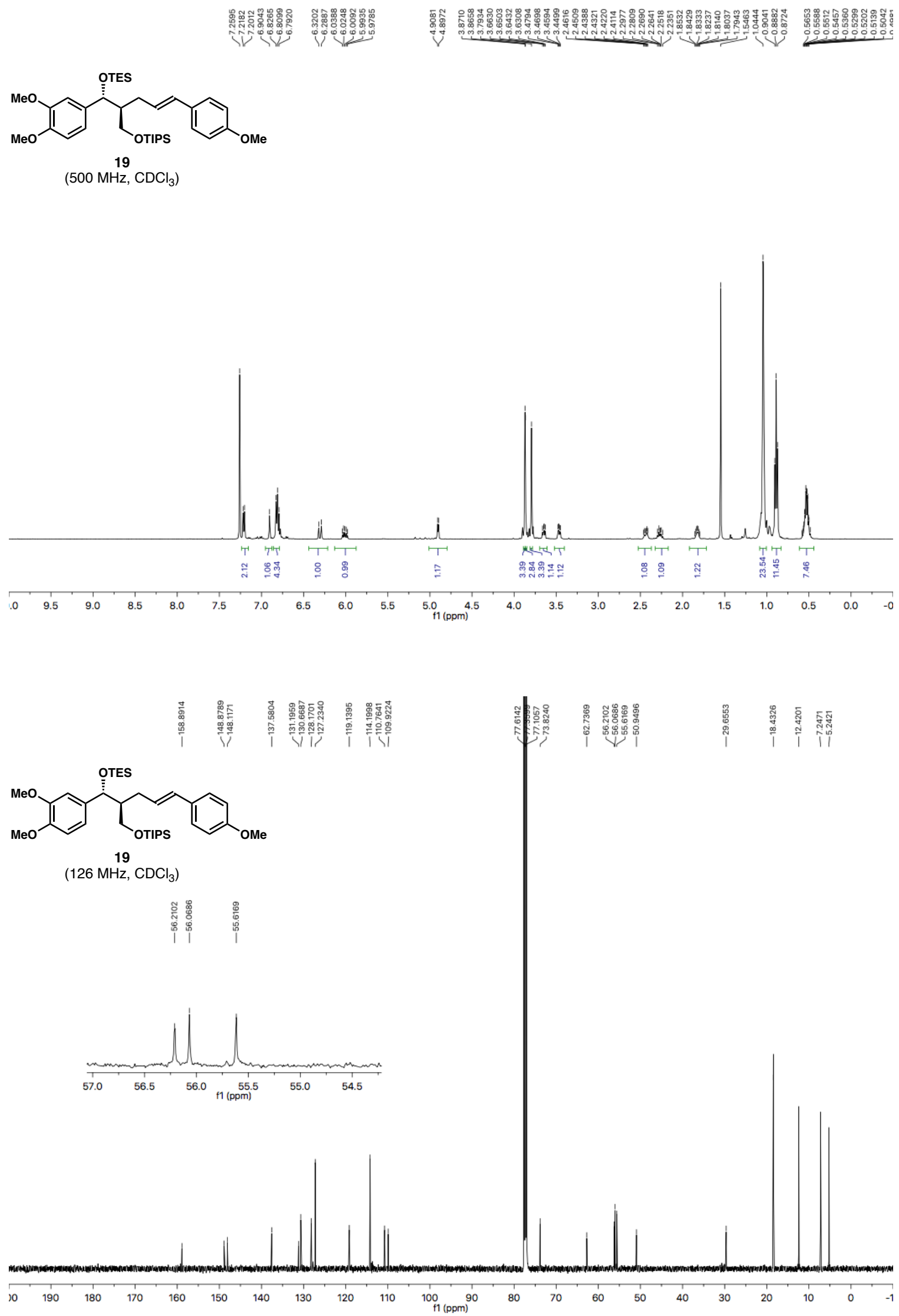

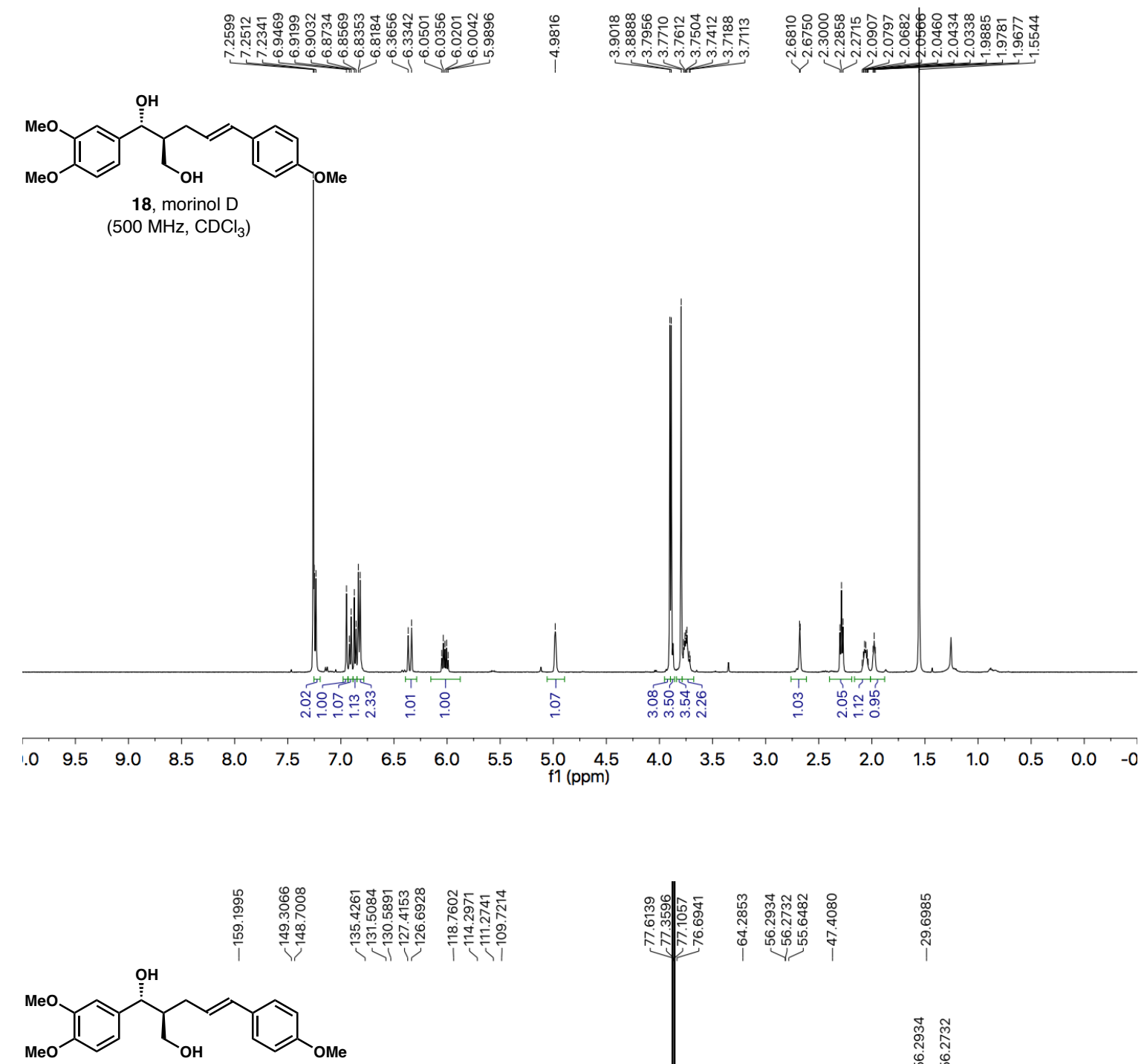

18, morinol D
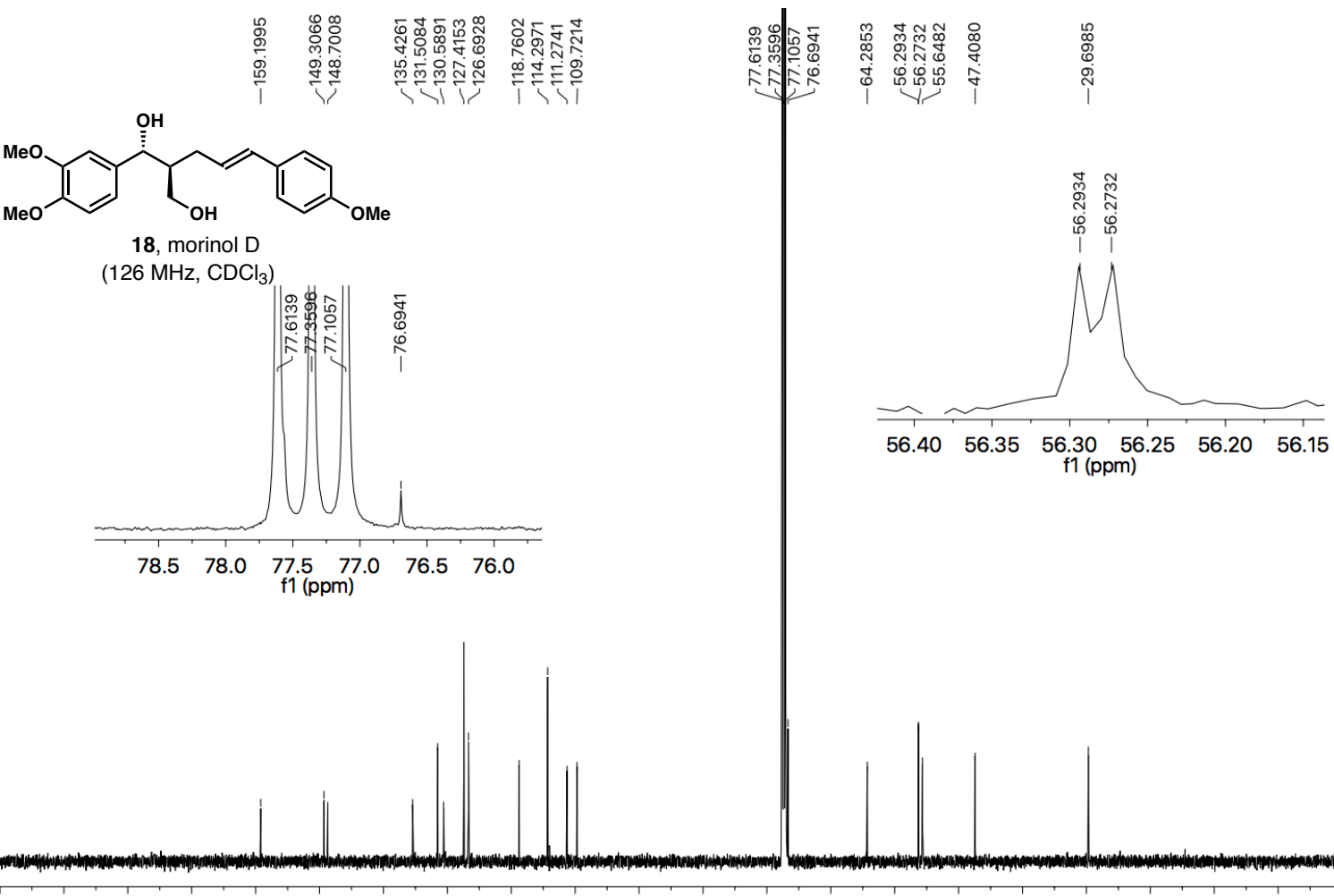

$\begin{array}{lllllllllllllllllllll}10 & 190 & 180 & 170 & 160 & 150 & 140 & 130 & 120 & 110 & 100 & 90 & 80 & 70 & 60 & 50 & 40 & 30 & 20 & 10 & 0\end{array}$ 

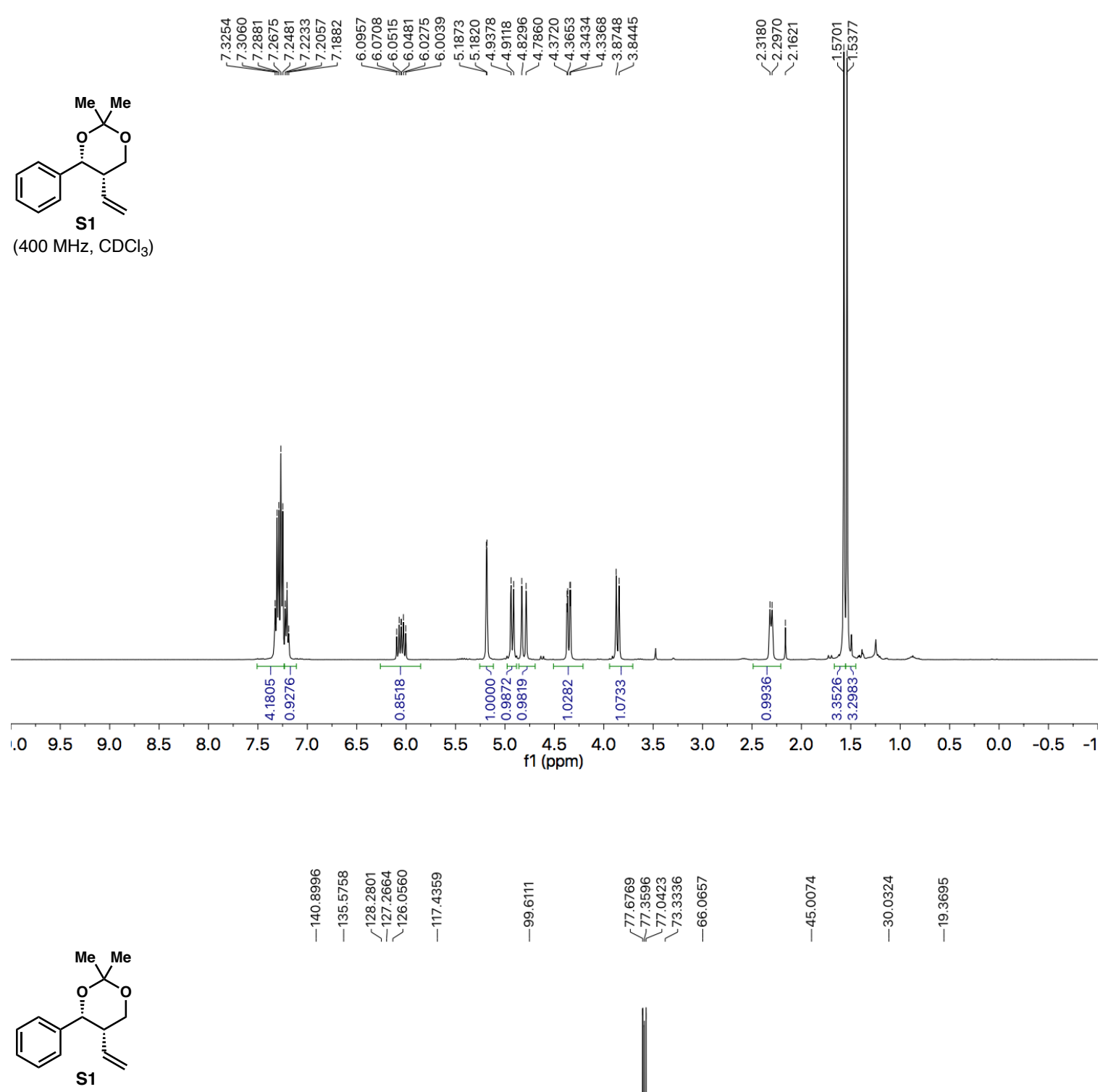

(101 MHz, $\mathrm{CDCl}_{3}$ )

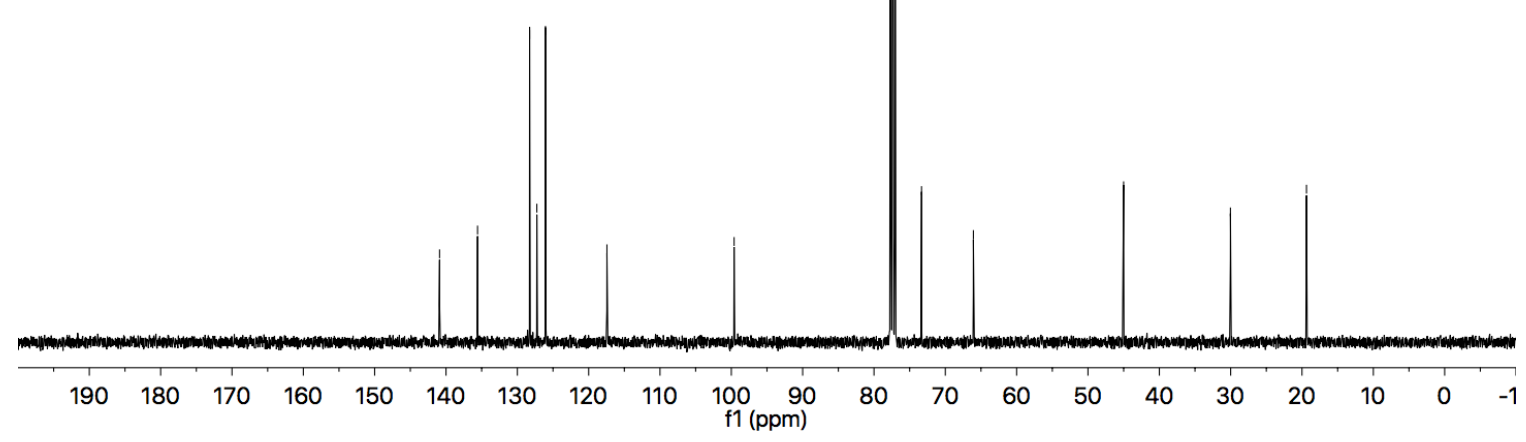



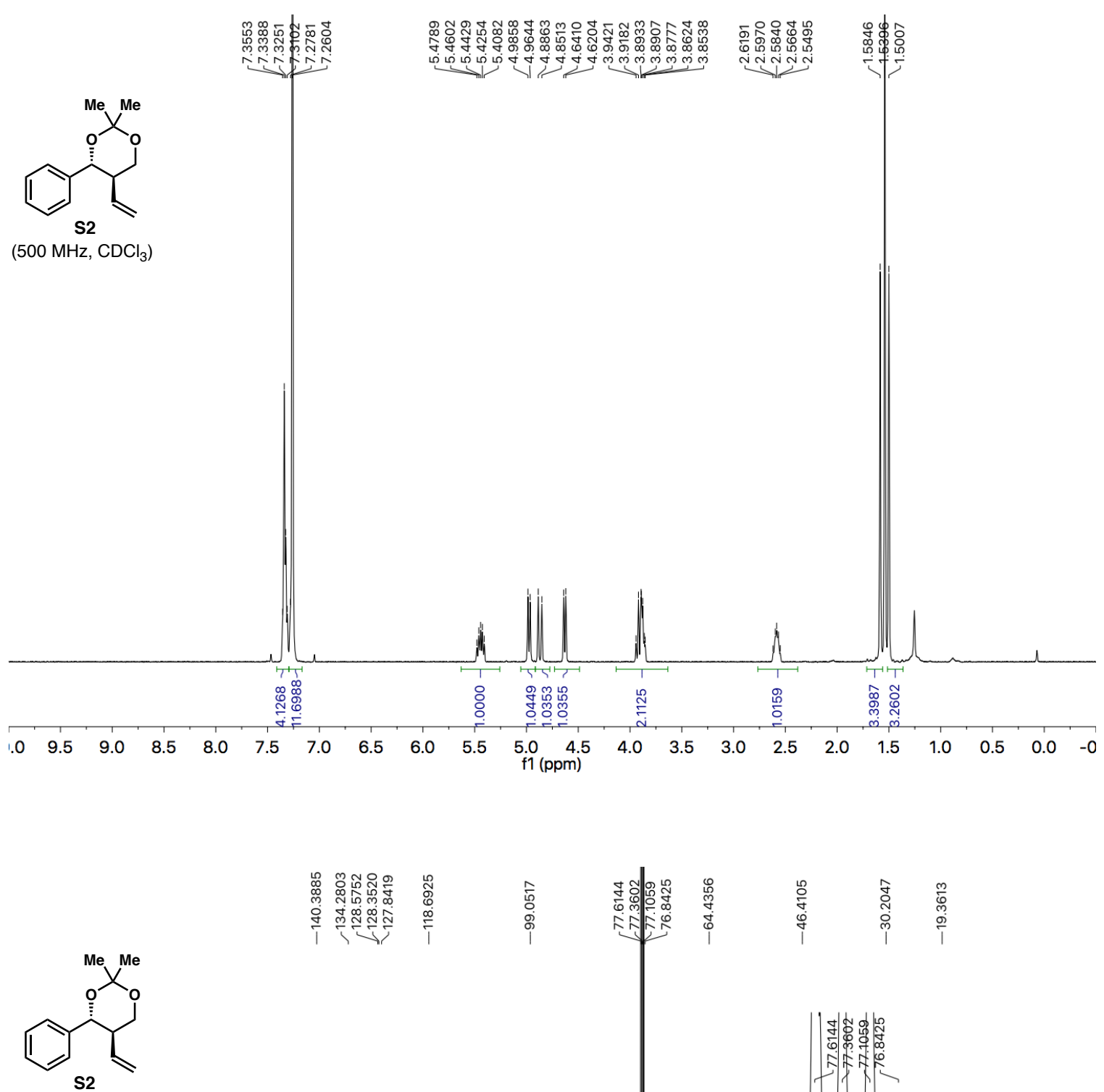

(126 MHz, $\mathrm{CDCl}_{3}$ )
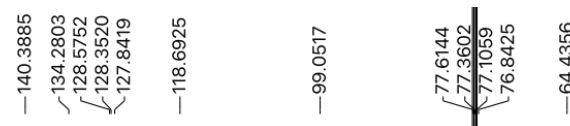

$\begin{array}{llll}0 & 0 & 0 \\ 0 & 0 & 0 \\ 0 & 0 & 0 \\ 0 & 0 & 0 & 0 \\ 0 & 0 & 0 & 0 \\ 1 & 1 & 0 & 1\end{array}$

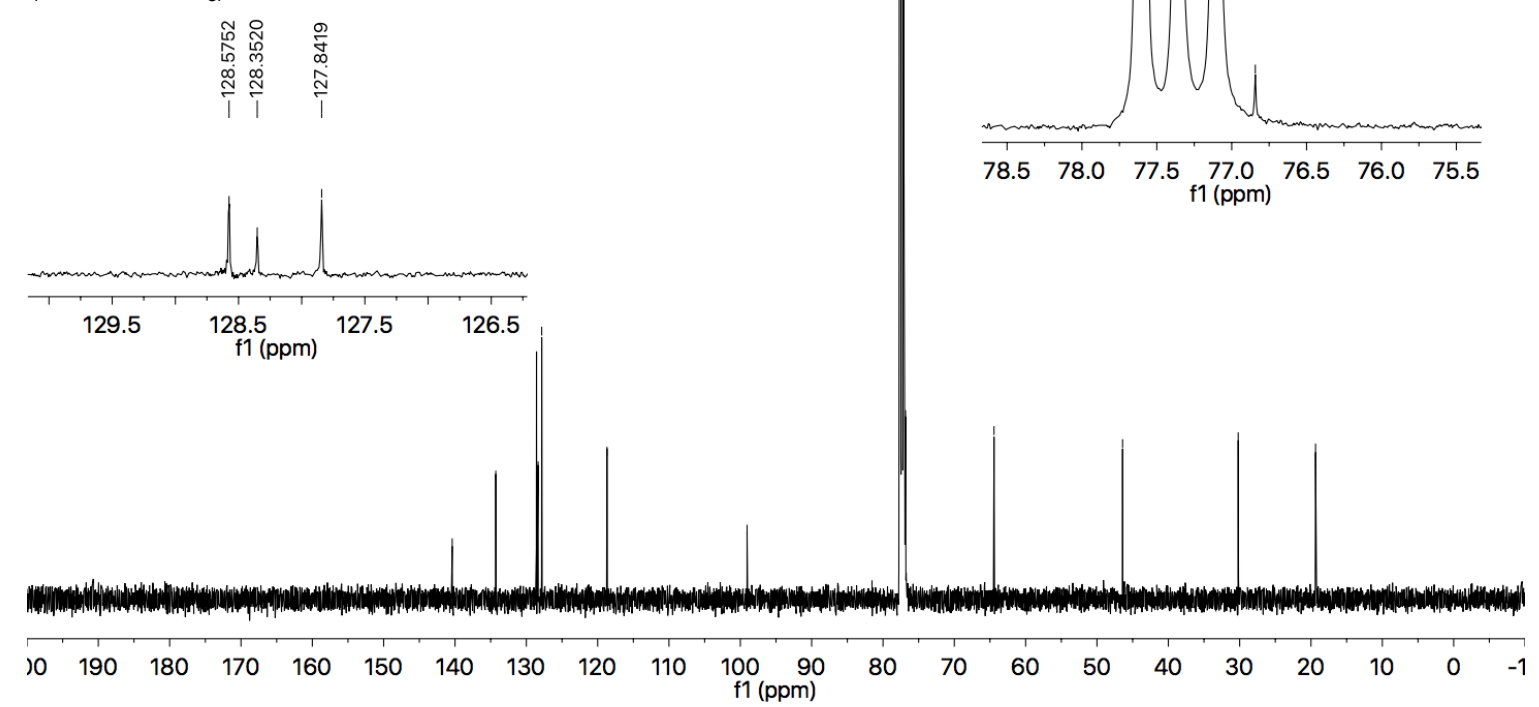

